\title{
Parametric Instabilities in Laser/Matter Interaction: From Noise Levels to Relativistic Regimes
}

\author{
H. A. Baldis \\ C. Labaune \\ W. L. Kruer
}

February 11, 1999

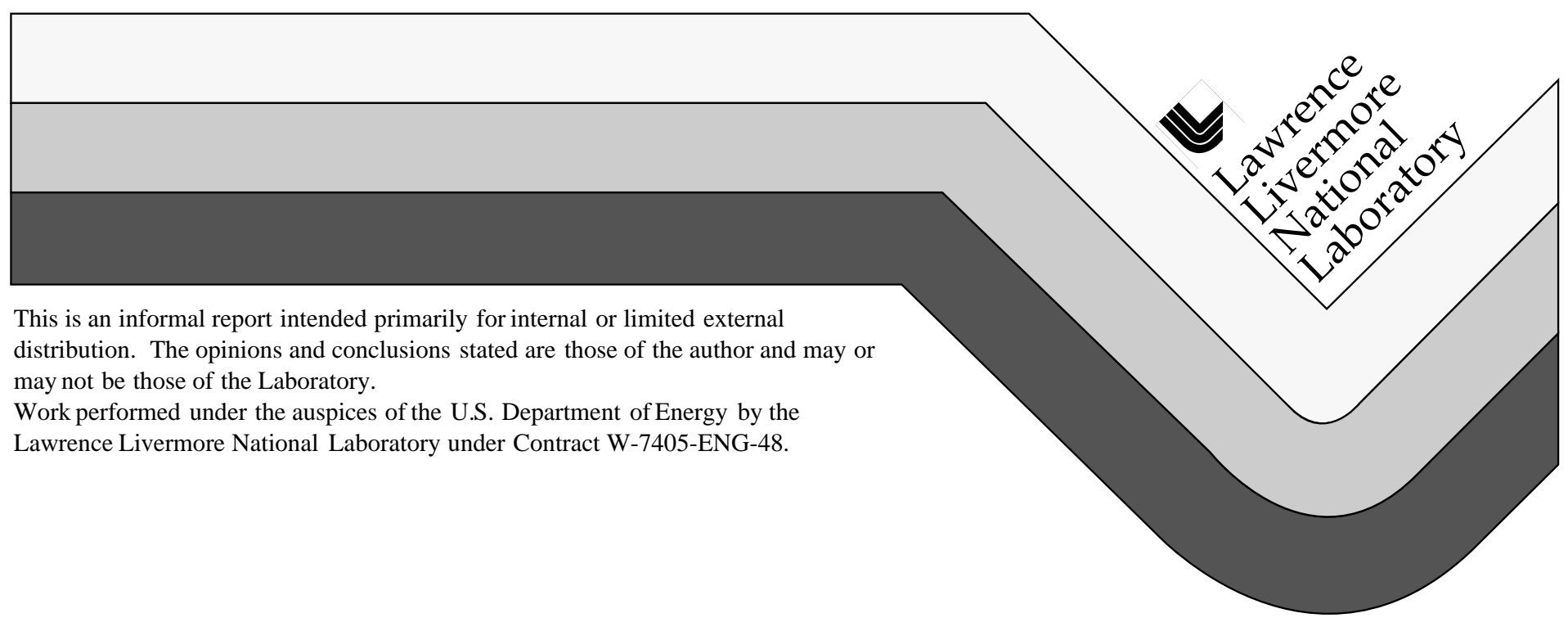




\section{DISCLAIMER}

This document was prepared as an account of work sponsored by an agency of the United States Government. Neither the United States Government nor the University of California nor any of their employees, makes any warranty, express or implied, or assumes any legal liability or responsibility for the accuracy, completeness, or usefulness of any information, apparatus, product, or process disclosed, or represents that its use would not infringe privately owned rights. Reference herein to any specific commercial product, process, or service by trade name, trademark, manufacturer, or otherwise, does not necessarily constitute or imply its endorsement, recommendation, or favoring by the United States Government or the University of California. The views and opinions of authors expressed herein do not necessarily state or reflect those of the United States Government or the University of California, and shall not be used for advertising or product endorsement purposes.

This report has been reproduced directly from the best available copy.

Available to DOE and DOE contractors from the Office of Scientific and Technical Information P.O. Box 62, Oak Ridge, TN 37831

Prices available from (423) 576-8401

Available to the public from the National Technical Information Service

U.S. Department of Commerce 5285 Port Royal Rd. Springfield, VA 22161 
UCRL-ID-133227

February 11, 1999

\section{Final report LDRD 96-ERI-011}

\section{Parametric Instabilities in Laser/Matter Interaction: From Noise Levels to Relativistic Regimes}

\section{H.A. Baldis, C. Labaune, and W.L. Kruer}

The purpose of this LDRD was the study of parametric instabilities on a laserproduced plasma, addressing crucial issues affecting the coupling between the laser and the plasma. We have made very good progress during these three years, in advancing our understanding in many different fronts. Progress was made in both theoretical and experimental areas.

The coupling of high-power laser light to a plasma through scattering instabilities is still one of the most complex processes in laser-plasma interaction physics. In spite of the relevance of these parametric processes to inertial confinement fusion (ICF) and all other situations where a high-power laser beam couples to a plasma, many aspects of the interaction remain unexplained, even after many years of intensive experimental and theoretical efforts. Important instabilities under study are stimulated Brillouin scattering (SBS), stimulated Raman scattering (SRS), and the Langmuir decay instability (LDI). The study of these instabilities is further complicated by the competition and interplay between them, and, in the case of ICF, by the presence of multiple overlapping interaction beams. 
Stimulated Brillouin scattering consists of the decay of the incident electromagnetic (EM) wave into a scattered EM wave and an ion acoustic wave (IAW). Similarly, SRS consists of the decay of the incident EM wave into a scattered EM wave and an electron plasma wave (EPW). Langmuir decay instability is the further decay of an EPW into a secondary EPW and an IAW.

The principal areas of research covered during this three-year period were the following:
a)
Modeling of Parametric Instabilities in Speckles
b)
Langmuir Decay Instability
c)
Non Maxwellian Plasmas
d)
Multiple Interaction Beams
e)
SBS from Speckle Distributions

\section{Modeling of Parametric Instabilities in Speckles}

The theory of parametric instabilities in randomized laser beams is based on the idea that a laser beam focused through a random-phase plate (RPP) in a plasma produces a statistical interference pattern that consists of many randomly distributed local maxima of intensity. In each maximum, called a "speckle", the laser field drives the parametric instabilities, each having a different reflectivity, depending on the laser power trapped in the particular beamlet. However, because of the small size and random locations of the speckles, experimental observations represent averaged data, which are the sum of emissions from many independent speckles integrated over the resolution time of the instruments. We applied this modeling 
to an experiment where the back-scattered light due to stimulated Brillouin scattering was resolved in space, across the laser's focal spot. In the experiment, we observed that more than $50 \%$ of the scattered light was generated in only a few localized speckles. This implies that the ratio of the local reflectivity from a speckle to the average one (if space resolution were ignored) was over 100 - a significant discrepancy if one is to compare the average reflectivity to existing theories on parametric instabilities.

\section{Langmuir Decay Instability}

The Langmuir Decay Instability (LDI) is of importance as a potential saturation mechanism for SRS. We studied Langmuir waves experimentally using Thomson scattering, in a collaborative effort with the laser facility at Ecole Polytechnique, France. Using Thomson scattering of a short wavelength probe beam, two EPW's with the same frequency and propagating in opposite directions were simultaneously observed. The primary EPW's were identified as an LDI product. Other possible mechanisms of production of the secondary EPW's were analyzed and ruled out. This experiment demonstrated that (1) even small density fluctuations associated with Langmuir waves can produce the Langmuir decay instability and (2) the decay of the Langmuir waves should be included in the modeling of SRS, even in the case of low reflectivities. 
The study of SBS and SRS is further complicated by the interplay between the instabilities and/or the decayed waves. Their growth in the presence of waves (either IAW or EPW) associated with another instability is an important element in any attempt to explain their behavior. In experimental conditions similar to the study of LDI, an anticorrelation between the levels of IAW's and EPW's has been observed by changing the initial conditions for interaction. Inhibition of SBS by SRS can explain the observed delay for the Raman growth. The spatial location of SBS can be explained partly by pump depletion inside the laser hot spots (speckles) which contribute the most to SBS, and partly by the overall intensity distribution along the laser axis. The interplay between these instabilities has been an experimental fact for over 10 years, and their understanding is a crucial element to determine the laser coupling and laser light distribution in plasmas created inside cavities such as hohlrams.

\section{Non Maxwellian Plasmas}

Non-Maxwellian electron velocity distribution functions exist in plasmas where there are sources of energy to sustain the plasma state away from the usual Maxwellian equilibrium. Whether it be nonlinear, many-wave interactions (such as described by quasi-linear theory), turbulence, a high-power laser heating a high- $Z$ plasma, or nonlocal heat transport in laser-produced plasmas due to steep temperature gradients - non-Maxwellian velocity distributions have long been identified for conditions that are readily accessible in experiments today. When the laser intensity is sufficiently high, electron-ion heating takes place fast enough that electron-electron collisions cannot equilibrate the distribution, giving rise to these 
novel states. Through this project, we developed the first systematic calculations of the solutions of the plasma-dispersion relation, taking into account electron velocity distributions that can be different from Maxwellian.

\section{Multiple Interaction Beams}

An important component of our research was the modification of the instabilities in the presence of multiple interaction beams. Enhanced forwardstimulated Brillouin scattering (SBS) has been theoretically predicted both in the case of crossing beams with different frequencies in homogeneous plasmas, and in the case of beams with same frequency in presence of plasma flow. This can produce energy transfer from one beam to the other or a redistribution of the laser energy along the forward direction.

We reported the first observation and spectral analysis of enhanced forwardscattered light of a laser beam in the presence of a second, identical, laser beam, crossing the first one at $22,5^{\circ}$. Results obtained with parallel and crossed polarization for the two beams demonstrate that the coupling between the beams must happen through the ion acoustic waves. Enhanced forward scattering is likely due to enhanced forward stimulated Brillouin scattering through same type of resonant process as what has been observed theoretically for symmetric modes, or experimentally for waves propagating along the bisector of two beams. Linear theory predicts no growth when the two laser beams have the same frequency, but because of the small angle between the two beams $\left(22,5^{\circ}\right)$, the frequency of ion acoustic waves involved in this process is very small compared to the laser frequency. The resonance conditions should be easily satisfied if one includes all possible 
modifications of laser frequencies through hydrodynamic effects, density evolution, local heating, or modifications of ion acoustic frequency through Doppler effect in the flowing plasma. The large width of the red-shifted component also indicates that a broad spectrum of ion acoustic waves can contribute to the coupling. If SBS is in the strongly coupled regime, nonresonant ion modes could be driven by the laser beam, with the frequency of these modes depending on the laser intensity.

\section{SBS from Speckle Distributions}

The localization of SBS was studied in a plasma irradiated using random phase plates (RPP) to produce a statistically predictable intensity distribution in the focal spot. We presented the first experimental evidence of the localization of SBS emission, in a plane perpendicular to the laser beam axis, demonstrating that only few small interaction regions contribute to the total SBS emission. SBS emission from these small spots has been evaluated leading to local reflectivity much higher than the average SBS reflectivity, by a factor $\sim 100$. These observations are consistent with the recent concept of convective amplification of SBS in randomly distributed speckles as produced by the RPP. This new approach is important as it can explain the moderate observed average SBS reflectivity, because of the limited number of SBS-active speckles, and pump depletion within these speckles. This statistical theory has been successful in explaining a number of different physical features of SBS in a recent experiment. The observation of localized regions of SBS emission observed in this experiment demonstrates the importance of the microscopic description of SBS. 


\section{Refereed Journal Publications}

The following refereed journal articles were published as part of the work covered under this LDRD.

Labaune, C., H. A. Baldis, N. Renard, E. Schifano, A. Michard, “Interplay between ion acoustic waves and electron plasma waves associated with stimulated Brillouin and Raman scattering," Phys. Plasmas, 4, 423, (1997) UCRL-JC-127429.

Baldis,H. A., and C. Labaune, "Interplay between parametric instabilities in the context of inertial confinement fusion," Plasma Physics and Controlled Fusion, 39, A51, (1997) UCRL-UR-121871.

Labaune, C., H. A. Baldis, V. T. Tikhonchuk, “Interpretation of stimulated Brillouin scattering measurements based on the use of random phase plates," Euro Phys. Lett., 38, 31 (1997) , UCRL-UR-121872.

Baldis, H. A., C. Labaune, J. D. Moody, T. Talinaud, V. T. Tikhonchuk, "Localization of stimulated Brillouin scattering in random phase plate speckles," Phys. Rev. Lett., 80, 1900-1903, (1998) UCRL-JC-128618.

Labaune, C., H. A. Baldis, B. S. Bauer, V. T. Tikhonchuk, G. Laval, “Timeresolved measurements of secondary Langmuir waves produced by the Langmuir decay instability," Phys. Plasmas, 5, 234-242, (1998) UCRL-JC-127432.

Cohen, B. I., B. F. Lasinski, A. B. Langdon, E. A. Williams, H. A. Baldis, C. Labaune, "Suppression of stimulated Brillouin scattering by seeded ion wave mode coupling," Phys. Plasmas, 5, 3402-3407, (1998) UCRL-JC-129490. 


\begin{abstract}
Labaune, C., H. A. Baldis, B. S. Bauer, E. Schifano, A. Michard, “Effect of crossed beams irradiation on Parametric Instabilities," Particle and Laser Beams, in press, (1999) UCRL-JC-130642.

Rozmus, W., S. Glenzer, K. Estabrook, H. A. Baldis, B. MacGowan, “Modelling of Thomson scattering spectra in high-Z, laser produced plasmas," Astrophysics. J., in press, (1999) UCRL-JC-130646.
\end{abstract}

This work was partially supported under the auspices of the U.S. Department of Energy by the Lawrence Livermore National Laboratory under contract No. W-7405-ENG-48 through the Institute for Laser Science and Applications. 

associated with stimulated Brillouin and Raman scattering

\author{
C. Labaune, H. A. Baldis, ${ }^{a)}$ N. Renard, E. Schifano, and A. Michard \\ Laboratoire pour l'Utilisation des Lasers Intenses (LULI), Centre National de la Recherche Scientifique, \\ Ecole Polytechnique, 91128 Palaiseau Cedex, France
}

(Received 3 June 1996; accepted 31 October 1996)

\begin{abstract}
Direct evidence is presented of the temporal and spatial interplay between ion acoustic waves (IAWs) associated with stimulated Brillouin scattering (SBS) and electron plasma waves (EPWs) associated with stimulated Raman scattering (SRS) in conditions of interest to inertial confinement fusion (ICF). The two types of waves grow over a limited region in the front part of the plasma, but at different times. Inhibition of the EPWs by the IAWs has been observed in the early part of the laser pulse, and this inhibition can be strengthened by increasing the level of the IAWs. (C) 1997 American Institute of Physics. [S1070-664X(97)01702-3]
\end{abstract}

\section{INTRODUCTION}

Stimulated Brillouin scattering (SBS) and stimulated Raman scattering (SRS) are among the most important parametric instabilities in laser-produced plasmas. ${ }^{1,2}$ SBS and SRS correspond to the decay of the incident electromagnetic (EM) wave into a scattered EM wave and an ion acoustic wave (IAW), or an electron-plasma wave (EPW), respectively. Apart from the intrinsic interest of the wave coupling mechanisms, these two instabilities have important implications in inertial confinement fusion (ICF) due to the possible large conversion of laser energy into scattered light affecting ICF drive efficiency and symmetry. ${ }^{3}$ The study of parametric instabilities driven in laser-produced plasmas offers the possibility to study not only the parametric process itself, but also the complex interaction between plasma waves in hightemperature high-density plasmas. Waves driven by parametric instabilities interact among themselves, modifying in turn the behavior of the instabilities. It has become clear in recent years that the interaction between different plasma waves yields an interplay between the parametric instabilities that obscures the characterization and understanding of the parametric process itself. Although the interplay between the daughter waves associated with the instabilities was observed more than ten years ago, ${ }^{4-6}$ little effort has been made towards the characterization and experimental study of this important phenomenon. A vital aspect of the evolution to the instabilities is their growth in the presence of waves (either IAW or EPW) associated with another instability, or their saturation associated with secondary decays, such as the parametric decay of the EPW from SRS. ${ }^{7}$ The study of parametric instabilities under these conditions is the extreme counterpart to similar studies in microwave pumped experiments in the laboratory, and in the ionosphere, using much longer hf decametric waves as a pump.

Considerable theoretical work has addressed the understanding of the SRS growth in the presence of ion acoustic waves from SBS. ${ }^{8}$ Numerical simulations have indicated the weakening of SRS by the presence of SBS, when ion fluc-

a) Also at the Institute for Laser and Plasma Sciences (ILSA), Lawrence Livermore National Laboratory, Livermore, California 94550. tuations are included in the calculation of the SRS growth. One of the possible explanations for this is the spoiling of the phase-matching requirement for SRS by the ion waves. The first experimental observation of competition between SRS and SBS was reported by Walsh et al., ${ }^{4}$ who observed a strong correlation between the quenching of SRS plasma waves and the initiation of SBS ion waves. In the same experiment, seeding SBS with a small amount of stimulated backscattered light ${ }^{5}$ caused the SRS emission to be completely eliminated. This experiment was performed with a much longer laser wavelength $(\lambda=10.6 \mu \mathrm{m})$ and with a much higher $I \lambda^{2}\left(\sim 10^{16} \mathrm{~W} \mu \mathrm{m}^{2} / \mathrm{cm}^{2}\right)$ than we will be discussing here ( $I$ is the laser intensity per unit area). These parameters placed the instabilities in the saturated regime for which modeling is difficult, and the low plasma temperature does not fit ICF plasmas well. A second experiment also observed an anticorrelation between SBS and SRS using one arm of Nova ${ }^{9}$ to form the plasma and to pump the instabilities. ${ }^{6}$ In this experiment the diagnostics were based on the scattered electromagnetic waves, which cannot permit as direct an observation of the instabilities as plasma waves.

In this paper, we present direct evidence of the temporal and spatial interplay between IAWs associated with SBS, and EPWs associated with SRS. Direct observation of these waves has been done using Thomson scattering of a shortwavelength probe beam. The two types of waves grow in the same region of plasma, but at different times. The EPWs start to grow only when the IAWs have disappeared. The level of the density fluctuations associated with the EPWs is further reduced when the IAWs level is enhanced by modifying the initial noise level from which they grow. The appearance of the waves at the beginning of the interaction pulse is opposite to what was observed in the $\mathrm{CO}_{2}$ experiment, which underlines the importance of the initial conditions on the instabilities growth and dominance. The important point is that these new data demonstrate the inhibition of Raman by Brillouin in a different regime compared to the previous experiments. The results have been produced by the interaction between a laser beam with a well-characterized laser intensity distribution obtained with a random phase plate (RPP) and a preformed plasma. The intensity of the interaction beam was intentionally kept low in order to pro- 


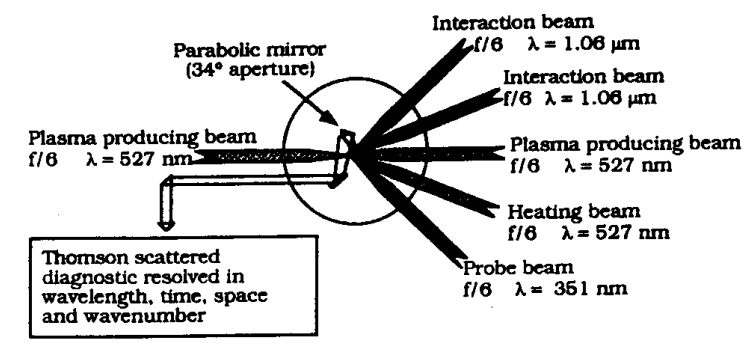

FIG. 1. Experimental arrangement. All laser beams and Thomson scattering collecting optics are in the same plane.

duce SBS and SRS below saturation, and to be able to apply the theory of parametric instabilities in statistically distributed independent speckles. ${ }^{10,11}$ One has to be careful with the interpretation of saturation: at the intensities used in this experiment, the instabilities are below saturation of the overall beam reflectivity, that is to say, the relatively small observed reflectivities will increase with increasing laser intensity. This does not imply that saturation does not occur inside the high-intensity speckles. This will be discussed further in Sec. IV.

\section{EXPERIMENTAL ARRANGEMENT}

The results presented here are part of the program at the Laboratoire pour l'Utilisation des Lasers Intenses (LULI) to study the physics of parametric instabilities in the context of ICF, using collective Thomson scattering as the key diagnostic. ${ }^{12}$ The experiments have been performed using the six-beam laser facility, to produce the interaction between a $1.053 \mu \mathrm{m}$ pump and a well-characterized preformed plasma. The absolute correlation in time and space of the waves associated with the instabilities has been made possible by a new technique ${ }^{13}$ developed for these studies, which permits us to multiplex a conventional streak camera, providing simultaneous recordings of the temporal evolution of the Thomson scattered light from the two types of waves on the same detector.

The laser beam configuration and experimental arrangement is shown in Fig. 1. The 600 ps full-width halfmaximum (FWHM) Gaussian beams were all in the same plane and arrived at the target at different times: two $0.53 \mu \mathrm{m}$ plasma-producing beams at $t=0$, a $0.53 \mu \mathrm{m}$ plasma heating beam at $t=1.2 \mathrm{~ns}$, the $0.35 \mu \mathrm{m}$ Thomson scattering probe beam at $t=1.48 \mathrm{~ns}$, and the $1.05 \mu \mathrm{m}$ interaction beam at $t=1.72 \mathrm{~ns}$ (timing refers to the peak of the pulse). Random phase plates (RPP) ${ }^{13}$ were used on the beams producing and heating the plasma to obtain plasmas as uniform and reproducible as possible. The interaction beam was focused with an $f / 6$ lens through a RPP of $2 \mathrm{~mm}$ square elements. The combination of the focusing lens and the RPP produces an Airy pattern central maximum with $65 \%$ of the total laser energy in a spot diameter of $320 \mu \mathrm{m}$. Within this spot, the maximum average intensity is $10^{14} \mathrm{~W} / \mathrm{cm}^{2}$, for an energy of $100 \mathrm{~J}$. The mean FWHM di- ameter of the speckles is $\sim 6 \mu \mathrm{m}$ and the mean length is $\sim 300 \mu \mathrm{m}$. The targets were mass-limited free-standing $\mathrm{CH}$ foils of $450 \mu \mathrm{m}$ diam and $1.5 \mu \mathrm{m}$ thickness.

The geometry for Thomson scattering was chosen to observe the scattered light that corresponds to IAWs associated with backscattered SBS and EPWs associated with backscattered SRS. The Thomson scattering probe was focused with a combination of a lens and a RPP with elongated elements, to form a focal region $100 \mu \mathrm{m}$ by $1 \mathrm{~mm}$ along the axis of the interaction beam, thus allowing us to image the location of the waves over the whole interaction length. The Thomson scattered light was collected with a parabolic mirror and imaged onto two different diagnostics by secondary spherical mirrors. Since the EPWs and IAWs of interest have different wave numbers, they were collected by different areas on the parabolic mirror. Masks were used as required, to select the waves of interest at different stages during the experiment. A double image of the plasma was formed at the entrance slits of a streak camera; separate filtration and attenuation in front of each slit permitted us to select the range of frequencies (IAW or EPW), and to adjust the level of the signals to fit within the dynamic range of the camera. This technique provides an absolute correlation in space and time between the two instabilities. A separate spectrograph-streak camera combination was used to obtain the time-resolved spectra of the waves.

The plasma was well characterized over many experimental campaigns. ${ }^{15}$ The electron density at the peak of the plasma profile evolved from $0.25 n_{c}$ to $0.08 n_{c}$, from the beginning to the end of the interaction beam (where $n_{c}=1.1 \times 10^{21} \mathrm{~cm}^{-3}$ is the critical electron density for $\lambda_{0}=1.05 \mu \mathrm{m}$ light). The density profile had a quasi-inverseparabolic form along the interaction axis, with a FWHM of $1 \mathrm{~mm}$. The electron temperature $\left(T_{e}\right)$ of the plasma, obtained by Thomson scattering in the absence of the interaction beam, was between 0.4 and $0.5 \mathrm{keV}$.

\section{RESULTS ON THE INTERPLAY}

Figure 2 shows the time-resolved location of IAWs and EPWs along the axis of the interaction beam. The scattered signals are related to the density fluctuations $(\delta n / n)_{\text {LAW,EPW }}$ associated with the IAWs and EPWs corresponding to backscattered SBS and SRS, respectively. If the scattering takes place from coherent plasma waves, the scattered power levels are proportional to the square of $(\delta n / n)_{\text {LAW,EPW }}$. Time is referred to the plasma-forming laser pulses, the initial position of the target was at $z=0$, and the interaction beam arrived from the right. Absolute timing and location of the signals were provided by the early plasma emission during the preforming pulses. For both backscatter instabilities the growth is limited to a relatively small region, in the front part of the plasma relative to the interaction beam. The interaction region is smaller than the scale length of the plasma and is not limited by the length of the line focus of the Thomson scattering probe. The interaction region for the IAWs shows, as a function of time, a drift toward the laser side; the EPWs, on the other hand, drift toward the center of the plasma.

Figure 3 shows the time-resolved spectra of IAWs and 




FIG. 2. Location of Thomson scattered light from IAWs and EPWs, associated with backscattered SBS and SRS, respectively, as a function of time, and recorded on the same streak camera. The Thomson scattered intensity is proportional to the square of the levels of the density fluctuations associated with the plasma waves. Position $z=0$ corresponds to the summit of the plasma. The interaction beam arrives from the right side. The difference between contours corresponds to a factor of 2 in intensity.

EPWs obtained with the second Thomson scattering diagnostic, using a low dispersion diffraction grating. These spectra correspond to plasma waves from a well-defined region of plasma, located $300 \mu \mathrm{m}$ from the summit toward the laser. The wave number of these waves was set by masks that limited the light collection to an aperture of $5^{\circ}$. A time reference was given by the plasma emission during the preforming pulses. This diagnostic provides the frequencies and wave numbers of the observed plasma waves, thus confirming their connection with backscattered Brillouin and Raman instabilities. By using three slits on the streak camera, good agreement with the first diagnostic was obtained on the spatial and on the temporal evolution of the plasma waves.

An interesting result is the temporal interplay between the two instabilities that is observed on the two complemen-

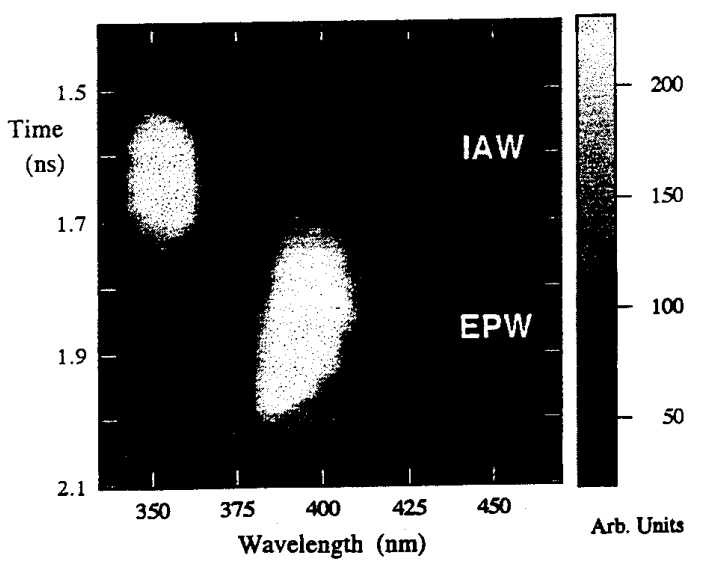

FIG. 3. Time-resolved spectra of Thomson scattered light from IAWs and EPWs.

Phys. Plasmas, Vol. 4, No. 2, February 1997 tary diagnostics. The onset of the IAWs takes place at the very beginning of the interaction pulse; the emission lasts around $300 \mathrm{ps}$ and is maximum $\sim 100$ ps before the peak of the interaction pulse. Simultaneously with the disappearance of the IAWs, one observes a rapid growth in the level of fluctuations associated with EPWs. The EPWs are observed only after the disappearance of the IAWs and the duration of the EPWs signal is around 300 ps. Further to the temporal interplay between IAWs and EPWs, we observe that, within $100 \mu \mathrm{m}$ (which represents shot to shot fluctuations), the EPWs are located in the same region of plasma where the IAWs just disappeared. This interplay between the two instabilities had been observed in all laser shots, and under various plasma conditions.

\section{DISCUSSION}

It will be useful to compare the present results with modeling of the instabilities, taking into account the laser intensity distribution produced by the random phase plate. The use of a random phase plate on the interaction beam has the advantage of producing a large number of statistically independent speckles in the interacting volume so that the standard statistical distribution over their intensities can be calculated. ${ }^{10}$ The theory of convective amplification of SBS in an inhomogeneous plasma, ${ }^{16,17}$ including diffraction and inhomogeneity of the flow velocity, has been applied to an individual speckle to calculate the SBS reflectivity and density fluctuations associated with the IAWs as a function of space and time. However, because of the small size and random location of speckles, experimental observations represent average data, which are the sum of emissions from many independent speckles integrated over the instrumental space and time resolution. The comparison between experiment and modeling is then based on the averaging of the previous quantities over the distribution of speckles. For the purpose of this paper, only results from the modeling will be discussed, leaving more details on the calculation and description of the modeling to Ref. 18.

The modeling predicts the same temporal evolution of SBS as the one that has been observed. The temporal increase of the SBS reflectivity follows the laser intensity, while the drop of the SBS gain is mainly due to the reduction of the electron density due to plasma expansion. Using the convective Rosenbluth gain ${ }^{19}$ and the plasma parameters, the average SBS gain (= the SBS gain calculated for average laser intensity) is

$$
\langle G\rangle \approx 0.046 I_{14}(t) \lambda_{0}\left(L_{u} / T_{e}\right)\left\{n_{e}(t) /\left[n_{c}-n_{e}(t)\right]\right\},
$$

where $I_{14}$ is the laser intensity in units of $10^{14} \mathrm{~W} / \mathrm{cm}^{2}, n_{e}$ is the electron density, $T_{e}$ is the electron temperature in $\mathrm{keV}$, and $L_{u}=c_{s}(d u / d z)^{-1}$ is the velocity gradient scale length. The maximum average SBS gain in this experiment is about 3 , and the model predicts that the major contribution to the averaged reflectivity comes from speckles with an intensity around four times above the average laser intensity, corresponding to local gains of 10-12. Such speckles are in the regime of strong pump depletion, which produces a shift of the maximum of density perturbations toward the laser as 


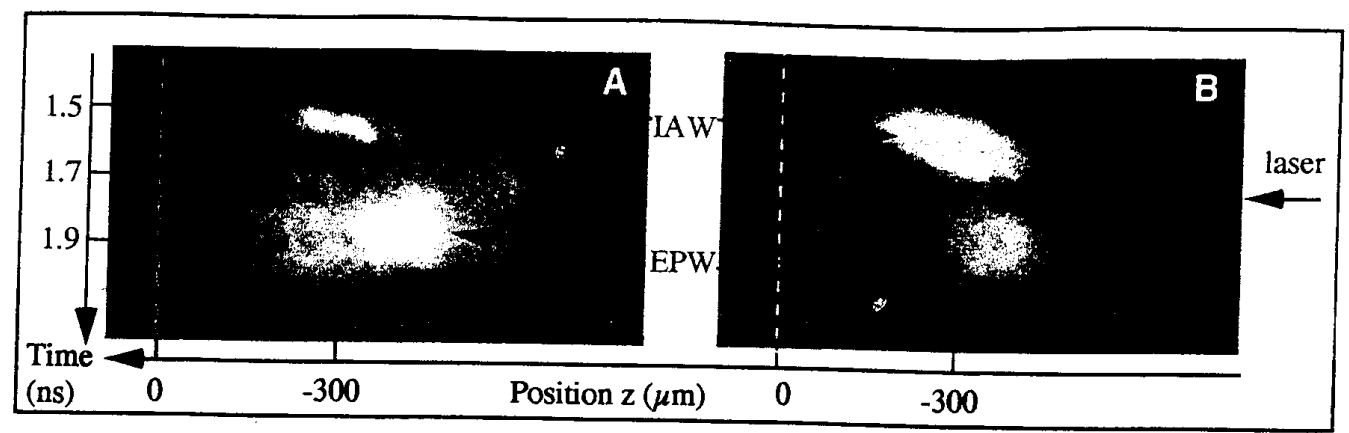

FIG. 4. Temporal evolution of the intensity of the Thomson scattered light from IAWs and EPWs. Frame B corresponds to enhanced initial noise level from which the LAWs grow compared to frame A. Same filtration has been used, in front of the streak camera, for these two shots.

they follow the intensity distribution. Combining this effect with the overall intensity distribution along the laser axis, including inverse bremsstrahlung absorption, the modeling predicts that the maximum of average density fluctuations associated with the LAWs is shifted by around $250-300 \mu \mathrm{m}$, in agreement with the observed location of the IAWs.

Contrary to SBS, the behavior of SRS cannot be predicted by modeling of the isolated instability. The main point is that SRS should start earlier in the laser pulse than what was observed, as is clear from the experiment that EPWs start only after the disappearance of IAWs. We assume that this inhibition of Raman early in the interaction pulse is due to the ion fluctuations associated with SBS, a phenomena previously observed in numerical simulations and in theoretical treatment of the instabilities, as indicated earlier in this paper. ${ }^{8}$ To test this hypothesis, a new set of experiments was made, where the initial noise level from which SBS grows was purposely enhanced by seeding it with initial IAWs having matching characteristics to the SBS ion waves. ${ }^{20}$ Measurements of space- and time-resolved density fluctuations of the two types of waves are presented in Fig. 4, where frames $A$ and $B$ correspond to the standard shot, and to the shot with enhanced IAWs noise level, respectively. We definitely observe anticorrelation in the change of the levels of the IAWs and EPWs, which demonstrate that an increased level of IAWs produces a reduction of the level of EPWs.

\section{SUMMARY AND CONCLUSIONS}

We have presented direct evidence of temporal and spatial interplay between IAWs associated with SBS and EPWs associated with SRS. Anticorrelation between the levels of IAWs and EPWs has been observed by changing the initial conditions for interaction. Inhibition of Raman by Brillouin can explain the observed delay for the Raman growth. The spatial location of SBS can be explained partly by pump depletion inside the speckles, which contribute the most to SBS, and partly by the overall intensity distribution along the laser axis. The same argument can be used to explain the SRS location, as it follows the resultant intensity distribution. This could also explain the drift of EPWs toward the summit of the plasma as a function of time: pump depletion produced by active SBS disappears when SBS drops, and the peak intensity comes back to the center of the plasma. The results presented in this paper show the importance of taking into account the interplay between instabilities to successfully characterize and understand the behavior of parametric instabilities in laser plasmas. This is particularly true in hohlraums plasmas, where a large number of laser beams overlap. The problem of interplay between SRS and SBS is further complicated by the interplay between different modes of SBS pumped by different interaction beams, as discussed in Ref. 20.

\section{ACKNOWLEDGMENTS}

The authors acknowledge useful discussions with V. T. Tikhonchuk, the participation of B. Quesnel to the experiment, the target fabrication of $\mathrm{H}$. Timsit and $\mathrm{C}$. Coulaud, and the laser group of LULI, who made this experiment possible.

Part of this work was performed under the auspices of the U.S. Department of Energy by the Lawrence Livermore National Laboratory under Contract No. W-7405-Eng-48.

${ }^{1}$ W. L. Kruer, The Physics of Laser Plasma Interactions (Addison-Wesley, Redwood City, CA, 1988).

${ }^{2}$ H. A. Baldis, E. M. Campbell, and W. L. Kruer, in Physics of Laser Plasma, edited by A. Rubenchik and W. Witkowski (North-Holland, Amsterdam, 1991).

${ }^{3}$ J. Lindl, Phys. Plasmas 2, 3933 (1995).

${ }^{4}$ C. J. Walsh, D. M. Villeneuve, and H. A. Baldis, Phys. Rev. Lett. 53, 1445 (1984).

${ }^{5}$ D. M. Villeneuve, H. A. Baldis, and J. Bernard, Phys. Rev. Lett. 59, 1585 (1987).

${ }^{6}$ H. A. Baldis, P. E. Young, R. P. Drake, W. L. Kruer, K. Estabrook, E. A Williams, and T. W. Johnson, Phys. Rev. Lett. 62, 2829 (1989).

${ }^{7}$ K. L. Baker, R. P. Drake, B. S. Bauer, K. G. Estabrook, A. M. Rubenchik, C. Labaune, H. A. Baldis, N. Renard, S. D. Baton, E. Schifano, A. Michard, W. Seka, and R. E. Bahr, Phys. Rev. Lett. 77, 67 (1996).

${ }^{8}$ P. K. Kaw, A. T. Lin, and J. M. Dawson, Phys. Fluids 16, 1967 (1973); W. Rozmus, A. A. Offenberger, and R. Fedosejevs, ibid. 26, 1071 (1983); H. C. Barr, and F. Chen, ibid. 30, 1180 (1987); G. Bonnaud, Laser Part. Beams 5, 101 (1987); M. C. Aldrich, B. Bezzerides, D. F. DuBois, and H. Rose, Comments Plasma Phys. Controlled Fusion 10, 1 (1986); H. A. Rose, D. F. DuBois, and B. Bezzerides, Phys. Rev. Lett. 58, 2547 (1987); W. Rozmus, R. P. Sharpa, J. C. Samson, and W. Tighe, Phys. Fluids 30, 2181 (1987).

${ }^{9} \mathrm{~J}$. Holzrichter, Nature 316, 309 (1985).

${ }^{10} \mathrm{H}$. A. Rose and D. F. DuBois, Phys. Fluids B 5, 590 (1993).

${ }^{11}$ H. A. Rose and D. F. DuBois, Phys. Rev. Lett. 72, 2883 (1994); H. A. Rose, Phys. Plasmas 2, 2216 (1995).

${ }^{12}$ C. Labaune, H. A. Baldis, N. Renard, E. Schifano, S. Baton, A. Michard, 
W. Seka, R. E. Bahr, B. Bauer, K. Baker, and K. Estabrook, Phys. Rev. Lett. 75, 248 (1995).

${ }^{13}$ H. A. Baldis and C. Labaune, Rev. Sci. Instrum. 67, 451 (1996).

${ }^{14}$ Y. Kato, K. Mima, N. Miyanaga, S. Arinaga, Y. Kitagawa, M. Nakatsuka and C. Yamanaka, Phys. Rev. Lett. 53, 1057 (1984)

${ }^{15}$ W. Seka, R. S. Craxton, C. Labaune, H. A. Baldis, N. Renard, E. Schifano, and A. Michard, Bull. Am. Phys. Soc. 40, 1777 (1995).
${ }^{16}$ L. M. Gorbunov and A. N. Polyanichev, Sov. Phys. JETP 47, 290 (1978).

${ }^{17}$ A. Ramani and C. E. Max, Phys. Fluids 26, 1079 (1983).

${ }^{18}$ V. T. Tikhonchuk, C. Labaune, and H. A. Baldis, Phys. Plasmas 3, 3777 (1996).

${ }^{19}$ M. N. Rosenbluth, Phys. Rev. Lett. 29, 565 (1972).

${ }^{20}$ H. A. Baldis, C. Labaune, E. Schifano, N. Renard, and A. Michard, Phys. 


\title{
Modelling of Thomson scattering spectra in high-Z, laser produced plasmas
}

\author{
W. Rozmus*, S. H. Glenzer, K. G. Estabrook, H. A. Baldis, B. J. MacGowan \\ Lawrence Livermore National Laboratory, Livermore, CA 94550.
}

(April 30, 1998)

\begin{abstract}
Theoretical calculations of a Thomson scattering cross section and dynamical form factors are presented for high-Z laser produced inhomogeneous plasmas. Relevance of these results to astrophysical plasmas is pointed out. Comparisons with recent experimental observations are discussed with emphasis on the effects of plasma inhomogeneity, ion-ion collisions and non-Maxwellian distribution functions.
\end{abstract}




\section{INTRODUCTION}

Thomson scattering (TS) is an important radiative process involving incoherent scattering of electromagnetic waves by free electrons in plasmas. TS has several applications in astrophysics. In stellar interiors scattering from free electrons is one of the basic processes contributing to the radiative opacity [1]. It has been shown recently that by properly accounting for the plasma response in the calculations of the TS cross-section one can reduce the solar opacity [2] and hence change predictions for the core parameters. This has been done by including broadening of the Langmuir wave resonance due to the Doppler effect and particle collisions.

With the advent of laser plasma interaction experiments in the regime of parameters of interest to astrophysics, TS becomes extremely important as the accurate plasma diagnostics. One of the main applications of the laboratory astrophysics is benchmarking of numerical codes used in modelling of astrophysical processes. This can be only accomplished with the help of reliable laboratory plasma diagnostic techniques such as the TS.

Since early days of laser plasma interaction physics, TS has been perfected as the diagnostic of enhanced levels of fluctuations due to parametric instabilities. Larger scattering powers from non-thermal fluctuations are easier to detect and many of these measurements have fundamental importance elucidating nonlinear saturation processes which are valid in the wide range of laser produced plasmas. Scattering from stable plasmas for the purpose of defining electron density, temperatures of electrons and ions, flow velocities, ionization levels, particle distribution functions and thermal transport has been also copducted. Several interesting experiments have characterized low temperature laser produced plasmas $[3,4]$, $x$-ray laser plasmas [5] and the inertial confinement fusion targets [6].

In this paper we discuss some theoretical results related to the calculations of the TS cross section in inhomogeneous plasmas and dynamical form factors which are valid under different physical conditons. Such theories and improved experimental techniques lead to further development of the TS as the most versatile and accurate diagnostic of laser produced 
plasmas.

In Section II we derive an expression for the TS cross section in inhomogeneous plasmas. Section III summarizes different results for the dynamical form factor: in collisionless inhomogeneous plasmas, plasmas with frequent ion-ion collisions and in plasmas described by the non-Maxwellian distribution functions. Conclusions and discussion are presented in Sec. IV.

\section{THOMSON SCATTERING FROM INHOMOGENEOUS PLASMAS}

Hot and dense plasmas produced by powerful lasers are almost always inhomogeneous. This is also a desirable feature in the laboratory astrophysics applications, where expanding corona or blow-off plasma is close to a "real" astrophysical conditions [cf. Ref. [7]]. Following the standard procedure (cf. e.g. [8]), we first derive the scattering cross section for the incoherent Thomson scattering in such plasmas. The incident pump is modelled by,

$$
\mathrm{E}_{0}(\mathbf{r}, t)=E_{0}(\mathbf{r}) \hat{\eta}_{0} \cos \left(\omega_{0} t-\mathbf{k}_{0} \cdot \mathbf{r}\right)
$$

We have assumed that within the scattering volume the probe is well approximated by the cylindrical laser beam with the prescribed polarization $\hat{\eta}_{0}$. The scattered radiation field is described by the vector potential $\mathbf{A}(\mathbf{r}, t)$ which at the large distance, $r$, from the plasma is approximated by the following expression,

$$
\mathbf{A}(\mathbf{r}, t)=\frac{1}{c r} \int_{V} d^{3} r^{\prime} \int d t^{\prime} \delta \mathbf{J}\left(\mathbf{r}^{\prime}, t^{\prime}\right) \delta\left(t^{\prime}-t+r / c-\hat{n} \cdot \mathbf{r}^{\prime} / c\right)
$$

where $\mathbf{r}^{\prime}$ varies within the scattering volume $V$ and the separation of scales, $r \gg r^{\prime}$, has been used in the approximate definition of the retarded time. The fluctuating nonlinear current $\delta \mathbf{J}$ is the result of a coupling between the Thomson probe (1) and the electron density fluctuations. From the solution to kinetic equation in the quasilinear approximation one obtains

$$
\delta \mathbf{J}(\mathbf{r}, t)=\frac{e^{2}}{m_{e}} \int \frac{d z}{2 \pi} \exp (-i z t) \frac{1}{z} \delta \mathbf{S}(\mathbf{r}, z)
$$


where the fluctuating source $\delta \mathrm{S}$ is the Laplace transformed product of the fluctuating electron density, $\delta N^{e}$, and the laser pump (1),

$$
\delta \mathbf{S}(\mathbf{r}, t)=\delta N^{e}(\mathbf{r}, t) \mathbf{E}_{0}(\mathbf{r}, t)
$$

In the derivation of Eq. (3) we have assumed that the plasma is almost transparent to the probe laser, i.e. $\omega_{0} \gg \omega_{p}$ and $\omega_{0} \gg \mathbf{k} \cdot \mathbf{v}$, where $\mathbf{k}$ is the wave vector of a collective plasma response and $\mathbf{v}$ is the characteristic particle velocity. The fluctuating current (3) provides a source of the radiation at the frequency $\omega_{1}$, which is close to the probe frequency $\omega_{0}$. A large value of $\omega_{1}$ simplifies description of a plasma response to scattered radiation and the propagation of a laser light inside the plasma. In the radiation zone, fields are approximated by the following expressions [9]

$$
\mathrm{B}_{1}=\frac{1}{c} \hat{n} \times \dot{\mathbf{A}}, \quad \mathbf{E}_{1}=\frac{1}{c} \hat{n} \times(\hat{n} \times \dot{\mathbf{A}}),
$$

where $\hat{n}$ is the unit vector along the direction of $\mathbf{r}$. Suppose that the instrument measures the scattered electric field along the direction of a unit vector $\hat{\eta}_{1}$. To calculate energy flux of the scattered radiation at the distance $r$ from the plasma we first derive the statistically averaged value of a Poynting vector

$$
\langle\mathcal{P}\rangle=\frac{c}{4 \pi}\left(\hat{\eta}_{0} \cdot \hat{\eta}_{1}\right)^{2} \frac{1}{c^{2}}\left\langle\dot{\mathbf{A}}^{2}(\mathbf{r}, t)\right\rangle
$$

Using formulas (2), (3) and (5) in Eq. (6) we find the following expression

$$
\begin{aligned}
\langle\mathcal{P}\rangle= & \frac{c}{4 \pi}\left(\hat{\eta}_{0} \cdot \hat{\eta}_{1}\right)^{2} \frac{1}{4 r^{2}} r_{0}^{2} \int_{V} d^{3} r^{\prime} \int_{V} d^{3} r_{1}^{\prime} \int \frac{d \omega}{2 \pi} E_{0}\left(\mathbf{r}_{1}^{\prime}\right) E_{0}\left(\mathbf{r}^{\prime}\right)\left\langle\delta N^{e}\left(\mathbf{r}^{\prime}\right) \delta N^{e}\left(\mathbf{r}_{1}^{\prime}\right)\right\rangle(\omega) \\
& \left\{\exp \left[-i \frac{\omega+\omega_{0}}{c} \hat{n} \cdot\left(\mathbf{r}^{\prime}-\mathbf{r}_{1}^{\prime}\right)+i \mathbf{k}_{0} \cdot\left(\mathbf{r}^{\prime}-\mathbf{r}_{1}^{\prime}\right)\right]+c . c .\right\}
\end{aligned}
$$

where $r_{0}=e^{2} / m_{e} c^{2}$. The symbol $\left\langle\delta N^{e}\left(\mathbf{r}^{\prime}\right) \delta N^{e}\left(\mathbf{r}_{1}^{\prime}\right)\right\rangle(\omega)$ defines Fourier transformed in time density correlation function. We have used the relation

$$
\left\langle\delta N^{e}(\omega) \delta N^{e}\left(\omega^{\prime}\right)\right\rangle=2 \pi\left\langle\delta N^{e} \delta N^{e}\right\rangle(\omega) \delta\left(\omega+\omega^{\prime}\right)
$$

which is valid in the stationary plasmas. 
Details of the derivation presented above are necessary to define limits of validity of the Thomson scattering cross-section in inhomogeneous plasmas. We will consider an example of the experimental data $[10,12]$ where the inhomogeneity of the plasma flow and density play an important role in the calculation of a cross-section. One can easily account for this effect if the spatial scale of density and flow variations is much longer than the correlation length of electron fluctuations. In stable plasmas the correlation length of a density correlation function $\left\langle\delta N^{e}\left(\mathbf{r}^{\prime}\right) \delta N^{e}\left(\mathbf{r}_{1}^{\prime}\right)\right\rangle$ is of the order of a Debye length, $\lambda_{D}$. This is a much shorter distance than the hydrodynamical scale of plasma parameter variations or the spatial variations of a probe intensity in the plasma. Exploring this separation of scales we introduce in Eq. (7) a Fourier transform with respect to a difference between spatial variable, $\mathbf{r}^{\prime}-\mathbf{r}_{1}^{\prime}$, and retain a long scale spatial variations of the time averaged probe energy flux $c E_{0}^{2}\left(\mathbf{r}^{\prime}\right) / 8 \pi$ and of the dynamical form factor $S\left(\mathbf{k}, \omega ; \mathbf{r}^{\prime}\right)=\left\langle\delta N^{e} \delta N^{e}\right\rangle(\mathbf{k}, \omega) / n_{0 e}$. The argument $\mathbf{r}^{\prime}$ in the dynamical form factor, $S$, corresponds to long scale variations described by the hydrodynamical variables. We have introduced $S(\mathbf{k}, \omega)$, which is calculated locally at different points in an inhomogeneous plasma. Thus, the expression for the scattered power, $P_{s}$, into a solid angle $d \Omega$ in the frequency range $d \omega$ reads

$$
P_{s}=\left(\hat{\eta}_{0} \cdot \hat{\eta}_{1}\right)^{2} r_{0}^{2} n_{0 e} \frac{d \Omega}{2 \pi} \int_{V} d^{3} r^{\prime} \frac{c E_{0}^{2}\left(\mathbf{r}^{\prime}\right)}{8 \pi} S\left(\mathbf{k}, \omega ; \mathbf{r}^{\prime}\right)
$$

where the wave-vector $\mathbf{k}$ and the frequency $\omega$ are related by the condition

$$
\mathbf{k}=\mathbf{k}_{0}-\frac{\omega_{0}-\omega}{c} \hat{n}
$$

The expression for the power scattered by electrons (8) at the frequency $\omega_{1}=\omega_{0}-\omega$ depends on the electron density, scattering volume $V$, and the dynamical form factor $S(\mathbf{k}, \omega)$ describing a plasma response. Expression (9) shows a relation between the direction of observation $\hat{n}$ and the wave-vector $\mathbf{k}$ which is probed in the plasma. For $k<k_{D}$ the main contributions to the scattering cross section are provided by the resonances at frequencies $\omega$ corresponding to the ion-acoustic and Langmuir fluctuations at the particular $k$. 


\section{DYNAMICAL FORM FACTOR}

The usefulness of the TS as the diagnostic technique is greatly enhanced when applied with the relevant theoretical expressions for the $S(\mathbf{k}, \omega)$. Equally important is a proper modelling of the TS cross-section in the radiation transport codes.

Consider three different expressions for a dynamical form factor which are applicable in plasmas: without collisions, with high ion-ion collisionality and described by the nonMaxwellian electron distribution function produced during an intense inverse bremsstrahlung heating. All three cases illustrate the potential of the TS as the plasma diagnostic, and are of relevance to the TS measurements from the high-Z, gold plasmas [10].

\section{A. Collisionless approximation}

In collisionless plasmas the power spectrum of density fluctuations in the frequency and wave vector space is a well known quantity (cf. e.g. [11]), which reads

$$
\begin{aligned}
S(\mathbf{k}, \omega) & =\frac{2 \pi}{k}\left|1-\frac{\chi^{e}(\mathbf{k}, \omega)}{\epsilon(\mathbf{k}, \omega)}\right|^{2} \bar{f}^{e}(v=\omega / k) \\
& +\left|\frac{\chi^{e}(\mathbf{k}, \omega)}{\epsilon(\mathbf{k}, \omega)}\right|^{2} \sum_{\beta(\neq e)} Z_{\beta}^{2} \frac{n_{0 \beta}}{n_{0 e}} \bar{f}^{\beta}(v=\omega / k),
\end{aligned}
$$

where $\bar{f}^{\beta}(v=\omega / k)$ stands for the one dimensional distribution function of a $\beta$-type particles evaluated at the velocity $\omega / k$. A dielectric response function

$$
\epsilon(\mathbf{k}, \omega)=1+\sum_{\boldsymbol{\beta}} \chi^{\beta}(\mathbf{k}, \omega)
$$

is defined by the susceptibility function,

$$
\chi^{\alpha}(\mathbf{k}, \omega)=\frac{\omega_{p \alpha}^{2}}{k^{2}} \int d^{3} v \frac{1}{\omega-\mathbf{k} \cdot \mathbf{v}+i \epsilon} \mathbf{k} \cdot \frac{\partial f^{\alpha}(\mathbf{v})}{\partial \mathbf{v}}
$$

Since the original derivation of an expression for the dynamical form factor (cf. Ref. [11] and references therein), Eq. (10) has been applied in the interpretation of numerous experiments. Here we would like to illustrate two features of this theory: $(i) S(\mathbf{k}, \omega)(10)$ can be calculated 
locally with the $f^{\alpha}$ given, for example, by the local Maxwellian in inhomogeneous plasmas with a scale separation between the correlation length and hydrodynamical gradients (as discussed in Sec. II); (ii) the most straightforward and reliable measurement of plasma parameters can be obtained from the simultaneous scattering spectra in frequency ranges corresponding to ion acoustic and Langmuir resonances.

Figure 1 shows $S(\mathbf{k}, \omega)(10)$ for the parameters of a gold homogeneous plasma $(Z=42$, $T_{e}=2 \mathrm{keV}, n_{e}=2.1 \times 10^{20} \mathrm{~cm}^{-3}$ ) plotted as the function of a scattered light wavelength for $\left(k \lambda_{D}\right)^{-1}=2.3$. The probe wavelength is $5266 \dot{A}$. From the separation of two ion acoustic peaks, Fig. 1, one can estimate the product $Z T_{e}$, which defines in high- $Z$ plasmas the value of an ion-acoustic frequency. The Langmuir wave resonance (Fig. 1) is then used to calculate elctron density, $n_{0 e}$, and temperature, $T_{e}$. This can be done with a reasonable accuracy because for the small value of $\left(k \lambda_{D}\right)^{-1}$, corresponding to strongly damped Langmuir fluctuations the overall shape of the $S(\mathbf{k}, \omega)$ is sensitive to both temperature and density variations. After finding $T_{e}$ one can improve an estimate of $Z$ by matching again the separation between two ion-acoustic peaks with a more accurate estimate of $T_{e}$. Figure 1 illustrates also the difficulties in practical realizaton of this straightforward procedure. They are related to very different sensitivities required in measurements of low and high frequency parts of the spectra both in terms of spectral resolutions and sensitivities of the instruments.

The scattering cross-section from Eq. (8) which is calculated using $S(\mathbf{k}, \omega)(10)$ and local Maxwellian distribution functions

$$
f^{\alpha}(\mathbf{v}, \mathbf{r})=\left(\frac{m_{\alpha}}{2 \pi T_{\alpha}(\mathbf{r})}\right)^{3 / 2} \exp \left[-\frac{m_{\alpha}\left(\mathbf{v}-\mathbf{u}_{\alpha}(\mathbf{r})\right)^{2}}{2 T_{\alpha}(\mathbf{r})}\right], \quad(\alpha=e, i),
$$

is shown in Fig. 2. Calculations of the spatial integral in Eq. (8) is simplified by the geometry of the experiment, where the direction of observation, $\hat{n}$, is approximately in the plane of $E_{0}(\mathbf{r})$ variations, i.e. in the transverse direction to $\mathbf{k}_{0}$. Figure 2 shows an ion-acoustic resonance which is Doppler shifted due to the flows, $\mathbf{u}_{\alpha}$ and exhibits small asymmetry between the peaks due to the drift of electrons with respect to ions, $u_{e} \neq u_{i}$. Note how much broader are the resonances as compared to simple theory in Fig. 1. This is almost entirely 
due to the inhomogeneity of the flows, which are approximated by the linear functions of position, $u_{\alpha}=u_{0 \alpha}\left(1+x / L_{u}\right)$, where $x$ is along the direction of the expanding plasma and $L_{u}=400 \mu \mathrm{m}$ is also in the very good agreement with hydrodynamical simulations.

\section{B. Frequent ion-ion collisions}

One of the characteristics of highly ionized plasmas is a small Landau damping of ion acoustic waves due to the large ratio, $Z T_{e} / T_{i}$. Small damping is responsible for sharpness of the ion acoustic resonances shown in Fig. 1. At the same time a large $Z$ value makes ion-ion collision frequency, which is proportional to $Z^{2}$, comparable to ion-acoustic frequency. Therefore, plasma response at the ion-acoustic resonance, described by $S(\mathbf{k}, \omega)$ should include collisional effects.

The formulation of a dynamical form factor which is valid in the entire regime of particle collisionality has been recently discussed in Ref. [13]. This theory is based on the nonlocal fluctuating hydrodynamical model. $S(\mathbf{k}, \omega)$ takes the following form in the limit of collisionless electrons and collisional ions,

$$
S(k, \omega)=\frac{4 k^{2}\left(c_{s}^{2}+v_{T_{i}}^{2}\right) \gamma_{a}}{\left(\omega^{2}-k^{2} v_{s}^{2}\right)^{2}+4 \omega^{2} \gamma_{a}^{2}},
$$

where we have introduced the definitions,

$$
v_{s}=\sqrt{c_{s}^{2}+\Gamma_{i} v_{i}^{2}}, \quad \text { and } \quad \Gamma_{i}=\frac{5}{3}+\frac{4}{3} \frac{\omega}{\nu_{i}} \operatorname{Im} \bar{\eta}=\frac{9 \omega^{4}+29.7 \omega^{2} \nu_{i}^{2}+11.7 \nu_{i}^{4}}{3\left(\omega^{4}+4.05 \omega^{2} \nu_{i}^{2}+2.33 \nu_{i}^{4}\right)}
$$

for the ion acoustic group velocity and ion specific heat ratio. The damping of an ion-acoustic wave, $\gamma_{a}$ involves electron Landau damping and the frequency dependent ion viscosity, $\tilde{\eta}$,

$$
\gamma_{a}=\sqrt{\frac{\pi}{8}} \frac{c_{s}}{v_{T e}} k v_{s}+\frac{2}{3} \frac{k^{2} v_{T i}^{2}}{\nu_{i}} \operatorname{Re} \tilde{\eta}
$$

where,

$$
\operatorname{Re} \tilde{\eta}=\frac{3}{2} k^{2} v_{T_{i}}^{2} \frac{\nu_{i}^{2}\left(1.49 \nu_{i}^{2}+0.80 \omega^{2}\right)}{\omega^{4}+4.05 \nu_{i}^{2} \omega^{2}+2.33 \nu_{i}^{4}}
$$


and $\nu_{i}=4 \sqrt{\pi} Z^{4} e^{4} n_{i} \Lambda_{i} / 3 \sqrt{m_{i}} T_{i}^{3 / 2}$ is the ion-ion collision frequency $\left(\Lambda_{i}\right.$ is a Coulomb logarithm). In spite of high values of $\nu_{i}$ in gold plasmas the effect of collisions on the shape of an ion-acoustic resonance is moderate. As expected Eq. (14) produces broadening of the resonance as compared to collisionless expression (10). However, as the values of $S(k, \omega)$ differ by only $10-30 \%$, this improvement has negligible effect on the interpretation of experimental results $[10,12]$. There, a $0.5 \dot{A}$ resolution of the spectrometer and an inhomogeneity of the plasma are far more important in determining the shape of the resonance.

\section{Super-Gaussian distribution function}

An important characteristic of a dense high- $Z$ plasma is a strong inverse bremsstrahlung (IB) absorption. Langdon [14] has shown that due to $\mathbb{B}$ the electron distribution function would take the form proportional to $\exp \left[-(v / u(t))^{\mu}\right], 2 \leq \mu \leq 5$, for the parameter $Z v_{0}^{2} / v_{e}^{2} \gg$ 1 , where $v_{0}$ is the oscillatory velocity of an electron in the electric field of the electromagnetic wave and $u(t)$ increases in time during IB plasma heating. Next, Matte et al. [15] introduced a stationary version of this distribution function

$$
f^{e}(v)=\frac{\mu}{4 \pi v_{e}^{3} \Gamma(3 / \mu)} \exp \left[-\left(\frac{v}{v_{*}}\right)^{\mu}\right]
$$

where $v_{*}^{2}=3 \Gamma(3 / \mu) v_{e}^{2} / \Gamma(5 / \mu)\left[v_{e}^{2}=(1 / 3) \int d^{3} v v^{2} f^{e}(v)\right]$ and the index $\mu$ is related to the laser intensity by the fitting expression

$$
\mu=2+3 /\left(1+1.66 / \alpha^{0.724}\right), \alpha=Z v_{0}^{2} / v_{e}^{2}
$$

In the experimental measurements $[10,12]$, which motivate our study, the parameter $\mu(19)$ corresponds approximately to $3 \leq \mu \leq 3.5$. The Thomson probe in these measurements has an intensity $I \approx 2.5 \times 10^{14} \mathrm{~W} / \mathrm{cm}^{2}$ and gives rise to $\mathrm{IB}$ heating.

Zheng et al [16] suggested that a Thomson scattering could be applied to verify theoretical predictions (18) for the electron distribution function. They pointed out that only simultaneous measurements of the scattered light in the frequency regimes corresponding to 
ion-acoustic and Langmuir wave resonances could provide necessary data to infer electron temperature and density in plasmas described by the non-Maxwellian distribution functions. These distribution functions change the ion-acoustic dispersion relation and therefore could complicate identification of the electron temperature. The Langmuir wave resonance for the parameters of the experiment [12] corresponds to very strongly damped fluctuations (cf. Fig. 1) and therefore even small variations in the slope of the distribution function results in dramatic changes to the scattering cross-section, as it is illustrated in Fig. 3. We have plotted in Fig. 3 part of the dynamical form factor (10) corresponding to the Langmuir wave resonance using modified distribution functions (18) with different exponents. Dramatic changes in the profile of $S(\mathbf{k}, \omega)$ with moderate variations of $\mu$ are not observed in the experimental results. As the TS experiments by Glenzer et al. [10] are the first accurate measurements in plasmas which should display features revealing non-Maxwellian distribution functions, their apparent absence must prompt more studies on the limits of applicability of the solutions (18) and (19).

\section{CONCLUSIONS}

We have reported on the theories relevant to the TS experiments in highly ionized laser produced plasmas which have been applied in the interpretation of experimental measurements [12]. The full account of these studies, including results of experiments and detailed comparisons with Lasnex simulations will be published elsewhere [12].

The potential of the TS can be fully explored only by involving the relevant theoretical results for the scattering cross section and dynamical form factor. We have derived and applied to the experimental conditions a Thomson scattering cross section (8) in inhomogeneous plasmas. Using local Maxwellian distribution functions we have found values of important plasma parameters by accurately matching spectra of Thomson scattered radiation, Fig. 2. A simultaneous analysis of the scattered light spectra in the frequency range corresponding to ion acoustic and Langmuir fluctuations in a hot and dense plasma 
has demonstrated means of accurate measurements of the ionization level $Z$. This could established TS as an important diagnostic in opacity studies of dense plasmas.

Finally we have compared theoretical predictions for the TS cross section for electrons described by the non-Maxwellian distribution functions with experimental results. SuperGaussian distributions are expected in plasmas with strong IB heating where $Z v_{0}^{2} / v_{e}^{2}>$ 1. Changes in the electron distribution function involve flattening of $f^{e}$ at small particle velocities and reduction of the number of fast electrons. Our comparisons show convincingly no changes in the tail of the distribution function over wide range of flow velocities, densities and electron temperature accessible in the experiment. Further studies are necessary in order to improve theoretical basis for non-Maxwellian distributions. It has been shown, for example, by theory and Fokker-Planck simulations [17] that localised heating of the plasma due to IB absorption leads to distribution functions which are linearly unstable to the return current instability. Signatures of this instability has been also observed in the experiment [12].

\section{ACKNOWLEDGMENTS}

This work was partially supported under the auspices of the U.S. Department of Energy by the Lawrence Livermore National Laboratory under contract No. W-7405-ENG-48. Part of this support was provided through the LLNL-LDRD program under the Institute for Laser Science and Applications. 


\section{REFERENCES}

* On leave from the Theoretical Physics Institute, Department of Physics, University of Alberta, Edmonton T6G 2J1, Alberta, Canada.

[1] D. D. Clayton, Principles of Stellar Evolution and Nucleosynthesis, (The University of Chicago Press, Chicago, 1983).

[2] V. N. Tsytovich, R. Bingham, U. de Angelis, and A. Forlani, J. Quant. Spectrosc. Radiat. Transfer, 55, 787 (1996); ibid, 58, 127 (1997).

[3] J. E. Bernard, H. A. Baldis, D. M. Villeneuve, and K. G. Estabrook, Phys. Fluids, 30, 3616 (1987).

[4] M. D. Tracy, J. S. DeGroot, K. G. Estabrook, and S. M. Cameron, Phys. Fluids B 4, 1576 (1992).

[5] B. LaFontaine, H. A. Baldis, D. M. Villeneuve, J. Dunn, G. D. Enright, J. C. Kieffer, H. Pepin, M. D. Rosen, D. L. Mathews, and S. Maxon, Phys. Plasmas 1, 2329 (1994).

[6] S. H. Glenzer, C. A. Back, L. J. Suter, M. A. Blain, O. L. Landen, J. D. Lindl, B. J. MacGowan, G. F. Stone, R. E. Turner and B. H. Wilde, Phys. Rev. Lett. 79, 1277 (1997).

[7] R. Teyssier, D. Ryutov, and B. Remington, ApJS, this volume (1998).

[8] S. Ichimaru, Statistical Plasma Physics, vol. I, (Addison-Wesley, Redwood City, 1992).

[9] J. D. Jackson, Classical Electrodynamics, (Wiley, New York, 1975).

[10] S. H. Glenzer, C. A. Back, K. G. Estabrook, and B. J. MacGowan, Rev. Sci. Instrum. 68, 668 (1997).

[11] D. E. Evans and J. Katzenstein, Rep. Prog. Phys. 32, 207 (1969).

[12] S. H. Glenzer, et al., in preparation (1998). 
[13] J. F. Myatt, W. Rozmus, V. Yu. Bychenkov and V. T. Tikhonchuk, Phys. Rev. E 57, 3383 (1998).

[14] A. B. Langdon, Phys. Rev. Lett. 44, 575 (1980).

[15] J-P. Matte, M. Lamoureux, C. Möller, R. Y. Yin, J. Delletrez, J. Virmont, and T. W. Johnston, Plasma Phys. Controlled Fusion, 30, 1665 (1988).

[16] J. Zheng, C. X. Yu, and Z. J. Zheng, Phys. Plasmas 4, 2736 (1997).

[17] V. T. Tikhonchuk, W. Rozmus, V. Yu. Bychenkov, C. E. Capjack and E. Epperlein, Phys. Plasmas 2, 4169 (1995). 


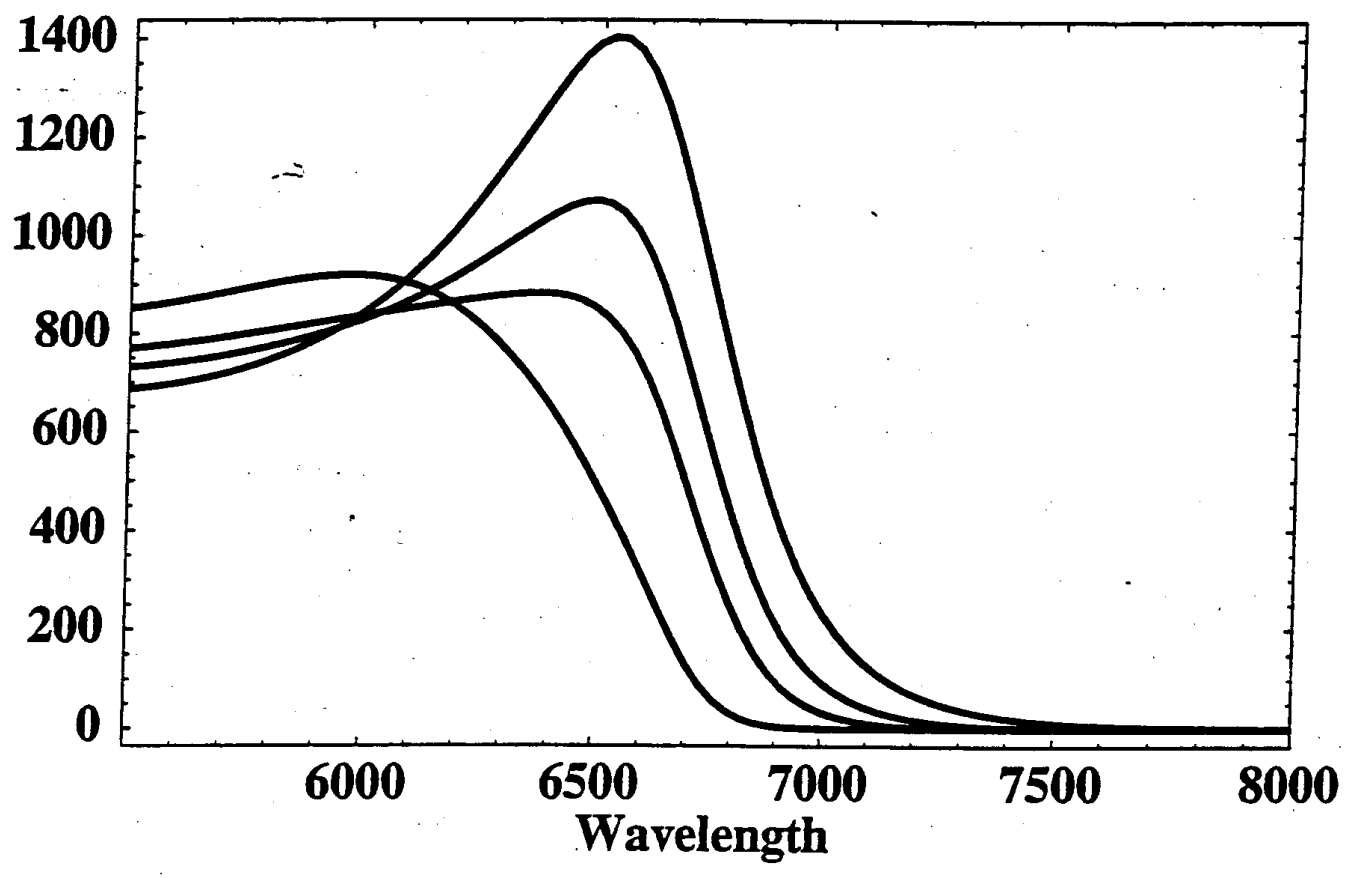




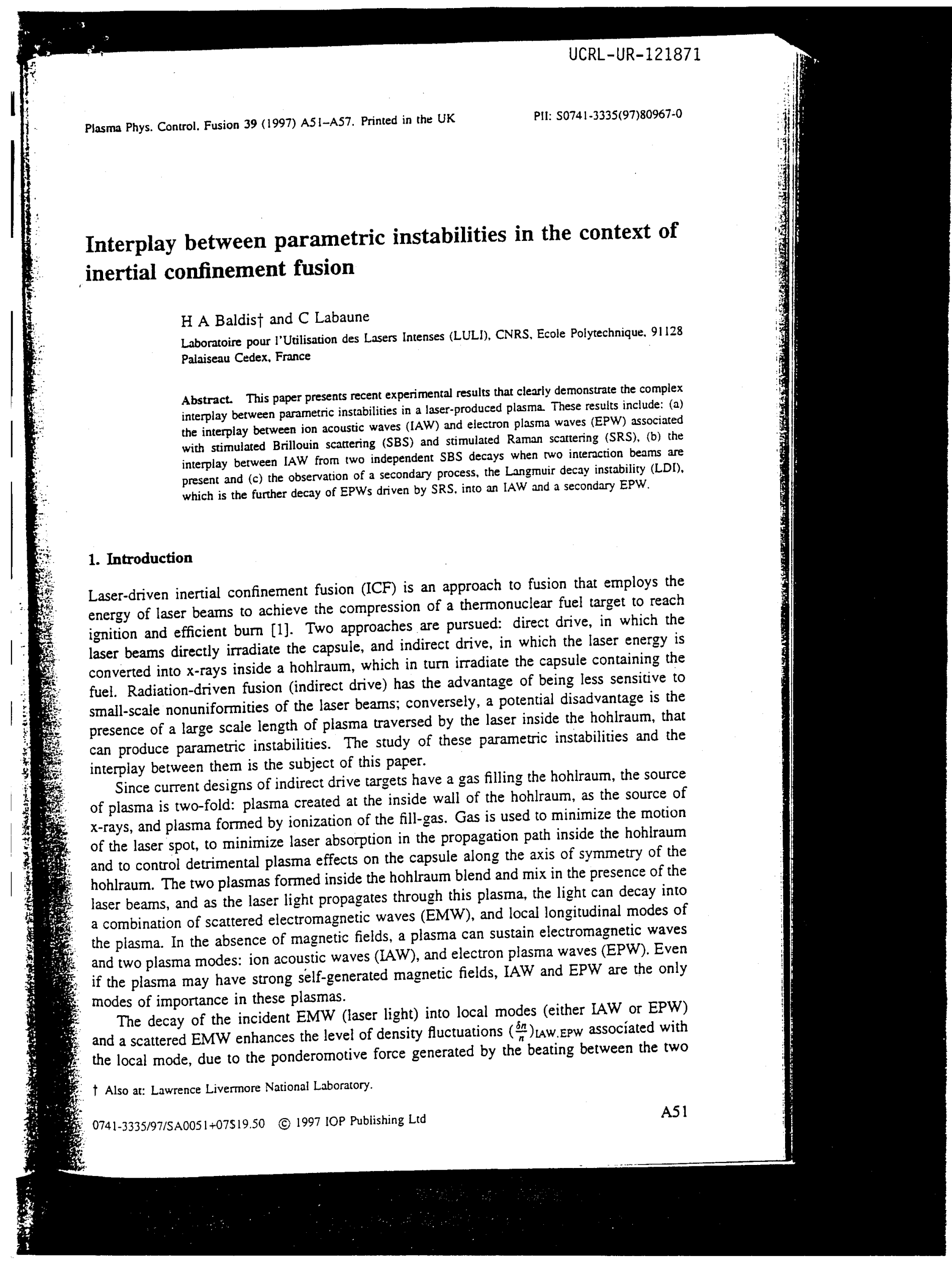


EMWs [2]. This increase of $\left(\frac{\delta n}{n}\right)_{\text {IAW.EPW further enhances the decay, thus generating a }}$ feed-back loop that yields to unstable growth, and thus to the creation of an instability. If the waves involved in the decay satisfy conservation of energy, $\omega_{0}=\omega_{1}+\omega_{2}$, and conservation of momentum, $k_{0}=k_{1}+k_{2}$ (where $l$ is the scattered EMW, 2 is either an IAW or an EPW, and $\omega$ and $k$ are the respective frequency and wavevector for each wave) the decay grows exponentially. This exponential growth is certainly a potential problem in ICF: the scattered EMW can leave the plasma, thus losing a large fraction of the laser energy, as well as producing a redistribution of the laser energy inside the hohlraum, affecting the requirements for symmetry of implosion. The instabilities of interest are stimulated Brillouin scattering (SBS), and stimulated Raman scattering (SRS) $[2,3]$. Stimulated Brillouin scattering consists of the decay of the incident EM wave $\left(\omega_{0}, k_{0}\right)$ into a scattered EMW ( $\left.\omega_{\mathrm{SBS}}, k_{\mathrm{SBS}}\right)$ and an IAW ( $\left.\omega_{\mathrm{IAW}}, k_{\mathrm{IAW}}\right)$, and stimulated Raman scattering consists of the decay of the incident EMW $\left(\omega_{0}, k_{0}\right)$ into a scattered EMW ( $\left.\omega_{S R S}, k_{\mathrm{SRS}}\right)$ and an EPW ( $\left.\omega_{\mathrm{EPW}}, k_{\mathrm{EPW}}\right)$.

These instabilities have been studied extensively in the past, by characterizing primarily the EMW scattered from the plasma [3], and in the context of ICF, the effort has been primarily on the evaluation of the reflectivity; in other words, the energy loss. Since hohlraums for the national ignition facility (NIF) are much larger than the present ScaleI Nova targets [1], an estimate of the future role of parametric instabilities requires an understanding of the behaviour of the instabilities in present targets, in order to extrapolate with confidence to larger plasmas.

A vital aspect of the evolution of instabilities is their growth in the presence of waves (either IAW or EPW) associated with another instability and, to a lesser level, in the presence of waves associated with secondary decays such as the parametric decay of the EPW from SRS. It has become clear in recent years that the interaction between different plasma waves yields an interplay between the parametric instabilities that obscures the characterization and understanding of the parametric process itseif. Waves driven by different parametric instabilities can interact among themselves, modifying in turn their behaviour, as was clearly demonstrated in early experiments at NRC in Ottawa [4,5]. A further complication arises when considering the overlap of multiple laser beams; not only is there the interplay between instabilities driven by one beam, but also the interplay between instabilities driven by different beams [6].

We present in this paper recent experimental results, obtained at the Laboratoire pour l'Utilisation des Lasers Intenses (LULI), that clearly demonstrate the complexity of the interplay between instabilities. We will discuss in the following three particular effects: (a) the interplay between IAW and EPW associated with SBS and SRS, (b) the interplay between IAW from two independent SBS decays when two interaction beams are present and (c) the observation of a secondary process, the Langmuir decay instability (LDI), which is the further decay of EPWs, driven by SRS, into an IAW and a secondary EPW.

\section{Experimental arrangement}

The results presented here were obtained using collective Thomson scattering as the key diagnostic $[7,8]$. The laser beam configuration and plasma formation was as follows. The 600 ps full-width half maximum (FWHM) Gaussian beams were all in the same plane and arrived at the target at different times: two $0.53 \mu \mathrm{m}$ plasma producing beams at $t=0$, a $0.53 \mu \mathrm{m}$ plasma heating beam at $t=1.2 \mathrm{~ns}$, the $0.35 \mu \mathrm{m}$ Thomson scattering probe beam at $t=1.48 \mathrm{~ns}$, and the $1.05 \mu \mathrm{m}$ interaction beam at $t=1.72 \mathrm{~ns}$. Two interaction beams have been used individually or together for different parts of the experiment. The 
geometry of the experiment is shown in figure 1. Random phase plates (RPP) [9] were used on the beams producing and heating the plasma to obtain plasmas that were as uniform and reproducible as possible. The interaction beam was focused with an $f / 6$ lens through an RPP of $2 \mathrm{~mm}$ square elements. The resulting focal spot diameter was $320 \mu \mathrm{m}$ FWHM with a focal depth of $1.5 \mathrm{~mm}$. The average intensity in the focal spot was $10^{14} \mathrm{~W} \mathrm{~cm} \mathrm{~cm}^{-2}$. The targets were $450 \mu \mathrm{m}$ diameter, $1.5 \mu \mathrm{m}$ thick, $(\mathrm{CH})_{n}$ disks. The electron density at the peak of the plasma profile evolved from $0.25 n_{c}$ to $0.08 n_{c}$, from the beginning to the end of the interaction beam (where $n_{\mathrm{c}}=1.1 \times 10^{21} \mathrm{~cm}^{-3}$ is the critical electron density for $\lambda_{0}=1.05 \mu \mathrm{m}$ light). The density profile had a quasi-inverse-parabolic form along the interaction axis, with an FWHM of $1 \mathrm{~mm}$. The electron temperature $\left(T_{e}\right)$ of the plasma, obtained by Thomson scattering in the absence of the interaction beam, was between 0.4 and $0.5 \mathrm{keV}$.

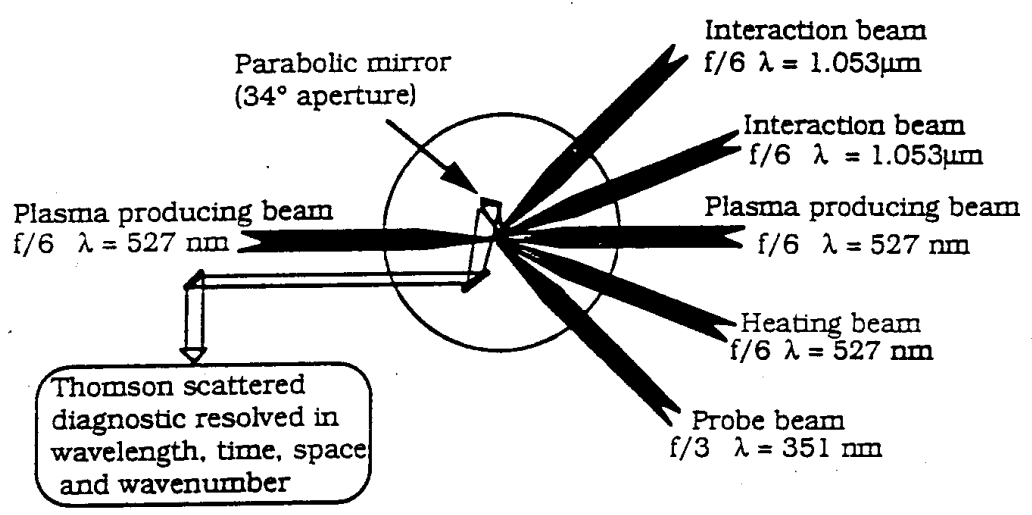

Figure 1. Experimental arrangement for the experiment at LUL1. All laser beams and Thomson scartering collecting optics are in the same plane.

The geometry for Thomson scattering permitted us to collect scattered light corresponding to IAW and EPW associated with SBS and SRS over a large range of scattering angles, thus permitting the observation of waves associated with backscattering as well as sidescattering instabilities. The Thomson scattering probe was focused with a combination of lens and an RPP with elongated elements, to form a focal region $100 \mu \mathrm{m}$ by $1 \mathrm{~mm}$ along the axis of the interaction beam, thus allowing us to image the location of the waves over the whole interaction length. Thomson-scattered light was collected with a parabolic mirror and imaged onto different diagnostics, using masks to select the waves of interest at different stages during the experiment.

\section{Experimental results on interplay}

\subsection{Interplay between instabilities: competition SBS-SRS}

The first result is the clear evidence of the mutual exclusion between IAW and EPW in time. Although these waves were observed to occur around the same region of plasma, they were never present at the same time. Figure 2 shows the temporal evolution of IAWs and EPWs. The scattered signals are related to the density fluctuations $\left(\frac{\delta n}{n}\right)_{\text {IAW.EPW }}$ associated with the IAWs and EPWs corresponding to backscattered SBS and SRS, respectively. If the scattering 
takes place from coherent plasma waves, the scattered power levels are proportional to the

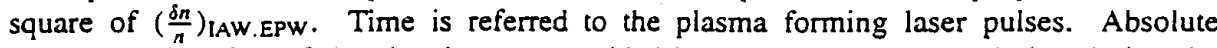
timing and location of the signals were provided by the early plasma emission during the preforming pulses. The time-resolved signals in figure 2 are from waves located $300 \mu \mathrm{m}$ from the centre of the plasma in the direction of the interaction laser beam. The location of these waves is characteristic of the plasma and laser parameters, and is discussed in [10]. The interaction region is smaller than the scale length of the plasma and is not limited by the length of the line focus of the Thomson scattering probe.

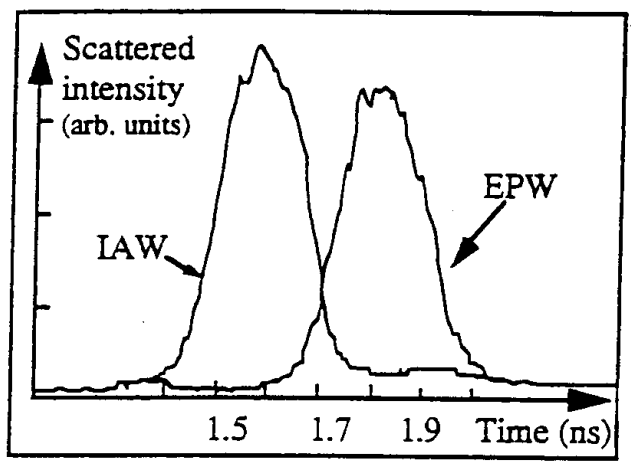

Figure 2. Temporal evolution of Thomson-scattered light from IAWs and EPWs, associated with backscattered SBS and SRS. respectively, from the same region of plasma, and recorded on the same streak camern. The Thomson-scamered intensity is proportional to the square of the levels of the density fluctuations associated with the plasma waves.

The onset of the IAWs takes place at the very beginning of the interaction pulse; the emission lasts around 300 ps and is maximum $\sim 100$ ps before the peak of the interaction pulse. Simultaneously with the disappearance of the IAWs, one observes a rapid growth in the level of fluctuations associated with EPWs. The EPWs are observed only after the disappearance of the IAWs, the duration of the EPWs' signal is around 300 ps. Further to the temporal interplay between IAWs and EPWs, we observe that, within $100 \mu \mathrm{m}$ (which represents shot to shot fluctuations), the EPWS are located in the same region of plasma as the IAWs just disappeared from. This interplay between the two instabilities was observed in all laser shots, and under various plasma conditions.

\subsection{Multiple beam effects: resonance and off-resonance IAW behaviour}

A different aspect of the experiment was the study of the IAW when more than one interaction beam was present [6]. For this study, a second interaction laser beam was used at a lower intensity than the primary interaction beam, to act as a seed of IAW for the primary interaction beam. This had the effect of modifying the growth of SBS, which then starts from a well defined level of IAW, rather than from thermal fluctuations. The angle between the two beams was $22.5^{\circ}$. Ion acoustic waves travelling along different directions were recorded with temporal and spatial resolution. The direction of observation was defined by masks on the Thomson scattering optics, limiting the collection angle to $5^{\circ}$, and in steps of $5^{\circ}$. An interesting aspect of this configuration is that of all possible IAWs driven by the seed beam, only side scattering SBS with IAW travelling along the direction bisecting the angle between the two beams will have matching wavenumber to a similar 
decay pumped by the main interaction beam. Along any other direction, there is a mismatch between the IAW wavenumbers driven by each of the two beams.

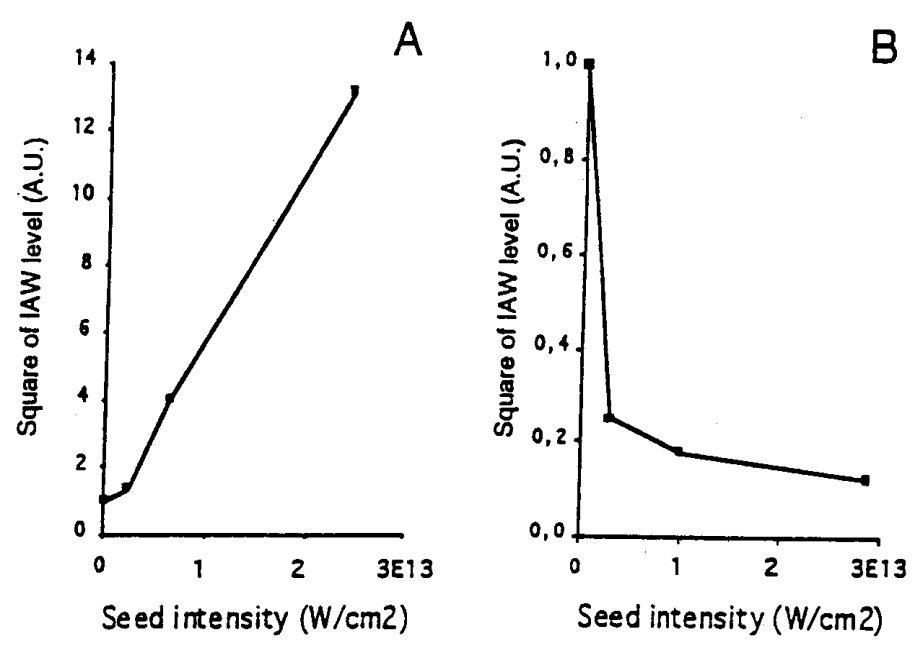

Figure 3. Levels of intensities of Thomson-scattered light from ion acoustic waves as a function of seed-beam intensity, integrated in time and space. (A) lon acoustic waves travelling along the bisector to the directions of the pump and seed beams (resonance). (B) Ion acoustic waves along a direction off-resonance.

Along the direction of matching wavenumbers a strong enhancement in the level of LAWs was observed. The total signals, integrated in time and space, are plotted in figure 3(A) as a function of the seed-beam intensity, showing enhancement of LAW even for a low-intensity seed beam. An unexpected result was obtained when probing along off-resonance directions. Even for small levels of seed beam $\left(I_{\text {seed }}=10^{13} \mathrm{~W} \mathrm{~cm}^{-2}\right.$ ), a strong reduction was observed in the level of IAW. A further reduction was obtained as the intensity of the seed beam was increased, with a total reduction of a factor of eight at $I_{\text {seed }}=3 \times 10^{13} \mathrm{~W} \mathrm{~cm}^{-2}$. Figure $3(\mathrm{~B})$ shows the intensities of scattered light from LAW for this off-resonance direction, integrated in time and space as a function of seed beam intensity, showing the drastic effect on the IAW. A ratio of $I_{\text {seed }} / I_{\text {pump }}=3 \%$ was sufficient to reduce the total observed level of scattered light from IAW by a factor of five. The reduction in the level of scattered light from IAW was observed on either side of the direction of resonance. At $5^{\circ}$ away from resonance, the mismatch in wavevector between the seed and the pump beams is sufficient to change a strong enhancement into a strong reduction in the level of $\mathrm{LAW}$. For a low seed beam intensity $\left(I_{\text {seed }} / I_{\text {pump }}=3 \%\right.$ ), the amount of reduction off-resonance is a much larger effect than the enhancement at resonance. An explanation for the reduction of IAW is the presence of IAWs driven by the seed beam, either in backscatter or sidescatter, which are seen by the pump beam as long wavelength ion waves [6]. Modification to the level of scattered SBS light is discussed by Labaune et al [11].

\subsection{Secondary decay processes: Langmuir decay instability}

The study of the interplay between instabilities is further complicated by secondary decay processes. This can be the decay of the EMW associated with either SBS or SRS, or the 
decay of the IAW or EPW into lower modes, yielding in some cases to cascading effects. Of current interest is the Langmuir decay instability (LDI), where an EPW (Langmuir wave) decays into a secondary EPW and an IAW $[12,13]$. If SRS generates large levels of EPW, this can become a source to drive LDI. Since one of the daughter waves of the decay is an LAW, processes that can modify the levels of LAW can in turn modify the LDI decay, which in turn can have an effect on the initial SRS decay. A consequence of this interplay is the potential for providing a saturation mechanism for SRS [14]. Recent simulations have indicated this possibility [15-17].

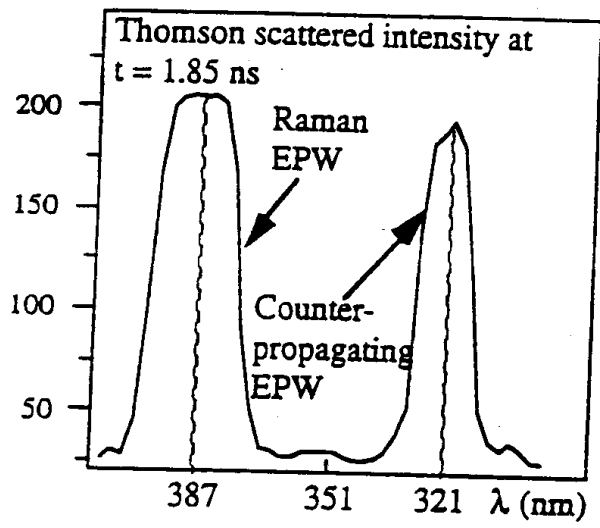

Figure 4. Thomson-scartering spectra showing two sets of electron plasma waves travelling in opposite directions. Different attenuarions have been used for each wave, resulting in a relative amplitude $\delta n / n$ (down) $/ \delta n / n$ (up) of approximately 20 to 40 .

Direct evidence for the presence of $L D I$ has been obtained under experimental conditions as described in the previous sections. Using space- and time-resolved Thomson scattering, two sets of EPW were observed, one travelling parallel to the incident laser puise, and the second travelling in the opposite direction. Figure 4 shows Thomson scattering spectra at time $t=1.85 \mathrm{~ns}$. The signals correspond to Thomson scattered light from EPW having the same frequency, but opposite wavevector. The observed spectra of these two sets of EPW are symmetric with respect to the probe frequency, and are in perfect synchronization. Each wave is recorded with different attenuation, resulting in a relative amplitude $\delta n / n$ (down) $/ \delta n / n$ (up) of approximately 40 . Both waves are present at the same location in the plasma.

The observed up and down EPWs can only be explained by LDI. Reflection of the EPWs at their critical density is ruled out because of the strong damping affecting the EPW. Coupling to IAW driven by SBS is also ruled out because of the time difference between SBS and SRS, as demonstrated in section 3.1.

\section{Conclusions}

We have presented clear evidence of three different aspects of the interaction between waves associated with different parametric instabilities. These results show the complexity of the interaction process, and the need to study these instabilities together. Results show the strong effect of IAWs, not only on SRS but on SBS as well. The interplay between SBS and SRS 
confirms early results [4] of this phenomena; the reversed temporal behaviour between SBS and SRS in the early experiment is due to the different plasma and laser conditions. The coupling between IAWs driven by SBS from two different interaction beams is a new result that shows, for the first time, the reduction of SBS due to IAW from a different SBS decay. This indicates the complexity in dealing with experiments where multiple beams overlap. Finally, the observation of counter-propagating EPW from SRS clearly demonstrates the existence of LDI, a mechanism that introduces a further link between EPWs and LAWs.

\section{Acknowledgments}

The authors would like to thank B Bauer, N Renard, E Schifano and A Michard for their participation during the experiments, and V T Tikhonchuk, W Rozmus, W L Kruer and $G$ Laval for useful discussions. This work was partially supported under the auspices of the US Department of Energy by the Lawrence Livermore National Laboratory under contract No W-7405-ENG-48.

\section{References}

[1] Lindl J 1995 Phys. Plasmas 23933

(2) Kruer W L 1988 The Physics of Laser Plasma Interactions (Redwood City: Addison-Wesley)

[3] Baldis H A. Campbell E M and Kruer W L 1991 Laser-plasma interactions Physics of Laser Plasma ed A Rubenchik and W Witkowski (Amsterdam: North-Holland)

[4] Walsh C J, Villeneuve D M and Baidis H A 1984 Phys. Rev. Lerr. 531445

[5] Villeneuve D M. Baldis H A and Bernard J 1987 Phys. Rev. Lerr. 591585

[6] Baldis H A. Labaune C, Schifano E, Renard N and Michard A 1996 Resonant seeding of stimulated Brillouin scantring by crossing laser beams 1996 Phys. Rev. Lest. 772957

[7] Labaune C, Baldis H A, Renard N, Schifano E, Baton S, Michard A. Seka W, Bahr R E. Bauer B, Baker K and Estabrook K 1995 Phys. Rev. Lett. 75248

[8] Baldis H A and Labaune C 1996 Rev. Sci. Instrum. 67451

[9] Kato Y, Mima K. Miyanaga N, Arinaga S, Kitagawa Y. Nakatsuka M and Yamanaka C 1984 Phys. Rev. Lett. 531057

[10] Tikhonchuk V T, Labaune C and Baldis H A 1996 Phys. Plasmas 33777

[11] Labaune $C$ and Baidis H A 1997 Stimulated Brillouin scattering measurements in a laser-produced plasma this conference

[12] Baker K L et al 1996 Phys. Rev. Letr. 7767

[13] 1995 LULI Rapport Annuel p 60

[14] Drake R P and Batha S H 1991 Phys. Fluids B 32936

[15] Bonnaud G, Pesme D and Pellat R 1990 Phys. Fluids B 2161

[16] Bezzerides B. DuBois D F and Rose H A 1993 Phys. Rev. Lert. 702569

[17] Kolber T, Rozmus W and Tikhonchuk V 1993 Phys. Fluids B 5138 
UCRL-UR-121872

EUROPHYSICS LETTERS

1 April 1997

Europhys. Lett., 38 (1), pp. 31-36 (1997)

\title{
Interpretation of stimulated Brillouin scattering measurements based on the use of random phase plates
}

\author{
C. Labaune ${ }^{1}$, H. A. Baldis ${ }^{1}\left({ }^{*}\right)$ and V. T. TikhonchuK ${ }^{2}$ \\ 1 Laboratoire pour l'Utilisation des Lasers Intenses, Ecole Polytechnique \\ Centre National de la Recherche Scientifique, 91128 Palaiseau cedex, France \\ ${ }^{2}$ P. N. Lebedev Physics Institute, Russian Academy of Science, Moscow 117924, Russia
}

(received 9 September 1996; accepted in final form 25 February 1997)

PACS. 52.35Fp - Electrostatic waves and oscillations (e.g., ion-acoustic waves).

PACS. 52.35Mw- Nonlinear waves and nonlinear wave propagation (including parametric effects, mode coupling, ponderomotive effects, etc.).

PACS. 52.40Nk - Laser-plasma interactions (e.g., anomalous absorption, backscattering, magnetic field generation, fast particle generation).

\begin{abstract}
Experimental reflectivities due to stimulated Brillouin scattering (SBS) and ion acoustic fluctuations have been successfully modelled, over four orders of magnitude of SBS reflectivity, using a newly developed theory of parametric instabilities in randomized laser beams. The observed temporal evolution of SBS has been interpreted using the hydrodynamic evolution of the plasma parameters. Location of SBS in the front part of the plasma is due partly to pump depletion in the SBS-active speckles, and partly to plasma conditions.
\end{abstract}

In the last two decades, stimulated Brillouin scattering (SBS) has received considerable experimental and theoretical attention [1], [2]. In this process the incident light wave decays into an ion acoustic wave (IAW) and a scattered electromagnetic wave. This instability can grow in the plasma corona which surrounds the fuel pellet in laser fusion experiments. It can reduce the coupling efficiency of laser light into the plasma and destroy the high degree of symmetry necessary for efficient compression of the capsule [3]. Extrapolation of present results to Inertial Confinement Fusion (ICF) targets requires understanding of the instability. This will come from modeling which can provide good agreement with experiments on more than one features, including SBS reflectivity and evolution of the waves involved in the coupling.

This letter presents the application of a novel technique for modeling the interaction physics, which takes into account the statistics of the distribution of laser light intensity in speckles in a plasma and employs a consistent description of SBS reflectivity from a single speckle. This model, described in more detail in ref. [4], predicts SBS reflectivities from a recent experiment, over four orders of magnitude, without any adjustments in the initial noise level of the density

( $\left.{ }^{*}\right)$ Present address: Institute for Laser Science and Applications (ILSA), Lawrence Livermore National Laboratory, POB 808, Livermore CA 94550, USA.

(C) Les Editions de Physique 
fluctuations from which SBS grows. Apart from two very recent experiments [5], [6], modeling of SBS reflectivities without any adjustment factor always failed in the past twenty years. The success of the present comparison is due to the evolution of both experimental and theoretical approaches. Ameliorations in the experimental part consist of a combination of improved use of Thomson scattering [7] to diagnose the decay waves and the use of a well-characterized laser intensity distribution in the focal volume, obtained with a random phase plate (RPP) [8]. Amelioration in the theoretical part is the application of the statistics of randomly distributed independent speckles [9] to the SBS theory in an individual speckle including effects of light diffraction and depletion, and plasma inhomogeneity [10]. Because of small size and random location of speckles, experimental observations represent averaged data, which are the sum of emissions from many independent speckles integrated over the instrumental resolution time and volume. The stimulated scattering from an individual speckle could be very strong and nonlinear effects like the pump depletion define the single speckle reflectivity. However, the number of statistically significant speckles in the interaction volume may be relatively small having a minor effect on the overall beam propagation through a plasma. Thus the conception of randomly distributed independent speckles makes a natural bridge between strong nonlinear effects observed in experiments and relatively weak overall modification of the interaction beam.

The experimental results presented in this paper have been produced by the interaction between an RPP smoothed laser beam and a well-characterized preformed plasma [11]. The intensity of the interaction beam was intentionally kept low to produce SBS in a weak-coupling regime, to be below saturation, to have a number of active speckles small enough so they can be considered as independent, with a total number of speckles large enough so the statistics can be applied. Separate shots have shown that the speckles distribution was not much modified by the presence of the plasma compared to the distribution in the vacuum. To isolate SBS from some other parametric instabilities which can grow in the corona, the electron density of the preformed plasma was chosen below quarter critical ( $n_{\mathrm{c}}$ is the critical electron density for the interaction beam) during the interaction pulse. The low laser intensity, not far above SBS threshold, should also allow to separate SBS and filamentation, as this process has a higher threshold than SBS.

The experiments have been performed using the six beams of the Laboratoire pour l'Utilisation des Lasers Intenses (LULI) laser facility. The interaction beam had a wavelength of $\lambda_{0}=1.053 \mu \mathrm{m}$, a pulse duration of $0.6 \mathrm{~ns}$ (FWHM) and was focused with an $f / 6$ lens combined with RPP. The focal spot diameter was $320 \mu \mathrm{m}$, and the maximum average intensity was $10^{14} \mathrm{~W} / \mathrm{cm}^{2}$, for an energy of $100 \mathrm{~J}$. The Rayleigh length, $L$, of the beam was $\sim 1 \mathrm{~mm}$. The mean diameter of the speckles is $a_{0} \sim 6 \mu \mathrm{m}$ and the mean length is $L_{\mathbf{R}} \sim 300 \mu \mathrm{m}$. These dimensions correspond to the decorrelation distances between different speckles. Speckles were well characterized everywhere in the plasma. The plasma was preformed by exploding a thin small disk of plastic $(\mathrm{CH})_{n}$ with two opposite laser beams with a wavelength of $526 \mathrm{~nm}$, arriving to the target $1.72 \mathrm{~ns}$ before the interaction beam. The electron density at the peak of the plasma profile decayed exponentially with the characteristic time $0.4 \mathrm{~ns}$. It evolved from $0.2 n_{\mathrm{c}}$ to $0.07 n_{\mathrm{c}}$, from the beginning ( $\left.t=1.7 \mathrm{~ns}\right)$ to the end $(t=2.3 \mathrm{~ns})$ of the interaction pulse (where $n_{c}=1.1 \times 10^{21} \mathrm{~cm}^{-3}$ is the critical electron density for $\lambda_{0}=1.05 \mu \mathrm{m}$ light). The density profile had a quasi-inverse-parabolic form along the interaction axis, with a characteristic length $L_{n}=1 \mathrm{~mm}$. The electron temperature $T_{\mathrm{e}}$ of the plasma before the interaction beam arrival, was between 0.4 and $0.5 \mathrm{keV}$, which increased during the interaction pulse to about $0.6-0.7 \mathrm{keV}$. The ion temperature $T_{\mathrm{i}}$ was approximately $250 \mathrm{eV}$. The electron densities and temperatures, obtained by Thomson scattering, agree well with $2 \mathrm{D}$ hydrodynamic simulations of the plasma evolution. These simulations provide also values of the plasma expansion velocity $u(z)$, its scale length $L_{u}$ changes from $300 \mu \mathrm{m}$ in the plasma center up to $500 \mu \mathrm{m}$ at the plasma edges. Both the 
ion acoustic and electromagnetic waves produced by the SBS decay in backscattering direction were diagnosed. The thomson scattering of a $351 \mathrm{~nm}$ probe beam was used to measure the ion fluctuation levels as a function of space and time. The backscattered light into the focusing optics of the interaction beam $(\delta \Omega=0.045 \mathrm{sr})$ was spectrally resolved as a function of time, and absolute reflectivities were measured. Detailed results are presented in ref. [12].

Experimental SBS reflectivities depended strongly on the average incident laser intensity: from $7 \times 10^{-7}$ at $4 \times 10^{12} \mathrm{~W} / \mathrm{cm}^{2}$, to $3 \times 10^{-2}$ at $8 \times 10^{13} \mathrm{~W} / \mathrm{cm}^{2}$. Laser intensities have been modified by changing the laser energies and keeping all plasma conditions the same. Another major result of this experiment is the direct observation of the location of the ion acoustic waves associated with SBS by imaging the plasma using the Thomson scattered light on a streak camera. Significant level of ion acoustic fluctuations is observed only in a small region of the density profile located in the front part of the plasma relative to the interaction beam. The measured scattered power, at maximum intensity, corresponds to an average density fluctuation level $\left(\delta n / n_{\mathrm{e}}\right)^{2}$ of $\sim 5 \times 10^{-4}$. The duration of the Thomson scattered signal has a full width at half-maximum of $\sim 300$ ps. It is shorter than the interaction pulse, with its maximum at $\sim 100$ ps before the maximum of the interaction pulse. Measurements of the temporal evolution and of the spectral characteristics of the SBS backscattered light agree with the observed behavior of the ion acoustic waves [12].

The modeling is based on the theory of stationary SBS convective amplification in an inhomogeneous plasma [13] which is applied to an individual speckle to calculate the SBS reflectivity and IAW amplitude. We assume that SBS starts from the thermal noise level of IAW fluctuations and due to the plasma flow velocity gradient each particular three wave interaction extends over a distance $L_{\mathrm{a}}$ which is short compared to the Rayleigh length $L_{\mathrm{R}}$ of the speckle. Then we solved the equation for the laser power in a speckle accounting for the losses due to the scattered light generation and found the transmitted power, $p_{\mathrm{t}}$ as a function of incident power $p_{\mathrm{i}}$. The SBS reflectivity from a speckle was defined as the lost pump power, $R=\left(p_{\mathrm{i}}-p_{\mathrm{t}}\right) / p_{\mathrm{i}}$, it was found as a function of $p_{\mathrm{i}}$ and then averaged over the distribution of speckles using the statistical description of ref. [9], yielding $\langle R\rangle$, the average reflectivity from a distance of one Rayleigh length.

The total reflectivity is calculated by integration of $\langle R\rangle$ along the laser ray trajectories taking into account the profiles of plasma density, temperature, -velocity, and laser intensity, provided by $2 \mathrm{D}$ hydrodynamical simulations. The equation for the average intensity of the laser beam is .

$$
\frac{\mathrm{d}\langle I\rangle}{\mathrm{d} z}=-\frac{2 z}{z^{2}+L^{2}}\langle I\rangle-\kappa_{\mathrm{iB}}\langle I\rangle-\frac{\langle I\rangle}{L_{\mathrm{R}}}\langle R\rangle,
$$

where $\kappa_{\mathrm{iB}}$ is the inverse bremsstrahlung absorption coefficient. The first term in the right-hand side accounts for the divergence of the full laser beam, with the two other terms describing losses due to absorption and scattering, respectively. Defining the beam power $\langle P\rangle=\pi \rho^{2}\langle I\rangle$, where $\rho(z)$ is the beam radius given by geometrical optics, we solve eq. (1) for the given density profile, assuming that the input power $\left\langle P_{\mathrm{i}}\right\rangle$ at $z=-L_{n}$ is known, and we calculate the scattered power, which is defined as $\left\langle P_{\mathrm{s}}\right\rangle=\int_{-L_{n}}^{L_{n}} \frac{\mathrm{d} z}{L_{\mathrm{R}}} \pi \rho^{2}\langle R\rangle\langle I\rangle$.

TABLE I. - Measured and calculated total SBS reflectivities for various incident laser intensities.

\begin{tabular}{lllll}
\hline Average laser intensity (W/cm & \\
Total measured SBS reflectivity & $8 \times 10^{12}$ & $2 \times 10^{13}$ & $4 \times 10^{13}$ & $8 \times 10^{13}$ \\
Total calculated reflectivity & $10^{-6}$ & $10^{-5}$ & {$[2-4] \times 10^{-4}$} & {$[1-3] \times 10^{-2}$} \\
& $8 \times 10^{-7}$ & $2 \times 10^{-6}$ & $3 \times 10^{-4}$ & $1.3 \times 10^{-2}$ \\
\hline
\end{tabular}




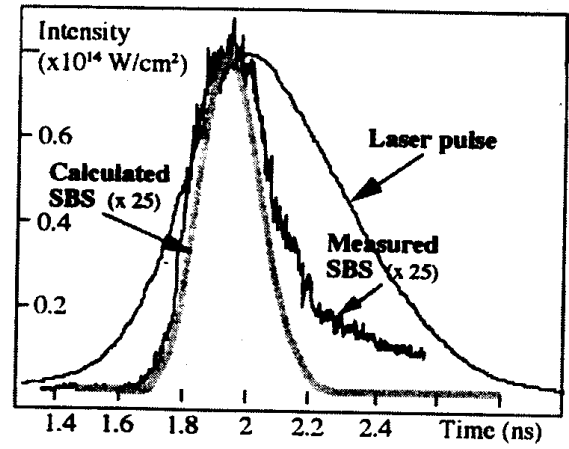

Fig. 1. - Calculated and measured temporal dependence of the SBS intensity, relative to the incident laser pulse. The incident laser intensity is $0.8 \times 10^{14} \mathrm{~W} / \mathrm{cm}^{2}$.

Figure 1 shows the comparison between calculated and measured SBS intensities as a function of time. Both the experiment and the calculation show that the maximum of SBS emission occurs $\sim 100 \mathrm{ps}$ in advance of the laser peak because of plasma density decay, with a duration of approximately half of the incident pulse duration. This comparison is within an error bar of 50 ps. The SBS pulse shape and calculated reflectivity of $2 \%$ agree well with the measurements. Good agreement has been found also for the threshold of SBS and for the SBS reflectivities obtained for various laser intensities between $8 \times 10^{12}$ and $10^{14} \mathrm{~W} / \mathrm{cm}^{2}$, as shown in table I.

Figure 2 shows the experimental (left) and calculated (right) spatial distributions of the square of the ion acoustic amplitude $\left(\delta n / n_{\mathrm{e}}\right)^{2}$ along the axis of the interaction beam as a function of time. The spatial profile of the average ion acoustic wave amplitude $\frac{\delta n}{n_{\mathrm{e}}}(z)$ is calculated from the average reflectivity coefficient: $\left(\frac{\delta n}{n_{\mathrm{e}}}\right)^{2}=\frac{\omega_{\mathrm{s}}}{8 \pi \gamma_{\mathrm{s}}} \frac{\lambda_{0}}{L_{\mathrm{R}}}\langle R\rangle \frac{\langle I\rangle}{c n_{\mathrm{e}} T_{\mathrm{e}}}$, where $\gamma_{\mathrm{s}}$ and $\omega_{\mathrm{s}}$ are the ion acoustic damping rate and frequency. The calculated and measured levels of density fluctuations agree within a factor of two. The shift of the location of the peak of the ion acoustic waves is due to the combination of the inverse bremsstrahlung absorption of averaged laser

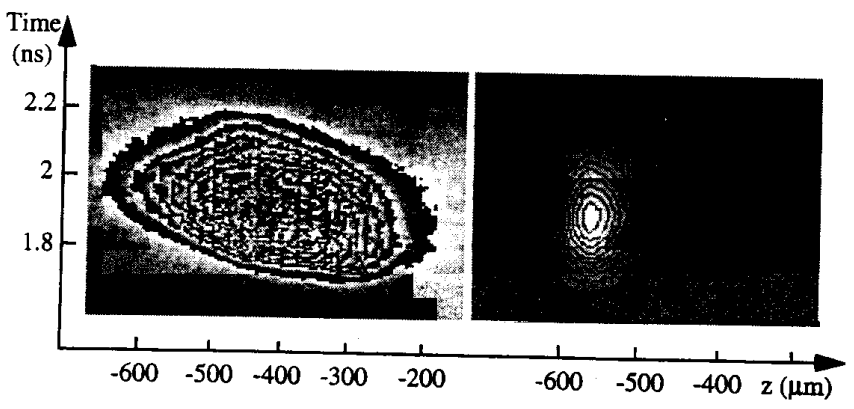

Fig. 2. - Experimental (left) and theoretical (right) distributions of the square of the density fluctuations associated with the ion acoustic waves as a function of position along the axis of the interaction beam and as a function of time. The interaction beam is coming from the left; $z=0$ corresponds to the initial target position. The average laser intensity is $0.8 \times 10^{14} \mathrm{~W} / \mathrm{cm}^{2}$. 
beam, decrease of electron temperature and change of the flow velocity gradient at a distance about $400 \mu \mathrm{m}$ from the plasma center, and pump depletion in speckles due to SBS. Note that, major contribution to the SBS reflectivitylar experiment was relatively small, $\langle G\rangle \leq 3$, the above average, corresponding to occurs inside the speckle, reducing the pain of about 11 . In such conditions pump depletion shifting the location of maximum IAW pump power along it. This reduction has the effect of shift of $0.3 L_{\mathrm{R}} \sim 100 \mu \mathrm{m}$ is achieved for SBS plasma profiles may improve the quantitativ speckle gain $G_{0} \sim 12$. Detailed description of the acoustic waves.

The direct observation of the growth of the IAWs in the front part of the de speculations made in many other experiments This paper provides a physical interiments [14] that have shown blue-shifted backward SBS. plasma based on hydrodynamical plasmation of the location of SBS in the front part of the speckles. We cannot rule out plasma profiles and on pump depletion in the SBS active lead to an increased SBS gain in nonuniform expansion of multispecies plasmas can also incorporated in the statistical SBS the front part of the plasma [15]. This effect can be also of the SBS emission from laser hot spots powever, our analysis demonstrates that assumption low laser intensities. We would like to stress out that the usual approach based on the calculation of the SBS
reflectivity from the average SBS rwo-three orders of magnitude lower convective gain, $\langle G\rangle \leq 3$, provides a reflectivity of about observed shift of the ion waves location as measured. Furthermore, it will never predict the effect will be negligible. This model is quite this shift is partly due to pump depletion and this experiments with low intensities with quite general and should be able to explain many other

We thank B. BaUer, A. MICHARD, N. RENARD, and E. SCHIFANo for their participation during the experiment, and K. ESTABROOK, D. PESME and P. MOUNAIX for useful discussions. This work was partly supported by the Russian Foundation for Basic Research (grant No. Energy by the Part of this work was performed under the auspices of the US Department of Energy by the Lawrence Livermore National Laboratory under Contract No. W-7405-Eng-48.

\section{REFERENCES}

[1] Kruer W. L., The Physics of Laser Plasma Interactions (Redwood City, California, Addison-
Wesley Publishing Company) 1988 .

[2] Baldis H. A, Campbell E. M. and Kruer W. L., Physics of Laser Plasmas (North-Holland,
Amsterdam) 1991, pp. 361-434.

[3] Lindl J., Phys. Plasmas, 2 (1995) 3933.

[4] Tikhonchuk V. T., Labaune C., Baldis H. A., Phys. Plasmas, 3 (1996) 3777

[5] Young P. E., Phys. Rev. Lett., 73 (1994) 1939.

[6] Drake R. P., Watt R. G. and Estabrook K., Phys. Rev. Lett., 77 (1996) 79.

[7] Baldis H. A., Villeneuve D. M. and Walsh C. J., Can. J. Phys., 64 (1986) 961. manaka C., Phys. Rev. Lett., 53 (1984) 1057.

[9] Rose H. A. and DuBors D. F Phys. 1057.

Phys. Rev. Lett., 72 (1994) 2883; Rys. Fluids B, 5 (1993) 590; Rose H. A. and DuBors D. F.,

[10] Tikhonchuk V. T., Pesme

hot spots in an inhomogeneous plasma, to be published in Phys. Plasmos reflectivity from laser 
[11] Seka W., Craxton R. S., Labaune C., Baldis H. A., Renard N., Schifano E. and Michard A., Bull. Am. Phys. Soc., 40 (1995) 11, 1777.

[12] Labaune C., Baldis H. A., Schifano E., Bauer B., Michard A., Renard N., Seka W., Moody J. D. and Estabrook K. G., Phys. Rev. Lett., 76 (1996) 3727.

[13] Gorbunov L. M. and Polyanichev A. N., Sov. Phys. JeTP, 47 (1978) 290; Ramani A. and MAX C. E., Phys. Fluids, 26 (1983) 1079.

[14] Mostovych A. N. et al., Phys. Rev. Lett., 59 (1987) 1193; TANAKa K. et al., Phys. Fluids, 27 (1984) 2960; Phillion D. et al., Phys. Rev. Lett., 39 (1977) 1529; Rosen M. D. et al., Phys. Fluids, 22 (1979) 2020; Young P. E. et al., Phys. Fluids B, 3 (1991) 1245; Chirokikн A. V. et al., Bull. Am. Phys., 38 (1993) 1934.

[15] Young P. E., Foord M. E., Maximov A. V. and Rozmus W., Phys. Rev. Lett., 77 (1996) 1278. 


\title{
Localization of Stimulated Brillouin Scattering in Random Phase Plate Speckles
}

\author{
H. A. Baldis, ${ }^{2}$ C. Labaune, ${ }^{1}$ J. D. Moody,${ }^{3}$ T. Jalinaud,,${ }^{1} *$ and V. T. Tikhonchuk ${ }^{4, \dagger}$ \\ ${ }^{\prime}$ Laboratoire pour l'Utilisation des Lasers Intenses, Ecole Polytechnique, Centre National de la Recherche Scientifique, \\ 91128 Palaiseau cedex, France \\ ${ }^{2}$ Institute for Laser Science and Applications (ILSA), Lawrence Livermore National Laboratory, \\ P.O. Box 808, Livermore, California 94550 \\ ${ }^{3}$ Lawrence Livermore National Laboratory, P.O. Box 808, Livermore, California 94550 \\ ${ }^{4}$ Centre de Physique Théorique, Ecole Polytechnique, Centre National de la Recherche Scientifique, \\ 91128 Palaiseau cedex, France \\ (Received 22 September 1997)
}

\begin{abstract}
This paper presents the first experimental evidence of the transverse localization of stimulated Brillouin scattering (SBS) emission to the laser beam axis, demonstrating that only a few small regions of plasma contribute to the emission. As a consequence, SBS reflectivity of these regions is much higher than the average SBS reflectivity, by a factor of $\sim 50-100$. These observations are consistent with the recent concept of SBS growing mainly in "active" speckles, having an intensity higher than the average laser intensity. [S0031-9007(98)05456-8]
\end{abstract}

PACS numbers: 52.35.Nx, 52.35.Fp, 52.40.Nk, 52.50.Jm

Stimulated Brillouin scattering (SBS) is a parametric instability which can occur during the propagation of an intense laser beam through a plasma in the presence of ion density fluctuations $[1,2]$. This instability is of crucial importance in inertial confinement fusion (ICF) because it can induce significant losses of the incident laser energy and spoil the illumination symmetry required to reach good implosion efficiency of the pellet. Stimulated Brillouin scattering is the resonant decay of the incident electromagnetic wave (EMW) into a scattered EMW and an ion acoustic wave (IAW). Characterization of the total, or macroscopic, SBS reflectivity is important to ICF, but in spite of the efforts during the last twenty years, it has been very difficult to match measured and calculated SBS reflectivities averaged over space and time. More detailed characterization of local SBS reflectivity is extremely important if one is to extrapolate with confidence the role of scattering instabilities to the large plasmas encountered in the national ignition facility targets [3], and to develop methods for controlling them.

We present in this paper the first experimental evidence of the localization of SBS emission, in a plane perpendicular to the laser beam axis, demonstrating that only a few small interaction regions contribute to the total SBS light. SBS emission from these small spots has been evaluated leading to local reflectivities much higher than the average SBS reflectivity, by a factor of $\sim 100$. These observations are consistent with the recent concept of convective amplification of SBS in randomly distributed speckles [4] as produced by a random phase plate (RPP) [5]. This new approach is important as it can explain the moderate observed average SBS reflectivities because of the limited number of SBS-active speckles, and pump depletion within these speckles. Application of the statistical theory has been successful in explaining a number of different physical features of SBS in a recent experiment [6]. The obser- vation of localized regions of SBS emission presented in this paper demonstrates the importance of the microscopic description of SBS.

The use of a RPP on the beam which pumps the SBS instability is an important component of the present study, not only because it provides beam smoothing and reproducible experimental conditions, but also because it produces a small-scale intensity distribution in the focal volume which can be described by a statistical function. A RPP is a transparent substrate with a random pattern of phase elements that introduce a phase shift of 0 or $\pi$ on the incident light. The far-field intensity distribution consists of an overall envelope determined by an individual phase plate element. Within this envelope, there is a finescale speckle structure due to the interferences between different phase element contributions, whose dimensions are determined by the $f /$ number of the focusing optics. The advantage of this technique is that it creates a welldefined intensity distribution in the laser focal spot which is nearly independent of aberrations of the initial laser beam. Within the focal region, however, there are large amplitude laser intensity fluctuations in a speckle scale length, randomly distributed, which manifest themselves in fluctuations on the SBS growth and reflectivities as discussed in this paper. The overall SBS reflectivity is the sum of contributions from the individual speckles.

The experiments have been performed using two beams of the laser facility of the Laboratoire pour l'Utilisation des Lasers Intenses (LULI) at Ecole Polytechnique. One beam, with wavelength $1.053 \mu \mathrm{m}$, was used to preform a plasma by irradiating $\mathrm{CH}$ foils $500 \mathrm{~nm}$ thick, using an $f / 8$ focusing lens. The second beam, with wavelength $\lambda_{0}=$ $0.53 \mu \mathrm{m}$, was the interaction beam, with a delay of $300 \mathrm{ps,}$ and an angle of $45^{\circ}$, with respect to the plasma forming beam. Both laser pulses have a full width at half maximum (FWHM) duration of 600 ps. The interaction beam 


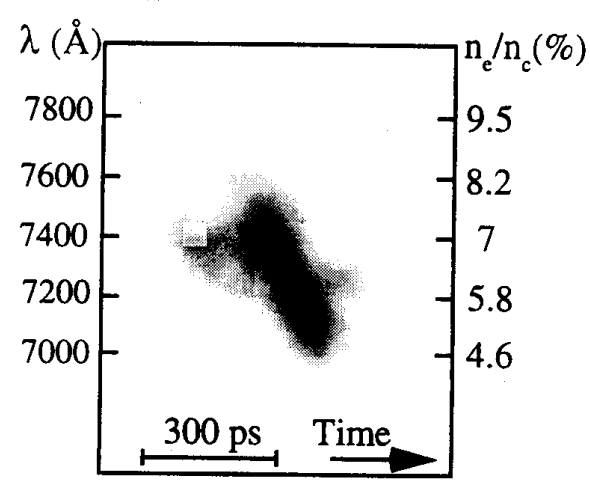

FIG. 1. Time-resolved spectrum of stimulated Raman backscattering of the interaction laser beam. Electron densities are deduced from the resonance relations of the Raman decay and the linear equations of dispersion of the waves, using an electron temperature of $0.7 \mathrm{keV}$.

was focused with an $f / 3$ lens and a RPP with $1.5 \mathrm{~mm}$ square elements. The focal spot diameter was $110 \mu \mathrm{m}$ (FWHM), with a maximum average intensity of $I_{\text {pump }}=$ $3 \times 10^{14} \mathrm{~W} / \mathrm{cm}^{2}$. The speckles generated by the RPP on the interaction beam had a mean diameter of $3 \mu \mathrm{m}$ (minimum to minimum) and a mean length of $32 \mu \mathrm{m}$. During the interaction pulse, the plasma density was below critical electron density (critical density for $\lambda_{0}=0.53 \mu \mathrm{m}$ light is $n_{c}=4 \times 10^{21} \mathrm{~cm}^{-3}$ ). The electron density profile had an approximately parabolic shape along the laser axis with a scale length of $\sim 300 \mu \mathrm{m}$, with the maximum density on axis decreasing from $0.1 n_{c}$ to $0.06 n_{c}$ during the first 300 ps of the interaction pulse. Characterization of electron density and temperature of the plasma was done using time-resolved spectra of stimulated Raman scattering in the backward direction. An example of a Raman spectrum is shown in Fig. 1. Electron density on the laser axis was deduced from the temporal evolution of the long wavelength edge of the spectra, and an electron temperature $\left(T_{e}\right)$ of $0.7 \mathrm{keV}$ was deduced from the Landau cutoff of the Raman spectra.
The diagnostic to measure the space resolved SBS consisted of a three-frame gated optical imager (GOI). This instrument yields three two-dimensional images with a

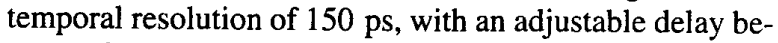
tween frames, which was chosen to be 200 ps. During the first phase of the experiment, the focal spot produced by the RPP was characterized in the absence of plasma by imaging the beam waist of the transmitted laser light. This was recorded using the same GOI instrument. During the second phase, the GOI detector assembly was installed in the back scattering diagnostic station, to measure the space distribution of the SBS emission. The spatial resolution was 5-10 $\mu \mathrm{m}$. The experimental arrangement is illustrated in Fig. 2, showing simultaneously the optical configurations to characterize the focal spot, and to image the far-field distribution of SBS in the backscattered direction. The RPP on the interaction beam was placed before the beam splitter that collected the backscattered light, to permit undisturbed imaging of SBS emission. Timeresolved SBS spectra and absolute SBS reflectivities were also measured in the backscattering direction of the interaction beam.

The laser intensity distribution at the beam waist of the focal spot in the absence of plasma is shown in Fig. 3. Since the actual location and intensities of the individual speckles vary from time to time due to fluctuations in the optical characteristics of the laser beam, a direct comparison on the location of the speckles and SBS emission is not possible (since only one GOI instrument was available), but the statistical distribution of speckles as a function of intensity will be the same during the experiment. Figure 4 shows the far-field SBS intensity distribution at three different times during the interaction pulse $(t=1.2 \mathrm{~ns}$ corresponds to the peak of the interaction beam). The data show the localized nature of the SBS reflectivity, with major contributions to the overall reflectivity coming from a few well-defined regions, located mainly in the central part of the focal spot. Some correlation in time of the location of SBS emission can be observed in the three frames. It

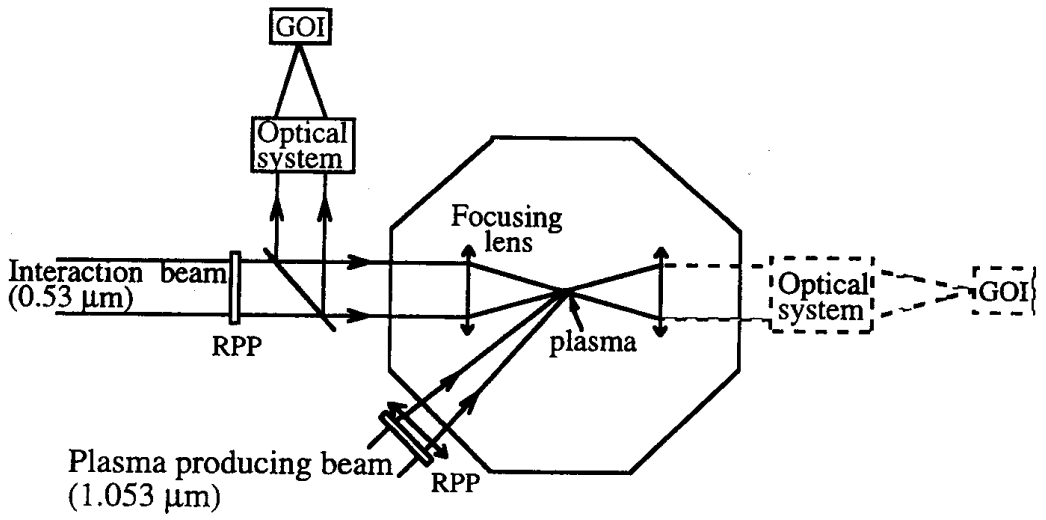

FIG. 2. Experimental arrangement for the GOI diagnostic to measure either the laser intensity distribution at best focus or the space distribution of the SBS emission. 


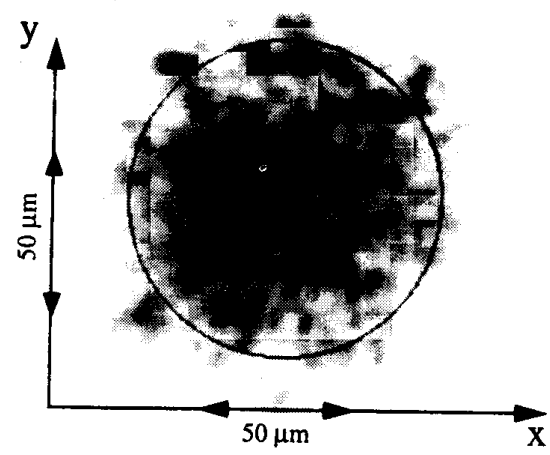

FIG. 3. Spatial laser intensity distribution at the beam waist of the focal spot in the absence of plasma. The circle has a diameter of $100 \mu \mathrm{m}$.

was observed that the SBS growth was localized in small regions and persisted for times longer than $200 \mathrm{ps,} \mathrm{but}$ shorter than the laser pulse duration.

From these images, the SBS emission was analyzed in detail to obtain the local peak reffectivities and to compare them to the total, or average in time and space, SBS reflectivity. This latter was low, typically between $10^{-4}$ and $10^{-5}$, due to plasma conditions, low laser intensities, and the use of a $0.53 \mu \mathrm{m}$ laser pump. The SBS emission from the individual spots shown in Fig. 4 was evaluated, given the local brightness of the emitting spots. For the data shown in frame 2 of Fig. 4, only $1 / 100$ of the area of the focal spot contributed to the measured reflectivity. The SBS hot spot, marked $A$ in Fig. 4, contains approximately $50 \%$ of the total reflected light, implying that the peak localized SBS emission was higher than the average reflectivity by a factor between 50 and 100 . If one takes into account the temporal evolution of the SBS emission, one ends with even higher peak reflectivities. The localized SBS emission, as indicated in Fig. 4, does not necessarily imply that these are due to single speckle contributions. Because of optical integration along the laser axis and over the optical resolution of the system, the SBS emission from one spot could be the result of the superposition of contributions from more than one speckle.

The spectrum of the backscattered SBS light was analyzed as a function of time. The time-resolved SBS spectrum, from the same shot as the GOI images of Fig. 4, is shown in Fig. 5. It is redshifted from the laser wavelength, with a shift and a broadening increasing as a function of time. Using the velocity profile from twodimensional hydrodynamics simulations, we observe that SBS starts around the isothermal sonic point, which is located $\sim 100 \mu \mathrm{m}$ in front of the summit of the density profile, and then the region of SBS emission extends towards the peak of the profile up to $\sim 100 \mu \mathrm{m}$ on the back of the target.

The SBS reflectivities, local and average, were calculated using a statistical approach $[4,6,8]$ which had been applied to the experiment using electron density, electron temperature, and velocity profiles provided by two-dimensional hydrodynamic simulations. The basic assumption of the statistical SBS theory [4] is that the scattered radiation is generated in several independent speckles, rather than homogeneously over the illuminated region of plasma. The first step of this theory is to calculate the integrated reflectivity from a single speckle, using the known SBS linear theory in stationary convective regime [7], taking into account diffraction of scattered light in the speckle and pump depletion [8]. The reflectivity of the single speckle is then averaged over the statistical distribution of speckles with different intensities in the interaction region $[6,8]$.

The SBS intensity has been calculated according to this model, and shows reasonable agreement with the experiment for the total SBS reflectivity $\left(5 \times 10^{-4}\right)$ as well as for its temporal evolution and location. The model used the profiles given by the numerical simulation, which provided good agreement with the experiment for the temporal evolution of the electron density on the laser axis as measured from the Raman spectra. More recent experiments [9] have shown improved agreement between experiment and modeling for SBS reflectivities when using

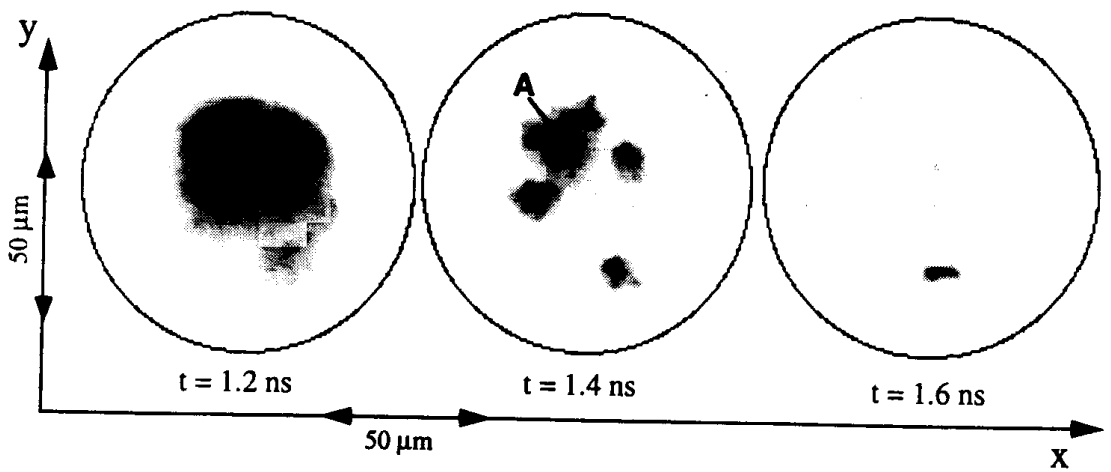

FIG. 4. Spatial intensity distribution of the SBS emission at three different times during the interaction pulse. These images are integrated over $150 \mathrm{ps} ; t=1.2 \mathrm{~ns}$ corresponds to the peak of the interaction beam. The circles are positioned in the same way as
in Fig. 3, and have a diameter of $100 \mu \mathrm{m}$. 


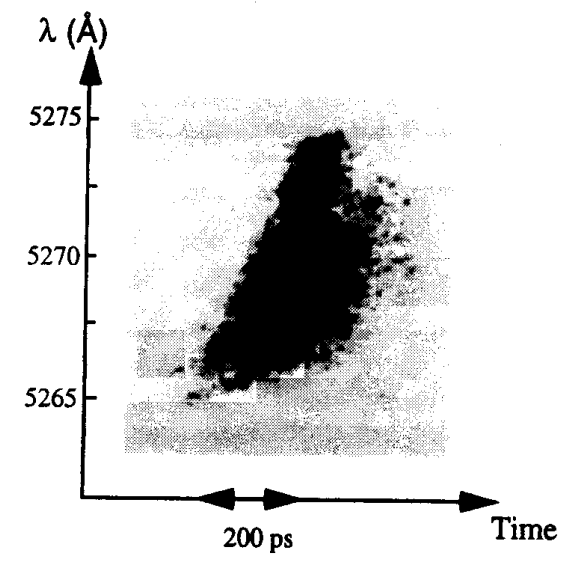

FIG. 5. Time-resolved spectrum of the SBS light collected in the focusing optics of the interaction beam. In the SBS decay, the frequency of the SBS light is given by $\omega_{\mathrm{SBS}}=\omega_{0}-\omega_{\text {iaw }}$, and the wavelength shift of the SBS light compared to the laser light is $\delta \lambda=\left(\lambda_{0}^{2} / 2 \pi c\right) k_{\mathrm{iaw}}\left(c_{s}-u\right)$, where $k_{\mathrm{iaw}}$ is the wave number of the ion acoustic wave, $c_{s}$ is the sound speed, and $u$ is the expansion velocity. The pump laser wavelength is $\lambda_{0}=5265 \AA$

the experimental shape of the electron density profile, and temperature, measured with a Thomson scattering diagnostic. At the time of SBS maximum activity, the maximum emission comes from a region $z=-20 \mu \mathrm{m}$ from the plasma center and has an extent of about $120 \mu \mathrm{m}$ FWHM. This area corresponds to the subsonic plasma expansion, in agreement with the observed redshift of the SBS spectrum.

The average SBS gain is very low for the parameters of this experiment: $\langle G\rangle=4.6 \times 10^{-16}\left[\left\langle I\left(\mathrm{~W} / \mathrm{cm}^{2}\right)\right\rangle \times\right.$ $\left.\lambda_{0}(\mu \mathrm{m}) L_{v}(\mu \mathrm{m}) n_{e} / n_{c}\right] /\left(1-n_{e} / n_{c}\right)\left[T_{e}(\mathrm{keV})+3 \times\right.$ $\left.T_{i}(\mathrm{keV}) / Z\right] \leq 1\left(L_{v}\right.$ is the velocity gradient scale length, $T_{i}$ is the ion temperature), for which the expected SBS reflectivity should be extremely small if it would not be for the presence of the statistical distribution of intensities associated with the speckled structure of the focal spot. Taking into account this gain, we can study the contribution of speckles with different intensities to the SBS reflectivity. This is a growing function of the speckle relative intensity $(u=I /\langle I\rangle)$, which ends abruptly at $u=11$ because the expected number of speckles in the interaction volume with higher intensities than this value is less than 1. Most of the SBS emission comes from speckles with intensities $u$ between 10 and 11 . If the spatial resolution of the instrument would be able to resolve one speckle, the maximum local reflectivity that would be measured will be as high as $0.15-0.2$, which is about 2000 times larger than the average SBS reflectivity. For the case of the experiment, the optical resolution $\Delta_{0}$ was larger than a speckle diameter $d_{0}$ so the maximum observed refiectivity decreases as the square of the ratio of the spatial resolution to the speckle diameter: $R_{\text {measured }}=R_{\max }\left(d_{0} / \Delta_{0}\right)^{2}$. Hence, for an instrumental resolution of about $10 \mu \mathrm{m}$, the maximum intensity will be $\sim 200$ times above the average, which is close to the experimental result.

In summary, we have presented the first detailed experimental characterization of the far-field distribution of the SBS reflectivity, showing that the emission comes from very small regions defined by the laser intensity distribution due to the RPP used in the focusing optics. Modeling of both the average and local reflectivities gives values that are very close to the experimental values, demonstrating the importance of considering the microscopic nature of the interaction. This experiment demonstrates clearly the need for detailed evaluation of the reflectivity of SBS as a function of space and time, if one is to attempt to compare experimental results with existing theoretical models. This also applies to other instabilities such as stimulated Raman scattering.

The authors gratefully acknowledge valuable discussions with Joseph Kilkenny, Kent Estabrook, and Sham Dixit, and the support of the technical groups of LULI. This work was partially supported under the auspices of the U.S. Department of Energy by the Lawrence Livermore National Laboratory under Contract No. W-7405ENG-48. Part of this support was provided through the LLNL-LDRD program under the Institute for Laser Science and Applications.

*Present address: CEA Limeil-Valenton, 94195 Villeneuve St. Georges Cedex, France.

${ }^{\dagger}$ Permanent address: P.N. Lebedev Physics Institute, Russian Academy of Science, Moscow 117924, Russia.

[1] W.L. Kruer, The Physics of Laser Plasma Interactions (Addison-Wesley, Redwood City, CA, 1988).

[2] H. A. Baldis, E. M. Campbell, and W.L. Kruer, in LaserPlasma Interactions, Handbook of Plasma Physics (NorthHolland, Amsterdam, 1991), pp. 361-434.

[3] W. L. Lindl, Phys. Plasmas 2, 3933 (1995).

[4] H.A. Rose and D.F. DuBois, Phys. Fluids B 5, 590 (1993); H. A. Rose and D. F. DuBois, Phys. Rev. Lett. 72, 2883 (1994); H. A. Rose, Phys. Plasmas 2, 2216 (1995).

[5] Y. Kato, K. Mima, N. Miyanaga, S. Arinaga, Y. Kitagawa, M. Nakatsuka, and C. Yamanaka, Phys. Rev. Lett. 53, 1057 (1984).

[6] V.T. Tikhonchuk, C. Labaune, and H. Baldis, Phys. Plasmas 3, 3777 (1996).

[7] L. M. Gorbunov and A. N. Polyanichev, Sov. Phys. JETP 47, 290 (1978); A. Ramani and C.E. Max, Phys. Fluids 26, 1079 (1983).

[8] V.T. Tikhonchuk, D. Pesme, and Ph. Mounaix, Phys. Plasmas 4, 2658 (1997).

[9] C. Labaune, H. Baldis, and V.T. Tikhonchuk, Europhys. Lett. 38, 31 (1997). 
Time-resolved measurements of secondary Langmuir waves produced by the Langmuir decay instability in a laser-produced plasma

\author{
C. Labaune, H. A. Baidis, ${ }^{\text {a) }}$ and B. S. Bauer) \\ Laboratoire pour l'Utilisation des Lasers Intenses, Ecole Polytechnique, Centre National de la Recherche \\ Scientifique, 91128 Palaiseau cedex, France \\ V. T. Tikhonchuk ${ }^{\mathrm{c})}$ and G. Laval \\ Centre de Physique Théorique, Ecole Polytechnique, Centre National de la Recherche Scientifique. \\ 91128 Palaiseau cedex, France
}

(Received 13 May 1997; accepted 30 September 1997)

\begin{abstract}
Direct observations of secondary Langmuir waves produced by the parametric decay instability of primary Langmuir waves are presented. The measurements have been obtained using Thomson scattering of a short-wavelength probe laser beam and are resolved in time, space, frequency, and wave number. The primary Langmuir waves were driven by stimulated Raman scattering (SRS) of a smoothed laser beam in a preformed plasma. Measurements of the amplitude of the density fluctuations associated with primary and secondary Langmuir waves show that the threshold of the Langmuir decay instability (LDI) is close to the threshold of the Raman instability. This is in agreement with theoretical predictions. However, the ratio of amplitudes of the density fluctuations associated with both secondary and primary Langmuir waves does not agree with existing theories of SRS saturation due to LDI cascading and/or strong Langmuir turbulence in homogeneous plasmas. An explanation based on the interaction beam intensity distribution produced by the random phase plate in the plasma is discussed. (c) 1998 American Institute of Physics.
\end{abstract} [S1070-664X(98)02101-6]

\section{INTRODUCTION}

Langmuir waves, or electron plasma waves (EPW), can be easily excited in a plasma and are responsible for a variety of nonlinear effects in many plasma applications, like inertial confinement fusion, particle acceleration, current drive, and microwave heating in tokamaks, $x$-ray lasers, and iono-. spheric plasma modification. ${ }^{1}$ EPWs are especially important in laser plasmas as encountered in the context of inertial confinement fusion by laser beams, ${ }^{2}$ as they can accelerate electrons to high energy that preheats the fusion fuel and reduces the target gain. They can also scatter large amounts of incident laser light in undesired directions. Several mechanisms have been identified that can generate EPWs in laserproduced plasmas: stimulated Raman scattering (SRS), twoplasmon decay, parametric decay instability, Langmuir decay instability, resonance absorption, and hot electron pulses. ${ }^{3}$ Because of weak dissipation of these EPWs due to Landau or collisional damping, Langmuir waves interact between themselves, couple to ion acoustic waves, and often bring plasma into a turbulent state. ${ }^{4-6}$ Previous laser-plasma interaction experiments have focused on qualitative spectral indications of the mechanisms of EPW generation, coupling, and dissipation, while there was no direct measurement of EPW decays. EPWs are difficult to diagnose as they do not radiate directly light outside the plasma. Scattering of an

\footnotetext{
al Also at the Institute for Laser Science and Applications (ILSA), Lawrence Livermore National Laboratory, P.O. Box 808, Livermore California 94550.

${ }^{b)}$ Present address: Department of Physics, University of Nevada, Reno, Nevada 89557-0058.

c'Present address: P. N. Lebedev Physics Institute, Russian Academy of Science, Moscow 117924, Russia.
}

electromagnetic wave off EPWS is a common way to collect and analyze light associated with EPWs. The most frequently used diagnostic of parametric instabilities had been based on the scattering of the incident laser light itself from the longitudinal waves resulting from the decay. ${ }^{7}$ However, information on longitudinal waves can be distorted by the propagation and nonlinear interaction of the electromagnetic wave in a dense plasma. Thomson scattering of a short-wavelength and low-intensity laser beam ${ }^{8}$ is a much more relevant method to measure directly the spectrum of density fluctuations in a plasma, and identify waves associated with specific decays.

In this paper we describe results of an experiment that was conducted to study features of the Langmuir decay instability, including threshold, growth, and temporal evolution of the plasma waves. The Langmuir decay instability (LDI) is the decay of a primary Langmuir wave into a secondary Langmuir wave and an ion acoustic wave. First theoretical predictions of this instability were reported by DuBois and Goldman. ${ }^{6}$ Only indirect experimental indications of LDI have been reported so far, based on the dependence of SRS reflectivity on EPW damping or broadening of SRS spectra. ${ }^{9.10}$ In the present experiment, SRS was used as the source of EPWs, as the Langmuir wave spectrum driven by SRS is simpler than the primary spectrum, driven by either the ion acoustic decay instability or the two-plasmon decay. In particular, EPWs driven by SRS are emitted only in the forward direction of the laser propagation. Thus, any EPWs traveling antiparallel to the laser wave vector must be due to the LDI or another mechanism that can reverse the EPW's wave vector. LDI is of particular importance for SRS in the context of laser-driven inertial confinement fusion, as it had been proposed as one of the possible mechanisms respon- 
sible for the saturation of the instability. ${ }^{11-14}$ In the experiment presented in this paper, both primary and secondary EPWs, coming from the Langmuir decay of the first ones, have been observed on the same time-resolved spectra of the Thomson scattered light. The threshold of the Langmuir decay instability has been observed to be close to the threshold of the Raman instability, as predicted by theory. However, the maximum ratio of the amplitudes of secondary and primary EPW was found to be less than what was expected from theories of SRS saturation due to LDI cascading ${ }^{11.12,14}$ and/or strong Langmuir turbulence ${ }^{13}$ in homogeneous plasmas.

The Langmuir decay instability (LDI) is a three-wave process, where the dispersion relation is satisfied for each wave, as well as conservation of energy and momentum: $\omega_{\text {epw1 }}=\omega_{\text {epw } 2}+\omega_{\text {iaw } 2}$ and $\mathbf{k}_{\text {epw } 1}=\mathbf{k}_{\text {epw } 2}+\mathbf{k}_{\mathrm{iaw} 2}$, where $\omega_{i}$ and $\mathbf{k}_{i}$ are the frequencies and wave numbers of the pump (epw1), the daughter or secondary Langmuir waves (epw2), and the daughter ion acoustic wave (iaw2). In this paper we report the study of the backward decay, which is the most probable, for which the secondary EPW is emitted in backward direction compared to the primary one. Taking into account that the frequency of the ion acoustic wave is much smaller than the frequency of the Langmuir waves, the frequencies and wave numbers of both EPWs are almost identical but propagating in opposite directions. The plasma parameters have been chosen so that the maximum electron density was below quarter-critical for most of the interaction puise, to eliminate sources of EPWs associated with mechanisms at critical density (resonance absorption or parametric decay instability), or at quarter-critical density (two-plasmon decay), thus limiting the types of EPWs present in the plasma.

The experimental setup, beam configuration, plasma characteristics, and diagnostics are described in Sec. II. Experimental results on threshold, growth, and temporal evolution of EPWs associated both with SRS and LDI, are presented in Sec. III. Other possible mechanisms of production of the observed counterpropagating EPWs besides LDI are discussed in Sec. IV. All these other mechanisms are ruled out, leaving LDI as the only candidate to explain the observed EPWs. In Sec. $V$ we present theoretical results on LDI and their comparison with experiments. Interpretation of SRS and LDI is based on the speckles distribution of the laser intensity in the focal volume produced by the random phase plate placed on the interaction beam. In Sec. VI we conclude this paper.

\section{EXPERIMENTAL CONDITIONS AND DIAGNOSTICS}

The experiments have been performed with the six-beam laser facility of the Laboratoire pour l'Utilisation des Lasers Intenses (LULI), using a $1.053 \mu \mathrm{m}$ interaction beam and a well-characterized preformed plasma. The laser beam configuration and the principal diagnostic of this experiment are shown in Fig. 1. The 600 ps full-width half-maximum (FWHM) Gaussian beams were all in the same plane and arrived at the target plane at different times. Two beams, converted to the second harmonic $(\lambda=527 \mathrm{~nm})$, arrived at $t=0$ from opposite directions on thin plastic disks $(\mathrm{CH})_{n}$ to

Phys. Plasmas, Vol. 5, No. 1, January 1998

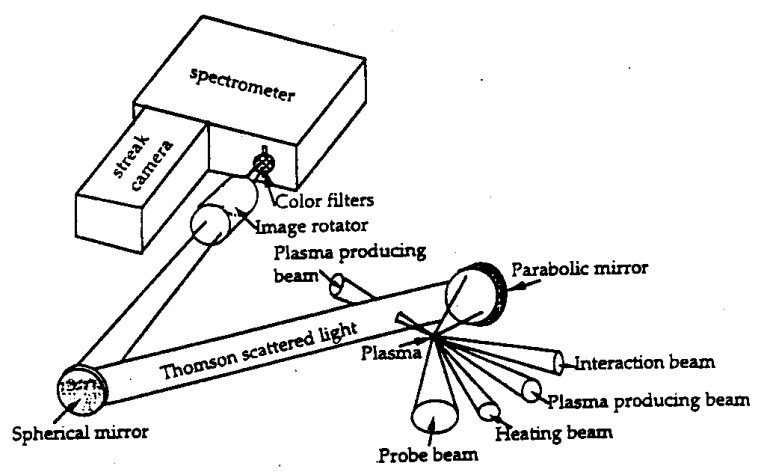

FIG. 1. Experimental setup showing the beam configuration and the main diagnostic based on time-resolved spectra of Thomson scattered light of a short-wavelength probe beam.

produce the plasma. The initial foil thickness, 0.94 or $1.2 \mu \mathrm{m}$ (altematively), was chosen for the targets to bum through toward the end of the primary plasma producing beams. At $t=1.1 \mathrm{~ns}$, a third $0.53 \mu \mathrm{m}$ laser beam was used to heat the plasma. Random phase plates (RPP) were used on the beams producing and heating the plasma to obtain plasma conditions as reproducible as possible. The $1.053 \mu \mathrm{m}$ interaction beam was focused with an $f / 6$ lens through a RPP of almost square $2 \mathrm{~mm}$ elements, after a delay of $2 \mathrm{~ns}$ with respect to the plasma-forming beams. The combination of the focusing lens and the RPP produced an Airy pattern central maximum with $65 \%$ of the total energy in a spot diameter of $320 \mu \mathrm{m}$. Within this spot, the maximum average intensity was $10^{14} \mathrm{~W} / \mathrm{cm}^{2}$, for an energy of $100 \mathrm{~J}$. The interaction beam energy was varied from 20 to $85 \mathrm{~J}$, above the observational threshold for SRS. The mean FWHM diameter of the speckles was $9 \mu \mathrm{m}$, and their mean length was $250 \mu \mathrm{m}$.

The target orientation was chosen perpendicular to the interaction beam in order to present an axially symmetric long-scale-length plasma to the interaction beam. Electron density was measured, as a function of position along the laser beam axis and time, using time-resolved spectra of the scattered light from electron plasma waves, associated with SRS, recorded simultaneously at different places in the plasma, ${ }^{15}$ by using multiple slits on the streak camera. ${ }^{16}$ The density profile had an approximately inverse parabolic shape along the laser axis with a scale length of $\sim 1 \mathrm{~mm}$. For the $1.2 \mu \mathrm{m}$ targets, the maximum electron density on the axis decreased from $0.25 n_{c}$ to $0.05 n_{c}$ during the interaction pulse due to plasma expansion ( $n_{c}$ is the critical density for 1.053 $\mu \mathrm{m}$ laser light, $\left.n_{c}=1.1 \times 10^{21} \mathrm{~cm}^{-3}\right)$. The electron temperature measured using thermal Thomson scattering in the absence of interaction beam ${ }^{17}$ was $\sim 0.5 \mathrm{keV}$ at $t=1.7 \mathrm{~ns}$ at the center of the plasma. Two-dimensional hydrodynamics simulations show that the plasma was heated due to the interaction beam to $\sim 0.7 \mathrm{keV}$ at the peak of the interaction pulse, and then decreased due to plasma cooling because of expansion.

The EPWs were observed along the interaction beam axis using collective Thomson scattering (CTS) of a $351 \mathrm{~nm}$ wavelength, 600 ps Gaussian, probe beam that started 240 ps before the interaction pulse. The probe beam was focused 


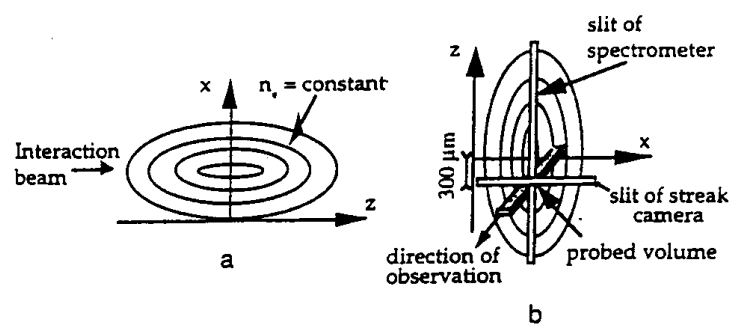

FIG. 2. Imaging of the plasma to the entrance slit of the spectrometer. (a) Sketch of the plasma electron isodensities $\left(n_{e}=\right.$ const), indicating the direction of the interaction laser beam. (b) Image of the plasma at the entrance slit of the spectrometer, after rotation by $90^{\circ}$. The probed volume is located $300 \mu \mathrm{m}$ in the front part of the plasma. It is $75 \mu \mathrm{m}$ along the interaction axis, $70 \mu \mathrm{m}$ in height, and $300 \mu \mathrm{m}$ along the transverse direction.

onto the plasma with an $f / 3$ lens combined with a RPP with elongated elements $(0.1 \times 0.9) \mathrm{mm}^{2}$, producing a line focus of $100 \mu \mathrm{m} \times 1 \mathrm{~mm}$ along the axis of the interaction beam. The Thomson scattered light was collected with an off-axis, $34^{\circ}$ aperture parabolic mirror, which was part of the optical system imaging the focal region onto the entrance slit of a spectrometer, after a rotation by $90^{\circ}$. The output of the spectrometer was coupled to a streak camera. The CTS spectrometer-streak camera combination was aligned to observe scattered light from a small volume in the region of peak SRS-driven EPW activity, which was $\sim 300 \mu \mathrm{m}$ toward the interaction beam from the plasma center. ${ }^{18}$ The size of the probed volume was set by the magnification of the imaging system, the width of the slits of the spectrometer, and the streak camera, and the focal spot diameter of the interaction beam. This was $75 \mu \mathrm{m}$ along the interaction axis, $70 \mu \mathrm{m}$ in height, and $300 \mu \mathrm{m}$ along the transverse direction. A scheme of the probed volume along with isodensities of the plasma is shown in Fig. 2. The range of wavelengths observed was between 280 and $480 \mathrm{~nm}$, with a temporal resolution of 50 ps and a spectral resolution of $30 \AA$. Timeintegrated results, obtained under different pumping conditions and with different diagnostics, have been previously reported. $^{19}$

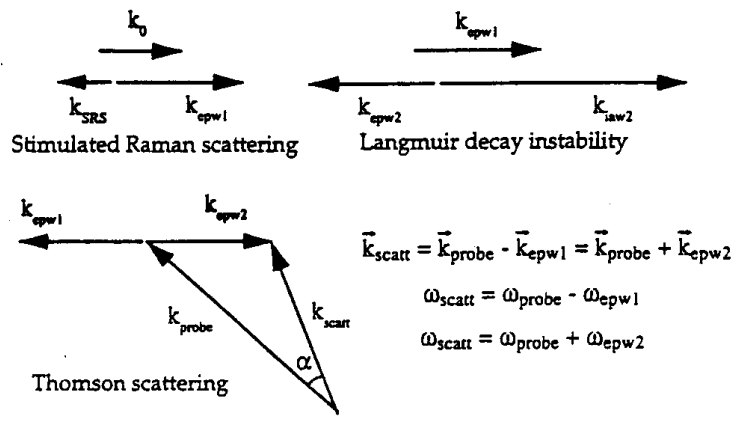

FIG. 3. Wave-vector diagrams for the SRS of the interaction beam, the LDI of the electron plasma waves produced by SRS, and Thomson scattering of the probe beam. The relationships between the wave numbers and frequencies of the probe beam $\left(k_{\text {probe }}, \omega_{\text {probe }}\right)$, the EPW $\left(k_{\text {epw 1.2 }}, \omega_{\text {epwi.2 }}\right)$, and scattering beam $\left(k_{\text {sean }}, \omega_{\text {seat }}\right)$ are also indicated.
The geometry of the CTS diagnostic was designed to collect downshifted scattered light $\left(\omega_{\text {down }}=\omega_{\text {probe }}-\omega_{\text {cpwl }}\right)$ from the primary EPWs associated with backward SRS. Upshifted scattered light $\left(\omega_{\text {up }}=\omega_{\text {probe }}+\omega_{\text {epw } 2}\right.$ ) from secondary EPWs having same frequency and opposite wave numbers as the primary EPWs was emitted in the same direction and collected by the same CTS detector optics. Figure 3 shows the general wave vector diagrams for the Raman and the LDI decays, and for Thomson scattering, as well as the relationships between the wave numbers and frequencies of the probe beam $\left(k_{\text {probe }}, \omega_{\text {probe }}\right)$, the EPW $\left(k_{\text {epwl,2 }}, \omega_{\text {epw 1,2 }}\right)$ and scattered beam $\left(k_{\text {scan }}, \omega_{\text {scart }}\right)$. To ensure that the two strongly different light amplitudes both fell within the streak camera dynamic range, colored filters that attenuated only the SRSdriven EPW scattered light were placed before the spectrometer and a neutral filter was positioned over only the SRSdriven EPW side of the spectrum, after the spectrometer, at the streak camera entrance slit. The spectral dependence of the total optical system was taken into account when unfolding the data to make quantitative wave-amplitude comparisons.
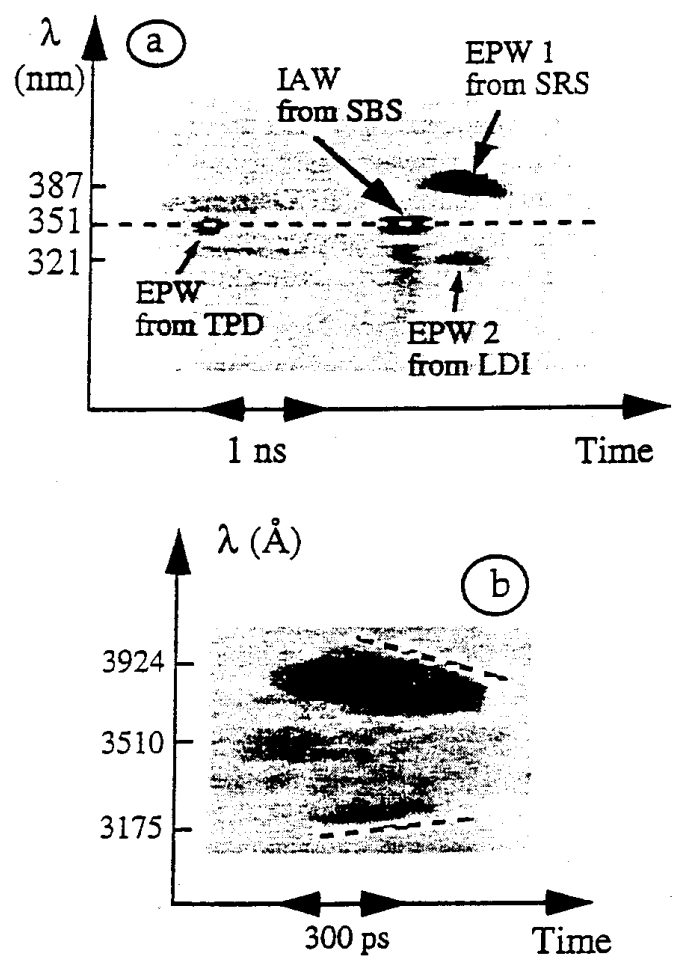

FIG. 4. (a) Time-resolved spectra of the Thomson scattered light from ion acoustic waves associated with stimulated Brillouin scattering, primary elec tron plasma waves associated with stimulated Raman scattering, and secondary electron plasma waves associated with the Langmuir decay of the former ones. These three types of waves are driven by the interaction beam. The first signal in time is Thomson scattered light from electron plasma waves produced by the two-plasmon decay of the plasma-producing beams. Intensity of the interaction beam was $8 \times 10^{13} \mathrm{~W} / \mathrm{cm}^{2}$. Different optical attenuations have been used for the four signals. (b) Temporal evolution of scattered light from primary and secondary EPWs showing symmetrical slopes in frequencies as a function of time $\left[d \omega_{\text {up }} / d t / d \omega_{\text {down }} / d t\right.$ $\left.=\left(\lambda_{\text {down }} / \lambda_{\text {up }}\right)^{2} d \lambda_{\text {up }} / d t / d \lambda_{\text {unwn }} / d t=-1\right]$. 


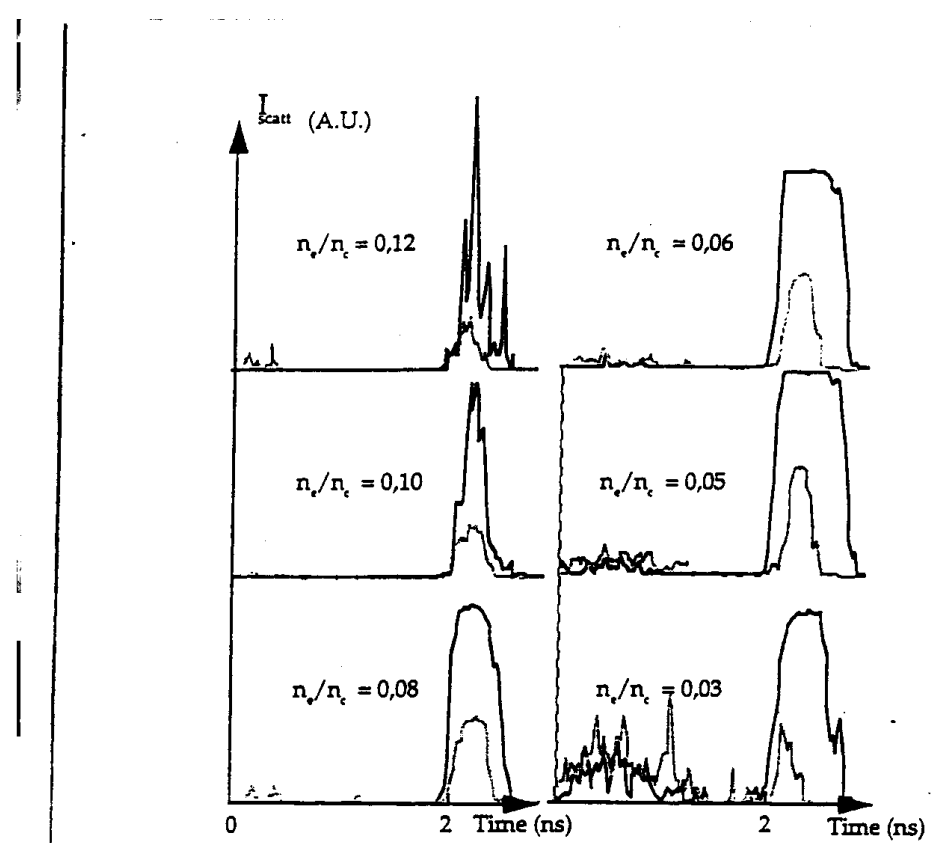

FIG. 5. Temporal evolution of scattered intensities from primary (in black) and secondary (in grey) EPWs for specific electron densities between $12 \%$ and $3 \%$ of critical density. Different attenuations were used for the up- and downshifted, and for different electron densities.

\section{TIME-RESOLVED THOMSON SCATTERING SPECTRA}

Two typical time-resolved spectra of CTS light are shown in Fig. 4. Time runs from left to right and wavelength from bottom to top. The earliest signal observed in Fig. 4(a) at $351 \mathrm{~nm}$ comes from 3/2-harmonic emission of the $526 \mathrm{~nm}$ plasma-producing beams, caused by the two-plasmon decay (TPD). Another burst of $351 \mathrm{~nm}$ light follows $1.85 \mathrm{~ns}$ later, early in the interaction pulse. This is light scattered by. ion acoustic waves (LAW) associated with stimulated Brillouin scattering (SBS). About $30 \mathrm{~nm}$ above this burst (red-shifted), and 0.24 ns later, is the scattered light from the primary EPWS driven by SRS of the interaction beam, and simultaneous with it, symmetrically below the $351 \mathrm{~nm}$ probe frequency, is the blue-shifted light from the secondary EPWs. The optical attenuations used in front of the spectrometer are frequency dependent, so the apparent intensities observed in this figure are not significant of the relative amplitudes of the waves. The edge of the neutral density Wratten filter (attenuation by a factor of 10 ), placed at the streak camera entrance slit, can be seen by the apparent sharp change in plasma light intensity at $340 \mathrm{~nm}$ (blue-shifted light below $340 \mathrm{~nm}$ is unattenuated).

Plasma densities, at which both primary and secondary EPWs have been produced, can be deduced from the scattered wavelengths using the following relation: $n_{e} / n_{c}=9$ (1 $\left.-\lambda_{\text {probe }} / \lambda_{\text {TS }}\right)^{2}$. This was obtained by neglecting the BohmGross correction to the EPW frequency, which would give a correction $\leqslant 5 \%$ for the electron temperature encountered in these experiments. The spectra of Fig. 4 correspond to maximum plasma densities evolving between $12 \%$ and $3 \%$ of the critical density, for both EPW components. We observe exact synchronization and symmetry of the EPW's frequencies with respect to the probe frequency as a function of time. At any given time the width of the EPW spectra can be interpreted as due to the range of plasma densities that the probe beam encounters along the transverse direction of the interaction laser. The higher cutoff for the downshifted component and a lower cutoff for the upshifted one, correspond to the highest plasma densities that are on the laser axis. The change of frequency as a function of time is due to the decrease of the electron density due to plasma expansion, and we observe symmetrical slopes in frequencies ( $d \omega_{\text {upscart }} / d t$ $=-d \omega_{\text {downscant }} / d t$ ), as shown in Fig. $4(\mathrm{~b})$.

Time evolution of the scattered intensities from primary and secondary EPWs is shown in Fig. 5 for specific electron density between $12 \%$ and $3 \%$ of critical density. This demonstrates a perfect correlation in time between the two types

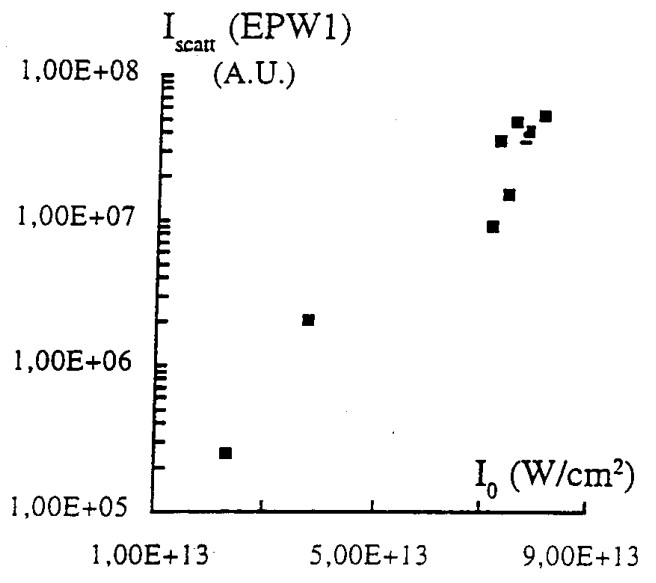

(a)

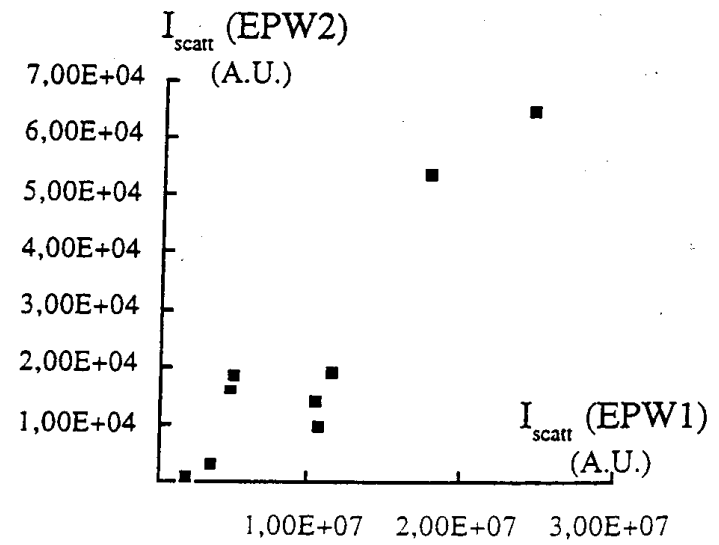

(b)

FG. 6. (a) Variation of total intensity of scattered light from electron plasma waves created by stimulated Raman backscattering as a function of the laser pump intensity. (b) The relation between intensities of secondary and primary EPWs. 


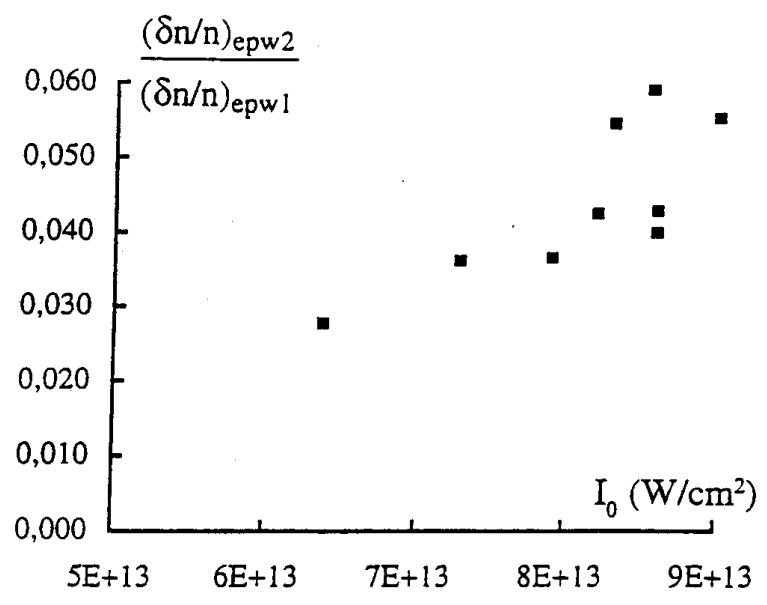

FIG. 7. The ratio of the amplitudes of the density fluctuations associated with secondary and primary EPWs as a function of the pump intensity.

of waves. Different attenuations are used for the up- and downshifted, and for different electron densities. For the highest densities, $n_{e} / n_{c} \geqslant 10 \%$, the two signals have exactly the same duration. At low density, $n_{e} / n_{c} \leqslant 8 \%$, the upshifted signal is shorter in time and peaks in advance of the downshifted one. This is particularly significant at $n_{e} / n_{c}=3 \%$, where the upshifted signal is tumed off when the intensity of the downshifted signal is still large.

The measured thresholds for SRS and LDI were 2 $\times 10^{13}$ and $5 \times 10^{13} \mathrm{~W} / \mathrm{cm}^{2}$, respectively. Above these thresholds, scattered intensities from both EPWs increase fast with the pump intensity, as shown in Fig. 6. The scattered intensity associated with the primary EPWs [Fig. 6(a)] increases by more than two orders of magnitude when the pump intensity is increased by a factor of $\sim 4$, with an approximately exponential law, $I_{\text {scatt-epw } 1} \sim \exp \left(2 G_{R}\right)$. Assuming that the SRS gain $G_{R}$ exp is proportional to the laser intensity and approximating the experimental points in Fig. 6 with a straight line, one finds that $G_{R}$ exp varies from 2 for $I=3 \times 10^{13} \mathrm{~W} / \mathrm{cm}^{2}$ to 4 for $I=9 \times 10^{13} \mathrm{~W} / \mathrm{cm}^{2}$. Figure $6(\mathrm{~b})$ shows the dependency of the scattered intensity from secondary EPWs as a function of the scattered intensity from primary EPWs, exhibiting a threshold for the apparition of the counterpropagating EPWs.

The measured scattered power corresponds to a maximum average density fluctuations level of $\delta n / n \sim 10^{-3}$ for the primary EPWs associated with backscattered SRS. ${ }^{8}$ From the CTS spectra, integrated over time and wavelengths, we have measured the ratio of the amplitudes of the density fluctuations associated with the primary and secondary EPWs. This ratio is plotted as a function of the pump intensity in Fig. 7. It varies between 37 for the pump intensity very close to threshold of LDI, to 17 for the highest intensity used in this experiment. As can be seen in Fig. 5, this ratio does not depend much on time, nor on electron density.

\section{ORIGIN OF THE COUNTERPROPAGATING EPWS}

Apart from LDI, other processes have been considered as possible sources for the observed counterpropagating
EPWs, and they have been ruled out for reasons that will be discussed in this section. As the primary EPWs are created in the front part of the plasma, they travel in the direction of increasing densities, and could be reflected at their critical density. The distance between the SRS resonance point, where the primary EPWs are created, and their turning point is $\Delta z \approx 3\left(k_{\text {epw } \mid \lambda_{D}}\right)^{2} L_{n}$, where $\lambda_{D}$ is the Debye length and $L_{n}$ is the density gradient scale length. For typical parameters of the experiment, $k_{\mathrm{epw}} \lambda_{\mathrm{D}} \approx 0.15$ at $n_{e} / n_{c}=0.1$, and $L_{n}$ $=1 \mathrm{~mm}$, we find $\Delta z \approx 70 \mu \mathrm{m}$. The damping length of the EPWs can be estimated from their group velocity $v_{g \text { epw }}$ and their damping rate $\nu_{\text {epw }}: 1_{\text {damping }} \approx v_{g \text { epw }} / \nu_{\text {epw }}$ $=3 k_{\text {epw }} \lambda_{D}^{2} \omega_{\text {epw }} / \nu_{\text {epw }}$. The EPW damping is mainly collisional for densities higher than $0.05 n_{c}$ and collisionless for densities lower than $0.05 n_{c}$, yielding for $T_{e}=0.5 \mathrm{keV}$ and EPW damping length of $8 \mu \mathrm{m}$ at $n_{e} / n_{c}=0.1$ and $2 \mu \mathrm{m}$ at $n_{e} / n_{\varepsilon}=0.04$. Even if including some uncertainty on the local value of $L_{n}$, these numbers rule out the possibility for the primary EPWs to propagate to their tuming point and retum to the region of observation, taking into account the wide range of densities where the secondary EPWs were observed.

Reflection of primary EPWs from ion density fluctuations could be another mechanism for the secondary EPW generation. This requires large-density perturbation amplitudes $(\sim 10 \%)$ that have not been observed in this experiment and that would likely quench the SRS itself due to additional EPW damping. ${ }^{20}$

The generation of secondary Langmuir waves due to mode coupling between primary EPW and IAW associated with $\mathrm{SBS}^{21}$ has been ruled out for two reasons. The first one is the observed delay in time between the two instabilities, as shown in Fig. 4, where the overiap between the two types of waves is very short. ${ }^{22}$ SBS starts first, early in the interaction pulse, and SRS starts to grow only when SBS is disappearing. The second reason is that the wave number of EPWs produced by such a mechanism would not match the CTS collecting direction. It would be $k_{\text {epw } m}=k_{\text {epw } 1}+m k_{\text {iaw }}$, with $k_{\text {iaw }} \approx 2 k_{0}$ and $m$ being any positive or negative number. The closest $m$ is $m=-2$, which gives, for the density region $n_{e} / n_{c}=0.03$ to $0.1, k_{\text {epw }}=-2.2 k_{0}$ to $-2.4 k_{0}$, where $k_{0}$ is the wave number of the interaction wave. This is different from the wave number of the CTS observed EPW, which is between 1.2 and $1.8 k_{0}$, and the light scattered out of such waves would be out of the collecting optics.

The last mechanism that can be considered as a possible source of Langmuir waves traveling in an opposite direction to the incident laser is the excitation of EPWs by a stream of fast electrons. ${ }^{23}$ The hot electrons will have to have a velocity equal to the EPW phase velocity. This corresponds to electron energy $>20 \mathrm{keV}$, which are unlikely to be present in this experiment because of the lack of critical or quartercritical densities, where they might be generated. In addition, the spectrum of Langmuir waves produced by this process would be much broader than what was observed in the experiment.

\section{THE LANGMUIR DECAY INSTABILITY}

In the previous section we have shown that the Langmuir decay instability is the only candidate to explain the 


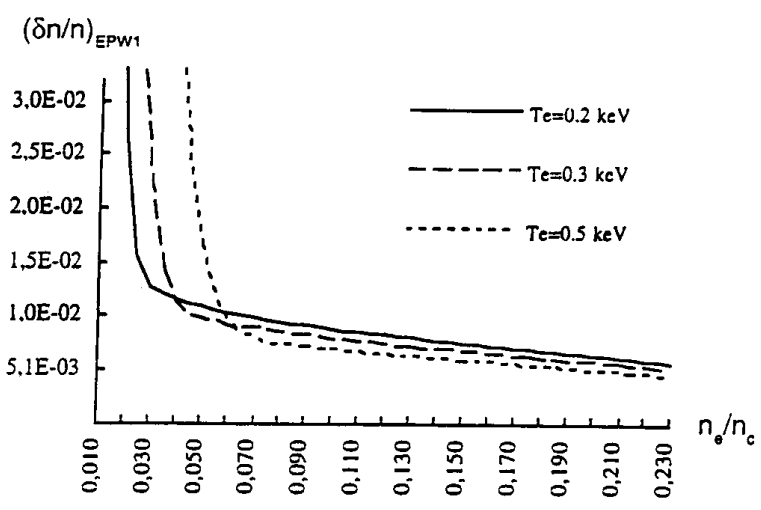

FIG. 8. Density fluctuations of the primary EPWs, $(\delta n / n)_{\text {epwl }}$, required to reach the threshold of the Langmuir decay instability as a function of electron density of the piasma, and for three electron temperatures.

observed secondary EPWs in this experiment. The necessary condition for this instability to grow is that the amplitude of the primary EPW exceeds the damping threshold: ${ }^{6}$

$$
\left(\frac{\delta n}{n}\right)_{\text {epw1 }}=4 k_{\text {epw1 }} \lambda_{\mathrm{D}} \sqrt{\frac{\nu_{\mathrm{ia} 2}}{\omega_{i a 2}} \frac{\nu_{\mathrm{epw} 2}}{\omega_{\mathrm{epw} 2}}},
$$

where $\nu_{\text {iaw2.epw2 }}$, are the damping rates of the secondary IAW and EPW, respectively. For our plasma parameters, with $Z T_{e} / T_{i} \sim 8$, which correspond to a moderate IAW damping in $\mathrm{CH}$ plasmas, $\nu_{\mathrm{i} 2} / \omega_{\mathrm{ia} 2} \approx 0.1,{ }^{24}$ the threshold density fluctuations $(\delta n / n)_{\text {epwl }}$ is plotted as a function of electron density in Fig. 8, for three electron temperatures, $T_{e}$ $=0.2,0.3$, and $0.5 \mathrm{keV}$. For densities larger than $7 \%$ of critical density, the LDI threshold corresponds to $(\delta n / n)_{\text {epwl }}$ $\sim 7 \times 10^{-3}$; the threshold increases rapidly at low electron densities. Density fluctuations of $7 \times 10^{-3}$ are larger than the measured average amplitude of SRS-EPWs, and the strong increase of threshold for densities lower than $5 \%$ of critical at $0.5 \mathrm{keV}$ would not let LDI to occur at $n_{e} / n_{c}=3 \%$, as it had been observed. This first argument demonstrates that we cannot explain the experimental results in terms of average quantities. One can notice that this theoretical threshold applies to a monochromatic Langmuir wave pump. For a broadband SRS-driven EPW, the LDI threshold might be higher, which does not affect our previous statement.

The same problem arises with the convective SRS gain calculated for average laser intensity. Assuming convective SRS in an inhomogeneous density profile, the exponential amplification of EPWs associated with SRS can be calculated using the convective SRS gain coefficient:

$$
G_{R \mathrm{th}}=1.8 \times 10^{-18}\left(I \lambda^{2}\right)\left(\frac{L_{n}}{\lambda}\right) \frac{\left(k_{\mathrm{epw1}}\right)^{2}}{k_{\mathrm{SRS}} k_{0}},
$$

where $I$ is the pump intensity in $W / \mathrm{cm}^{2}, \lambda$ is the laser wavelength in $\mu \mathrm{m}, L_{n}$ is the density gradient scale length in $\mu \mathrm{m}$, $k_{\text {epw } 1}$ and $k_{\text {SRS }}$ are the wave numbers of the EPWI and scattered Raman light, respectively. For our conditions, $I=9$ $\times 10^{13} \mathrm{~W} / \mathrm{cm}^{2}, L_{n}=1 \mathrm{~mm}, n_{e} / n_{c}=0.08$, we get: $G_{R \mathrm{th}}=0.7$. This value is too small to explain the observed primary EPW's amplitude, and no secondary EPWs would have been expected. This is aiso in contradiction with the SRS gain, which was estimated from Fig. 6: $G_{R}$ exp $\approx 3.5$ for the same laser intensity.

These two apparent contradictions can be resolved if we account for the inhomogeneous intensity distribution of the interaction beam produced by the random phase plate. Previous calculations assumed an homogeneous laser intensity distribution, and it has been demonstrated analytically ${ }^{25}$ and numerically ${ }^{26}$ that SRS from randomized laser beams has a lower threshold than perfect beams. In the focal volume, the laser intensity is distributed over hot spots or speckles, some of them having a much higher intensity than the average. These high-intensity speckles are responsible for most of the SRS signals. We define the parameter $u$ as the ratio of the maximum intensity $I$ of a speckle to the average intensity $\langle I\rangle: u=I /\langle I\rangle$. The statistical distribution of these speckles in a plasma can be described by the three-dimensional probability function $M_{3}(u) \sim\left(V_{f} / V_{\mathrm{sp}}\right) u e^{-u},{ }^{25}$ where $V_{f}$ is the focal volume and $V_{\text {sp }}$ is the characteristic volume of a single speckle. From the above comparison between the calculated and measured SRS gains, we conclude that SRS cannot grow in the overall focal volume and must occur in speckles with higher intensities than average. This hypothesis is reminiscent of the idea of Ref. 27 that SRS emission cannot be explained with average laser intensity, although that paper was based on SRS emission from filaments, and no RPP was used in the experiment. This idea is also in agreement with our previous modeling of stimulated Brillouin scattering (SBS) growing in speckles, which led to good agreement with the experimental results on SBS reflectivities and ion acoustic fluctuation levels. ${ }^{28}$ The theory of SRS from RPP laser beams has been derived in Ref. 25 . Corresponding numerical simulations are described in Ref. 26.

According to the measured SRS gain, speckles with $u$ $\geqslant 5$, having a convective SRS gain of the order of 3.5 , could explain the observed SRS gain and EPW's amplitude. For our parameters, $V_{f} \approx 810^{7} \mu \mathrm{m}^{3}$ and $V_{\mathrm{sp}} \approx 1.6 \times 10^{4} \mu \mathrm{m}^{3}$, the expected number of such speckles, $M_{3}(5)$, in the focal volume is around 170, which is large enough to ensure the statistical approach. A more accurate way to estimate the effective SRS gain is following. According to the statistical theory, ${ }^{25}$ the EPW intensity and the intensity of the scattered light scale as $I_{\text {scatt }} \sim \exp \left[\left(2 G_{R}-1\right) u_{\max }\right]$, where $u_{\max }$ is the maximum intensity of the speckle that can be found in the scattering volume. We can estimate $u_{\max } \sim 7$ from the condition that the expectancy to find higher-intensity speckles is small: $M\left(u_{\max }\right) \sim 1$. Then the effective SRS gain, $G_{\text {ef }}=\left(G_{R}\right.$ $-1 / 2) u_{\max }$, will be about 1.4 for $I=9 \times 10^{13} \mathrm{~W} / \mathrm{cm}^{2}$, which is smaller than the experimental value. Probably, this difference could be attributed, at least partially, to self-focusing of laser light in speckles. Indeed, the laser power in a speckle with $u=5$ for the average intensity $I=9 \times 10^{13} \mathrm{~W} / \mathrm{cm}^{2}$ is about $200 \mathrm{MW}$, which is comparable to the critical power for self-focusing for our plasma parameters. It has been shown in Ref. 29 that the effect of self-focusing will be to decrease the apparent threshold of the instability. If we take 0.2 , instead of 0.5 , for the threshold in the formula of $G_{e f}$, we then obtain a gain of 3.5 , in agreement with the experimental value. Self-focusing will also explain the discrepancy on the 
SRS threshold between theory and experiment. The SRS threshold predicted by the statistical theory for $G_{R}=1 / 2$ corresponds to a laser intensity of $6 \times 10^{13} \mathrm{~W} / \mathrm{cm}^{2}$, which is three times above the measured value. Using $G_{R}=0.2$ lowers the theoretical threshold to $2.4 \times 10^{13} \mathrm{~W} / \mathrm{cm}^{2}$, very close to the observed value.

The previous measurement of the amplitude of density fluctuations associated with the primary SRS-EPWs (Sec. III) have to be corrected to take into account that EPWs are localized in speckles that represent a smaller scattering volume than the geometrical volume used before. To do so we need to calculate the number of active SRS speckles that contribute to the probed volume. The above formula $M_{3}(u)$ describes the probability distribution for the speckles centers in a three-dimensional space while in this experiment, the width of the CTS probed volume $(\delta z=75 \mu \mathrm{m})$ was narrower than the mean speckle length $\left(1_{s}=250 \mu \mathrm{m}\right)$. For the case $\delta z<1_{s}$, one has to use the two-dimensional probability distribution function $M_{2}(u) \sim\left(S_{\text {scatt }} / S_{\text {sp }}\right) u^{1 / 2} e^{-u^{2}}$, 25 where, instead of the ratio of volumes in $M_{3}(u)$, we have the ratio of surfaces in the plane across the direction of propagation of the interaction laser beam, $S_{\mathrm{scar}} / S_{\mathrm{sp}} \approx 330$. Then we can calculate the number of speckles that contribute to the Thomson scattered light, $M_{2}(5) \sim 5$, speckles. Therefore the scattered signal is collected from approximately five speckles and their volume is approximately 70 times less than the total probed volume. Because of that the amplitude of the density fluctuations, $(\delta n / n)_{\text {epwl }} \sim\left(I_{\text {scart }} / I_{\text {probe }}\right)^{1 / 2}\left(V_{\text {scart }}\right)^{-1 / 2}$, has to be corrected by a factor of $\sim 9$, which is the square root of the ratio of the probed and scattered volumes.

The real amplitude of SRS-EPWs, averaged in time, is then $\geqslant 9 \times 10^{-3}$, which is above the calculated LDI threshold. Probably, the local EPW amplitude could be even larger than this estimate. This last argument comes from inhomogeneity of the laser light intensity across the speckle and from the observed shortening of the TS signal associated with secondary EPWs compared to the one associated with primary EPWs, which shows that the laser intensity is above the threshold only for part of the Gaussian laser pulse. A marginal self-focusing in speckles can also contribute to additional enhancement of EPW amplitudes.

The occurrence of LDI at low plasma densities $\left(n_{e} / n_{c}\right.$ $\leqslant 5 \%$ ) is due to the electron temperature decreasing along with the plasma density, which is in agreement with the adiabatic cooling of the plasma due to expansion at this time of the laser pulse. From Fig. 8, the laser intensity is above the threshold for a temperature of $\sim 0.2-0.3 \mathrm{keV}$. The vanishing of the scattered signal associated with the secondary EPWs at $n_{e} / n_{c}=3 \%$ during the second half of the scattered signal associated with the primary EPWs shows that the LDI threshold increases toward lower densities, and it is marginally reached at this density. Hence the LDI can be suppressed by minor modifications of the plasma conditions.

It is possible to connect the primary EPW's amplitude at the threshold with the SRS level. According to the linear SRS theory," and assuming strong EPW damping, we have

$$
\left(\frac{\delta n}{n}\right)_{\text {epw } 1} \approx \frac{1}{4} \frac{\omega_{\text {epw }}}{\nu_{\text {epw }}}\left(k_{\text {epw }} \lambda_{\mathrm{D}}\right)^{2}\left(\frac{v_{0}}{v_{T e}}\right)^{2} \sqrt{R_{\mathrm{SRS}}},
$$

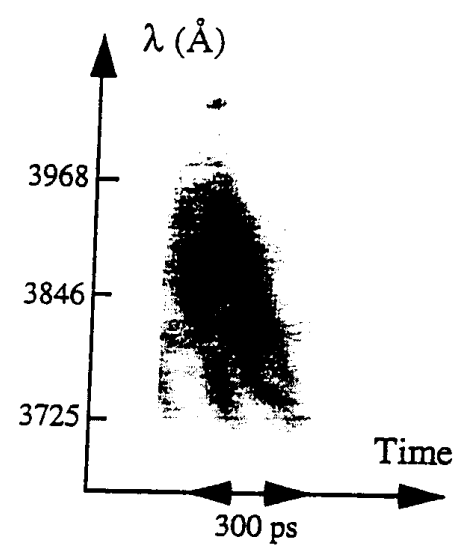

FIG. 9. The time-resolved spectrum of Thomson scattered light from electron plasma waves driven by stimulated Raman scattering showing temporal and spectral strucrures. The laser intensity is $7 \times 10^{13} \mathrm{~W} / \mathrm{cm}^{2}$.

where $v_{0} / v_{T e}$ is the ratio of quiver electron velocity to thermal velocity. We assume that nonlinear effects are negligible, as they will come mainly from the secondary EPWs and their amplitude is very small. For our parameters and

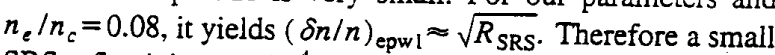
SRS reflectivity, $\sim 10^{-4}$, is enough to excite LDI. This estimate also explains why the observed thresholds for SRS and LDI are close. The occurrence of LDI modifies the SRS growth. and eventually leads to SRS saturation. It has also been observed in simulations that LDI demonstrates nonstationary pulsating behavior near its threshold. ${ }^{11-14}$ This can turn off SRS by increasing the damping of the EPWs, leading to a dynamical evolution of the instability on a short time scale ( of the order of a picosecond). Temporal bursts observed in the Thomson scattered spectra of EPWs associated with SRS, as shown in Fig. 9, could be a signature of this effect. Although the temporal resolution of the streak camera cannot resolve one picosecond, the observed temporal modulations of the EPW could be an indication of the interplay between SRS and LDI. This type of structure has already been observed in many past experiments under different interaction conditions.

The last point to be discussed is the ratio of amplitudes of secondary and primary EPWs. From theories ${ }^{10-13}$ of SRS saturation due to LDI cascading and/or strong Langmuir turbulence in homogeneous plasmas and for homogeneous laser intensity, it is expected that LDI should produce secondary EPWs of comparable amplitude as the primary ones, and eventually a cascade of Langmuir waves will take place as long as the amplitude of the primary EPW is at least twice above the LDI threshold. Even if the cascade would occur in the experiment, it would not be possible to see it with the TS diagnostics, because the scattered light from the subsequent cascades would be superimposed to the already present signals from primary and secondary EPWs. The TS diagnostics does not have enough spectral resolution to separate these contributions. As the previous estimates have demonstrated that, in our conditions, SRS originates from the highestintensity speckles, a possible explanation of the observed 
small ratio of EPW's amplitudes could be that the number of speckles that contribute to the upshifted signal in the probed volume is much smaller than the number of speckles that contribute to the downshifted TS signal because primary EPW amplitude could be below the LDI threshold for part of the SRS-active speckles. The measured ratio would then be an indication of the relative number of active speckles for these two instabilities.

To illustrate the results of the statistical theory on the SRS saturation and the secondary LDI, we have developed a simple semiqualitative model. We assume that the EPW intensity increases exponentially with the speckle intensity below the LDI threshold, according to standard theory of convective SRS. Above the LDI threshold, the EPW intensity growth slows down. We approximated EPW1 and EPW2 intensities above the LDI threshold with linear functions of the pump intensity, assuming that amplitudes of both EPWs are comparable for laser intensities twice above the LDI threshold. This is in agreement with the theory and numerical simulations of SRS saturation due to LDI. ${ }^{11-14}$ Then the expression for EPW1 intensity is

$$
(\delta n / n)_{\mathrm{epw} 1}^{2} \sim \exp \left(2 G_{R} u\right) \text { if }(\delta n / n)_{\mathrm{epw} 1}^{2}<(\delta n / n)_{\mathrm{LDIth}}^{2}
$$

and

$$
\begin{aligned}
(\delta n / n)_{\text {epw1 }}^{2}= & (\delta n / n)_{\text {LDIth }}^{2}\left[1+\alpha G_{R}\left(u-u_{\mathrm{th}}\right)\right], \\
& \text { for }(\delta n / n)_{\mathrm{epw} 1}^{2}>(\delta n / n)_{\text {LDIth. }}^{2} .
\end{aligned}
$$

Here $G_{R}$ is the SRS gain calculated for the average laser intensity, $\alpha$ is a numerical coefficient of the order of $0.3, u_{\text {th }}$ is the speckle at the LDI threshold. Correspondingly, the secondary EPW is absent for $u<u_{\mathrm{th}}$ and increases linearly with $u$ above it:

$$
(\delta n / n)_{\mathrm{epw} 2}^{2}=(\delta n / n)_{\mathrm{LDIth}}^{2} \alpha G_{R}\left(u-u_{\mathrm{th}}\right), \text { for } u>u_{\mathrm{th}} .
$$

The EPW intensities were then averaged with the probability distribution function $M_{2}(u)$ assuming that there is no speckle above some maximum intensity $u_{\max }-7$, and that the LDI threshold is 1.4 times above the SRS threshold. The resuit of this model, which is shown in Fig. 10(a), illustrates the dependence of the primary EPW intensity on the SRS average gain. It shows a faster growth of the EPWI intensity below the LDI threshold than above it, with a change of the slope by a factor of 2 . The ratio of secondary to primary EPW amplitudes is shown in Fig. 10(b). This ratio is small, like experimental values, for average intensities very close to the LDI threshold, and increases as a function of the laser intensity, as it had been observed in the experiment, but with a larger slope. More measurements, with higher laser intensities, would be of great interest to push further this modeling.

\section{CONCLUSIONS}

In summary, using Thomson scattering of a shortwavelength probe beam, two EPWs with the same frequency and propagating in opposite directions have been simultaneously observed. The primary EPWs, copropagating with the laser beam, are associated with the SRS instability, while the secondary EPWs have been identified as a LDI product.
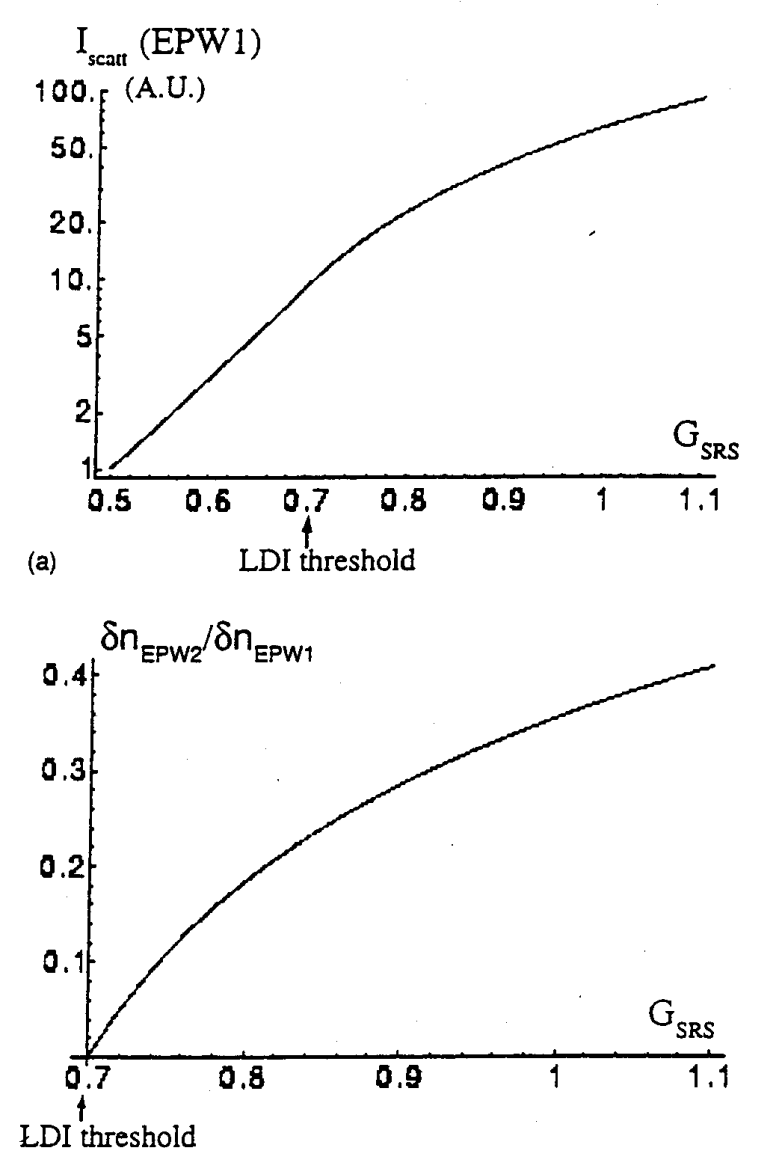

(b)

FIG. 10. (a) Dependence of the averaged intensity of the primary EPW on the average SRS gain calculated from the simple model described in the text for $u_{\max }=7, \alpha=0.3$. It is assumed that the LDI threshold is achieved at the maximum intensity speckle for $G_{R}=0.7$. The gain $G_{R}=0.5$ corresponds to the SRS threshold. (b) The racio of secondary to primary EPW average amplitudes as a function of average SRS gain. Parameters are the same as in (a).

Other possible mechanisms of production of the secondary EPWs have been analyzed and ruled out. This experiment demonstrates that even small density fluctuations associated with Langmuir waves can produce the Langmuir decay instability and that its threshold is close to the threshold of the Raman instability. This shows that decay of the Langmuir waves has to be included in the modeling of SRS, even in the case of low reflectivities. More measurements on the change of the SRS gain as a function of the pump intensity are needed to improve modeling of the saturation of SRS. Theoretical analysis including randomly distributed speckles has improved a lot the modeling of an experiment. Nevertheless, from our results on the SRS threshold and on the SRS gain, it looks that self-focusing of the laser light in speckles has to be included in the modeling, which will be the next step of this program. Self-focusing could produce a density depletion inside the speckles that could be deep enough to trap and back reflect the primary SRS EPWs. The maximum measured ratio of the amplitudes of the density fluctuations associated with secondary and primary EPWs is smaller than 
what the cascade theory of SRS saturation predicts. This theory applies to homogeneous plasma and intensity distribution and could be reconsidered for the case of more realistic plasmas.

\section{ACKNOWLEDGMENTS}

The authors gratefully acknowledge very useful discussions with $\mathrm{K}$. Baker and $\mathrm{K}$. Estabrook, and the support of A. Michard and the technical groups of LULI during the experiment. This work was partially supported under the auspices of the U.S. Department of Energy by the Lawrence Livermore National Laboratory under Contract No. W-7405ENG-48.

Part of this support was provided through the LLNLLDRD program under the Institute for Laser Science and Applications.

${ }^{1}$ M. V. Goldman, Rev. Mod. Phys. 56, 709 (1984).

${ }^{2}$ W. L. Lindl, Phys. Plasmas 2, 3933 (1995).

${ }^{3}$ W. L. Kruer, The Physics, of Laser Plasma Interactions (Addison-Wesley, Redwood City, CA, 1988).

${ }^{4}$ E. J. Valeo and W. L. Kruer, Phys. Fluids 16, 675 (1973).

${ }^{5} \mathrm{~V}$. Yu. Bychenkov, V. P. Silin, and V. T. Tikhonchuk, JETP Lett. 26, 197 (1977): V. P. Silin and V. T. Tikhonchuk, Phys. Rep. 135, 1 (1986).

${ }^{6}$ D. F. DuBois and M. V. Goldman, Phys. Rev. Lett. 14, 544 (1965); D. F. DuBois and M. V. Goidman, Phys. Rev. 164, 207 (1967).

${ }^{7}$ H. A. Baldis, E. M. Campbell, and W. L. Kruer, "Laser-plasma interactions." in Phvsics of Laser Plasmas, Handbook of Plasma Physics (NorthHolland, Amsterdam, 1991), pp. 361-434; R. P. Drake, R. E. Tumer, B. F. Lasinski, E. A. Williams, D. W. Phillion, K. Estabrook, W. L. Kruer, E. M. Campbell, T. W. Johnston, K. R. Manes, and J. S. Hildum, Phys. Fiuids 31, 3130 (1988).

${ }^{8}$ D. E. Evans and J. Katzenstein. Rep. Prog. Phys. 32, 207 (1969); J. Sheffield, Plasma Scattering of Electromagnetic Radiation (Academic Press, New York, 1975); R. E. Slusher and C. M. Surko, Phys. Fluids 23, 472 (1980); E. E. Salpeter, Phys. Rev. 120, 1528 (1960).
${ }^{9}$ R. P. Drake and S. H. Batha, Phys. Fluids B 3, 2936 (1991).

${ }^{10}$ D. Villeneuve, K. L. Baker. R. P. Drake, B. Sleaford. B. L. La Fontaine. K. Estabrook. and M. K. Prasad, Phys. Rev. Lett. 71. 3, 368 (1993).

${ }^{11}$ J. A. Heikkinen and S. J. Karttunen, Phys. Fluids 29. 1291 (1986).

${ }^{12}$ G. Bonnaud, D. Pesme, and R. Pellat. Phys. Fluids B 2. 1618 (1990).

${ }^{13}$ H. A. Rose. D. F. DuBois, and B. Bezzerides, Phys. Rev. Lett. 58. 2547 (1987); B. Bezzerides, D. F. DuBois. and H. A. Rose, ibid. 70, 2569 (1993).

${ }^{14}$ T. Kolber, W. Rozmus, and V. T. Tikhonchuk, Phys. Fluids B 5, 138 (1993).

${ }^{15}$ W. Seka. R. S. Craxton, C. Labaune, H. A. Baidis, N. Renard, E. Schifano, and A. Michard, Bull. Am. Phys. Soc. 40, 11, 1777 (1995).

${ }^{16} \mathrm{H}$. A. Baldis and C. Labaune, Rev. Sci. Instrum. 67, 45I (1996).

${ }^{17}$ C. Labaune, H. A. Baldis, N. Renard, E. Schifano, S. D. Baton, A. Michard, W. Seka, R. Bahr, B. S. Bauer, K. Baker, and K. Estabrook, Phys. Rev. Lett 75, 248 (1995).

${ }^{18}$ N. Renard, C. Labaune, H. A. Baldis, B. S. Bauer, B. Quesnel, E. Schifano. A. Michard, W. Seka, and K. G. Estabrook, Phys. Rev. Lett. 77, 3807 (1996).

${ }^{19}$ K. L. Baker, R. P. Drake, B. S. Bauer, K. G. Estabrook, A. M. Rubenchik, C. Labaune, H. A. Baldis. N. Renard, S. Baton, E. Schifano, A. Michard. W. Seka, and R. E. Bahr, Phys. Rev. Lert. 77, 67 (1996).

${ }^{20} \mathrm{~W}$. Rozmus, A. A. Offenberger, and R. Fedosejevs, Phys. Fluids 26, 1071 (1983); K. Estabrook, W. L. Kruer, and M. G. Haines. Phys. Fluids B 1 , $1282(1989)$

${ }^{21}$ P. K. Kaw, A. T. Lin, and J. M. Dawson, Phys. Fluids 16, 1967 (1973); H. C. Barr and F. F. Chen, ibid. 30, 1180 (1987).

${ }^{22}$ C. Labaune, H. A. Baldis, N. Renard, E. Schifano, and A. Michard. Phys. Plasmas 4, 423 (1997).

${ }^{23}$ A. Simon and R. W. Shor, Phys. Fluids B 1.1073 (1989).

${ }^{24}$ E. A. Williams, R. L. Berger, R. P. Drake, A. M. Rubenchik, B. S. Bauer. D. D. Meyerhofer, A. C. Gaeris, and T. W. Johnston, Phys. Plasmas 2, 129 (1995).

${ }^{25}$ H. A. Rose and D. F. DuBois, Phys. Fluids B 5, 590 (1993).

${ }^{26}$ P. N. Guzdar, C. S. Liu, and R. H. Lehmberg, Phys. Fluids B 5, 910 (1993).

${ }^{27}$ R. W. Short, W. Seka, and R. Bahr, Phys. Fluids 30, 3245 (1987).

${ }^{28}$ V. T. Tikhonchuk, C. Labaune, and H. Baldis, Phys. Plasmas 3, 3777 (1996).

${ }^{29} \mathrm{H}$. Rose, Phys. Plasmas 2, 2216 (1995). 


\title{
Suppression of stimulated Brillouin scattering by seeded ion wave mode coupling
}

\author{
B. I. Cohen, B. F. Lasinski, A. B. Langdon, E. A. Williams, and H. A. Baldis \\ Lawrence Livermore National Laboratory, University of California, Livermore, California 94550 \\ C. Labaune \\ Laboratoire pour L'Utilisation des Lasers Intenses, Centre National de la Recherche Scientifique, Ecole \\ Polytechnique, 91128 Palaiseau Cedex, France
}

(Received 10 February 1998; accepted 22 May 1998)

Particle simulations and the analytical solution of coupled mode equations are used to demonstrate that the mode coupling of a primary ion acoustic wave produced by stimulated Brillouin backscatter with a secondary finite-amplitude ion wave propagating at a finite relative angle can reduce the amplitude of the primary ion wave and the backscatter. The simulations and analysis give results that are in qualitative agreement with experimental observations [Baldis et al., Phys. Rev. Lett. 77, 2957 (1996)] of the partial suppression of stimulated Brillouin backscattering (SBBS) when there are simultaneous, overlapping SBBS processes at a finite relative angle, which may influence SBBS reflectivities in other current and future laser-fusion experiments with multiple overlapping beams. (c) 1998 American Institute of Physics. [S1070-664X(98)00309-7]

\section{INTRODUCTION}

In laser-plasma experiments at the Laboratoire pour L'Utilisation des Lasers Intenses (LULI), ${ }^{1}$ it was observed that the primary ion acoustic wave (IAW) produced by stimulated Brillouin backscattering (SBBS) of one pump laser beam could be substantially reduced in amplitude in the presence of a secondary SBBS process involving a second (seed) laser beam propagating at a $22.5^{\circ}$ relative angle. This observation and its elucidation are of significant interest; the study and control of SBBS have been the object of continuous theoretical and experimental research for many years because of the importance of SBBS in laser fusion. ${ }^{2-13}$ The symmetric compression of fusion targets in either direct drive or indirect drive can be affected by SBBS and other parametric instabilities unless these instabilities are controlled. Furthermore, in order to optimize the fusion performance of experiments proposed in the National Ignition $\mathrm{Fa}$ cility (NIF), ${ }^{14}$ which will have multiple crossing laser beams and require careful control over the timing and relative amplitudes of the crossing beams, the interaction of simultaneous SBBS events in crossing laser beams should be understood. Finally, the nonlinear interaction of driven waves in a plasma is of fundamental interest.

In this research, we make use of the BZOHAR twodimensional, hybrid (particle ions and Boltzmann fluid electrons) simulation code introduced in earlier work. ${ }^{15}$ As described in Ref. 15, Poisson's equation with a nonlinear Boltzmann electron response is solved in two spatial dimensions for the self-consistent scalar electric potential with the ion density collected from particle ion positions; and the Maxwell curl equations are reduced via a temporal envelope approximation to a Schrödinger-like equation for the highfrequency transverse wave amplitude. The electron ponderomotive potential due to the transverse waves is included in the electron Boltzmann response. Here, using model simula- tions and a mode coupling analysis, we propose a mechanism that can explain some of the LULI observations. The simulations and the analysis indicate that mode coupling of the ion waves in the primary and secondary SBBS processes leads to enhanced damping of the ion waves which reduces their amplitudes and the SBBS reflectivities as observed in the LULI experiments. In some sense this work is a logical extension of the earlier work of Maximov et al. which addressed the effects of long wavelength velocity and density perturbations in detuning SBS. ${ }^{16}$

\section{SIMULATIONS AND ANALYSIS}

The interaction between seeded and primary IAWs is most easily studied in two-dimensional, doubly periodic, electrostatic simulations of ponderomotively driven ion waves (Figs. 1 and 2). In Fig. 1 are results from three simulations in which a primary IAW was resonantly excited by an imposed electron ponderomotive potential with amplitude $e \phi_{0} / T_{e}=0.05, k_{s} \lambda_{e}=0.1$, and $T_{e} / T_{i}=10.27$ in a $\mathrm{CH}$ plasma (plasma parameters motivated by the LULI experiments), where $\lambda_{\mathrm{De}}$ is the electron Debye length, and $T_{e}$ and $T_{i}$ are the electron and ion temperatures. In the first simulation there was no seeded secondary IAW, and the primary IAW relaxed via a decay instability ${ }^{15}$ after being driven to a large amplitude. In the second and third simulations, a secondary IAW was excited at nearly the same frequency but at a relative angle of $18^{\circ}$ and with driver amplitudes $e \phi_{\text {seed }} / T_{e}=0.0025$ and 0.02 , respectively. The primary and secondary wave driving amplitudes were turned on smoothly over two acoustic periods and left on. The collapse of the primary IAW in the first simulation when no seed is present is caused by the parametric decay into two ion waves at the half-harmonic of the primary IAW's $k_{x}$ wave number and with finite, but generally smaller, $k_{y}$. The two-ion-wave parametric decay has been described in Ref. 15 and elsewhere. ${ }^{17}$ The primary ion 
(a)

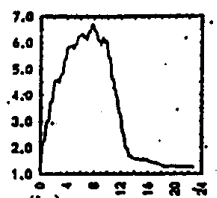

(b)

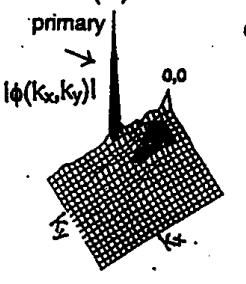

no seed

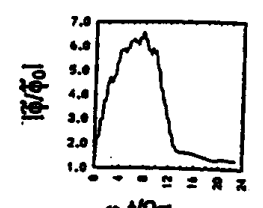
$\omega_{0} 4 / 2 \pi$

$e \phi_{0} T_{0}=0.05$

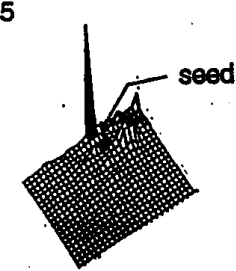

$0 \phi_{0000} / T_{0}=0.0025$
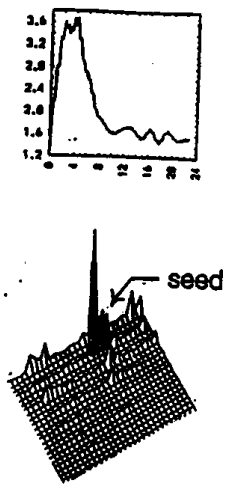

$\theta \phi_{\text {seed }} / T_{\theta}=0.02$
FIG. 1. (a) The ratio of the Fourier amplitudes at the driving frequency and wave number of the plasma electric potential and the electron ponderomotive potential $\left|\bar{\phi} / \bar{\phi}_{0}\right|$ as a function of time for $e \phi_{0} / T_{e}=0.05$ and $e \phi_{\text {seed }} / T_{e}=\{0,0.0025,0.02\}$, and (b) the corresponding $\left|\phi\left(k_{x}, k_{y}\right)\right|$ vs $k_{x}$ and $k_{y}$ at $\omega_{s} t / 2 \pi=12$ with a $128 \Delta x$ $\times 128 \Delta y$ domain, 64 particles per species per cell, $k_{s} \lambda_{D_{e}}=0.1$ for the driven wave, acoustic driving frequency $\omega_{s}=k_{s} c_{s} /\left(1+k_{s}^{2} \lambda_{\mathrm{De}}^{2}\right)^{1 / 2}$, where $c_{s}$ is the sound speed, and seed wave at $18^{\circ}$. wave response to its driving potential as a function of time was only minimally reduced by the secondary seeding with $e \phi_{\text {seed }} / T_{e}=0.0025$. However, the primary IAW was reduced substantially for $e \phi_{\text {seed }} / T_{e}=0.02$ so that the primary LAW
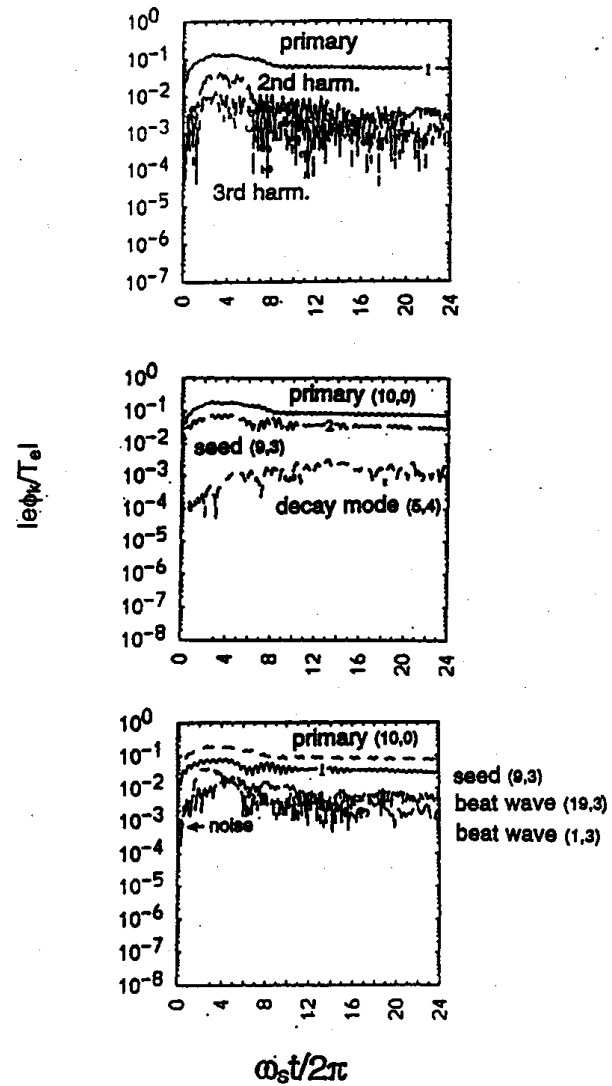

FIG. 2. Time histories of the amplitudes $\left|e \phi_{k} / T_{e}\right|$ of the primary IAW, its second and third harmonics, the seed wave, a decay mode of the primary, and the beats of the seed and primary IAW for the strongly seeded case in Fig. 1. The number pairs are labels indicating the $x$ and $y$ mode numbers of the waves in the simulation. did not exceed the threshold for the decay instability. ${ }^{15}$ The seed IAW amplitudes were never big enough to exceed this threshold. In Fig. 2 we present the time histories of the amplitudes of the primary, seed, and beat waves of the primary and seed in the strongly seeded case. The beats of the primary and strong seed grew at the expense of the primary and the seed, and all of the modes ultimately were damped by heating the ions. In the strongly seeded case; the decay modes of the primary IAW, which were observed to grow to amplitudes $\left|e \phi_{k} / T_{e}\right| \sim 0.05$ for the weakly driven seed, achieved amplitudes $\left|e \phi_{k} / T_{e}\right| \leqslant 2 \times 10^{-3}$, which was typical of the simulation noise level in these driven plasmas. The amplitudes of the second and third harmonics of the primary wave tracked the growth and relaxation of the primary in this strongly seeded case. There was significant ion trapping and acceleration of a fast ion tail due to the action of the primary wave. ${ }^{5,7,15}$

The peak primary ion wave amplitudes for the simulations shown in Fig. 1 were $\left|e \phi_{k} / T_{e}\right| \sim 0.15-0.3$ and then relaxed to $\left|e \phi_{k} / T_{e}\right| \sim 0.05$ on the time scale of 5-10 acoustic periods due to the action of ion wave parametric decay or mode coupling and damping on the ion velocity distribution function. This is a relatively fast time scale and is shorter than the 50 ps temporal resolution of the Thomson scattering used to diagnose the ion wave amplitudes in the LULI experiments. The LULI measurements suggested ion wave amplitudes no larger than $\left|\delta n_{e} / n_{0}\right| \sim\left|e \phi / T_{e}\right| \sim 0.1$. The strength of the mode coupling depends on the product of the primary and secondary IAW amplitudes. With $e \phi_{0} / T_{e}$ $=0.01$, which led with no secondary seeding to a peak response $\left|\delta n_{e} / n_{0}\right|=0.1$ like the LULI observations, and seeding with $e \phi_{\text {seed }} / T_{e}=0.003$, we observed in the simulation a peak primary wave amplitude $\left|\delta n_{e} / n_{0}\right|=0.08$, i.e., a $20 \%$ reduction due to mode coupling with this small seed IAW.

The electrostatic simulations illustrate that mode coupling with the seed IAW provides an additional loss channel for the primary IAW (and also for the seed). The following mode coupling analysis illustrates how the coupling of the ion waves leads to additional dissipation for the primary 
IAW that reduces its amplification by SBBS and reduces the SBBS reflectivity. Consider the following simplified set of coupled mode equations modeling resonant SBBS and heavily damped ion waves in a uniform plasma in steady state:

$$
-v_{g} \frac{\partial a_{1}}{\partial x}=c_{1} a_{0}^{*} a_{s 1} \text { backscattered transverse wave, }
$$

$$
\frac{d a_{s 1}}{d t}=c_{2} a_{0} a_{1}-\gamma_{s 1} a_{s 1}-c_{3} a_{s 2} a_{s 3}=0 \text { primary IAW, }
$$

$$
\frac{d a_{s 3}}{d t}=c_{4} a_{s 1} a_{s 2}^{*}-\left(\gamma_{s 3}+i \Delta_{s 3}\right) a_{s 3}=0 \text { beat-wave IAW, }
$$

where $c_{1}, c_{2}, c_{3}$, and $c_{4}$ are coupling coefficients that can be identified in the analyses of $\mathrm{SBBS}^{2-4,6,7}$ and the two-ionwave parametric decay; ${ }^{15} v_{g}$ is the group velocity of the backscattered electromagnetic wave; $d a / d t$ is a convective time derivative on a wave amplitude $a ; \gamma_{s 1}$ and $\gamma_{s 2}$ are linear dissipation rates for the ion waves; $a_{0}$ and $a_{1}$ are the amplitudes of the pump and backscattered electromagnetic waves; $a_{s 1}, a_{s 2}$, and $a_{s 3}$ are primary, seed, and beat-wave ion wave amplitudes; and $\Delta_{s 3}$ is a frequency mismatch. The mode coupling equations are readily solved in steady state with the seed amplitude $a_{s 2}$ a fixed parameter; from Eqs. (1b) and (1c)

$$
a_{s 3}=\frac{c_{4} a_{s 1} a_{s 2}^{*}}{\gamma_{s 3}+i \Delta_{s 3}}, \quad a_{s 1}=\frac{c_{2} a_{0} a_{1}}{\gamma_{s 1}^{n l}},
$$

where $\operatorname{Re} \gamma_{s 1}^{n l}$ is the effective damping of the primary IAW,

$$
\gamma_{s 1}^{n l}=\gamma_{s 1}+\frac{c_{3} c_{4}\left|a_{s 2}\right|^{2}}{\gamma_{s 3}+i \Delta_{s 3}}
$$

The second term in Eq. (3) represents the enhancement of the damping of the primary IAW arising from the mode coupling induced by the seed. A spatial growth rate for SBBS is obtained from Eqs. (1a) and (2):

$$
\kappa=-\frac{\partial}{\partial x} \ln \left(a_{1}\right)=\frac{c_{1} c_{2}\left|a_{0}\right|^{2}}{v_{g} \gamma_{s 1}^{n l}}=\frac{\gamma_{\mathrm{SBS}}^{2}}{v_{g} \gamma_{s 1}^{n l}},
$$

where $\gamma_{\text {SBS }}$ is the homogeneous-medium temporal growth rate for weakly coupled Brillouin backscatter. ${ }^{2-4,6,7}$ The exponential amplification of both the backscattered electromagnetic wave amplitude and the primary IAW amplitude in the backward direction over a length $L$ is given by $\exp (\kappa L)$. The gain exponent $G \equiv \kappa L$ has been altered by the mode coupling induced by the seed IAW from the gain exponent in the absence of the seed, $G_{0} \equiv \gamma_{\mathrm{SBS}}^{2} L / v_{g} \gamma_{s 1}$ :

$$
G=\frac{\gamma_{s 1}}{\gamma_{s 1}^{n l}} G_{0}
$$

The mode coupling has led to enhanced damping of the primary ion wave if $\left|\gamma_{s 1}^{n l} / \gamma_{s 1}\right|>1$; and the gain exponent is reduced correspondingly. If there is a $20 \%$ enhancement of the effective dissipation, as suggested by the $20 \%$ reduction of the primary IAW realized in our ponderomotively driven electrostatic simulations using parameters appropriate to the LULI experiment, then for $G_{0} \sim 5$, which is a typical value for the LULI experiment with laser intensity $I_{0}$ $=10^{14} \mathrm{~W} / \mathrm{cm}^{2}$ at $1 \mu \mathrm{m},{ }^{18}$ the square of the relative density perturbation in the primary IAW is expected to be reduced by $[\exp (-5 \times 0.2)]^{2}=0.14$. This estimate is in rough agreement with the LULI observations. ${ }^{1}$

If the seed LAW leads to enhanced damping of the primary IAW via mode coupling, we expect a similar effect on the seed due to the action of the primary IAW. This is observed in the time histories in Fig. 2 where, after the primary and seed LAW amplitudes are driven to finite amplitudes, they both decay accompanying and following the growth of the beat waves of the seed and primary IAWs. This can be understood by augmenting the coupled mode equations, Eqs. (1a), (1b), and (1c), to include a dynamical equation for the seed IAW of the same form as Eq. (1b). The seed IAW also acquires an enhanced damping rate arising from the mode coupling induced by the primary and the seed of the same form as in Eq. (3).

The seed IAW in the LULI experiments is excited by the interaction of a seed laser beam propagating at a finite -relative angle with respect to the primary laser beam. In our simulations we have considered only the effects of a single specific seed IAW with the backscatter IAW of the primary laser beam. The work of Dubois, Bezzerides, and Rose ${ }^{19}$ analyzes the collective parametric instabilities of many overlapping and converging laser beams. Their formulation is a useful reference for understanding the following qualitative arguments. The Thomson scattering diagnostics in the LULI experiments reveal that for a weak seed laser strength, the backscatter IAW is the dominant IAW. ${ }^{1}$ Therefore, we focus on the mode coupling of the backscatter IAWs of both the primary and the secondary laser beams in this work. However, in the LULI experiments there was also the mutually resonant IAW propagating at an angle that bisected the angle between the propagation directions of the two laser beams. This mutually resonant IAW was jointly driven by the SBS ponderomotive forces of the primary and seed laser beam, and it was not surprising that its amplitude was enhanced as the seed laser strength was increased. The mutually resonant IAW amplitude squared scaled approximately linearly with seed laser intensity suggesting that the seed and primary SBS ponderomotive forces added incoherently. ${ }^{19}$ Figures $2-4$ of Ref. 1 indicate that the primary backscatter IAW amplitude for zero seed laser intensity was larger over a bigger volume than was the amplitude of the mutually resonant IAW, which was enhanced over only a very narrow cone of angles, and that the suppression effect of the backscatter IAW due to the nonresonant seed was more dramatic than was the enhancement effect of the mutually resonant IAW. The overall reflectivities in the LULI experiment were relatively low, $\sim 5 \%$; and pump depletion was not believed to be significant, except perhaps over a short-lived transient time scale that the diagnostics could not resolve in the experiments. ${ }^{18}$

In the simulation studies reported here, only a single 
(a)
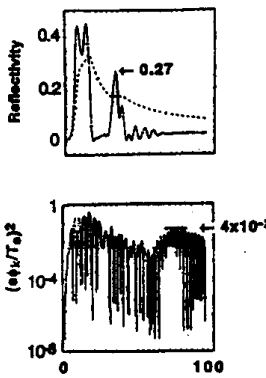

(b)
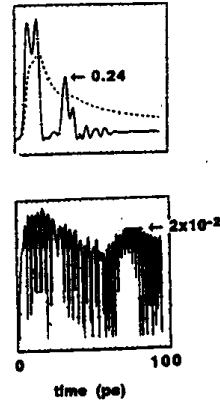

(c)
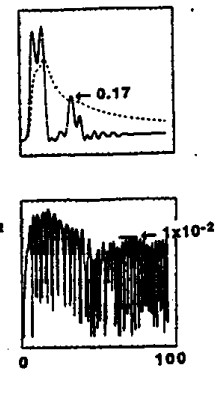

FIG. 3. Time histories of the instantaneous (solid curve) and cumulative average (dotted) reflectivities and primary IAW amplitudes $\left|e \phi_{k} / T_{e}\right|^{2}$ as functions of time for (a) $e \phi_{\text {seod }} / T_{s}=0.01$, (b) $e \phi_{\text {seed }} / T_{e}=0.07$, and (c) $e \phi_{\text {seod }} / T_{e}=0.14$ in a CH plasma with $m_{H} / m_{e}=1836, T_{e} / T_{i}=2.4, k_{j} \lambda_{D_{e}}$ $=0.2$, laser electron quiver velocities $v_{0}^{L} / v_{e}=0.577$ for the input value of the primary laser beam incident from the left side of the simulation, $v_{0}^{R} / v$ e $=0.0577$ for the input value of the backscatter wave at the right side of the simulation, $v_{e}=\left(T_{e} / m_{e}\right)^{1 / 2}$, and electron density equal to $10 \%$ critical density. The time histories of $\left|e \phi_{k} / T_{e}\right|^{2}$ were computed from the Fourier transform of the electric potential with respect to $y$ at a position $x=L_{x} / 4$.

seed IAW and a single primary IAW are considered rather than a spectrum of IAWs propagating at various angles. We present quantitative arguments for the efficacy of the seed IAW associated with the backscatter of the seed laser in suppressing the backscatter of the primary laser via mode coupling of the IAWs. We expect that the mutually resonant IAW also mode couples with the primary LAW and aids in the suppression effect, because the physics of the mode coupling with the primary IAW is qualitatively the same for both the mutually resonant and the nonresonant seed IAWs. Thus, our model calculations likely underestimate the suppression effects of mode coupling on the backscatter of the primary by omitting the mutually resonant IAW. The inclusion of the mutually resonant IAW in our simulations and quantitative analysis is beyond the scope of this paper and will be left to future research.

To corroborate the suppression of SBBS due to mode coupling induced by a seed IAW, we performed electromagnetic simulations of SBBS with a ponderomotively driven plane-wave seed IAW propagating at $30^{\circ}$ with respect to the primary IAW excited by the SBBS. In these simulations only exactly forward and backward scattered electromagnetic waves were allowed $\left(k_{y}=0\right)$ with radiative (outgoing-wave) and charge-conserving boundary conditions in $x$ and periodicity in $y$. The mesh used was $2600 \Delta x \times 512 \Delta y$, with 25 particles per ion species per cell, $\lambda_{D_{e}} \sim \Delta x, T_{e}=0.6 \mathrm{keV}$, $T_{e} / T_{i}=2.4$ in a $\mathrm{CH}$ plasma, $L_{x} \sim 42 \lambda_{0}, L_{y} \sim 8 \lambda_{0}$, where $\lambda_{0}$ is the laser wavelength, and $k_{s} \lambda_{D e}=0.2$. The results of three electromagnetic simulations are shown in Fig. 3 for $e \phi_{\text {seed }} / T_{e}=\{0.01,0.07,0.14\}$ and a plane-wave laser beam corresponding to $10^{14} \mathrm{~W} / \mathrm{cm}^{2}$ at $1 \mu \mathrm{m}$ wavelength. The time histories of the instantaneous and cumulative time-averaged reflectivities, and the amplitudes $\left(e \phi_{k} / T_{e}\right)^{2}$ of the primary IAW in each case demonstrated a significant reduction of the reflectivities for $t>20$ ps and of the primary IAW amplitudes as $e \phi_{\text {seed }} / T_{e}$ was increased. We performed an additional

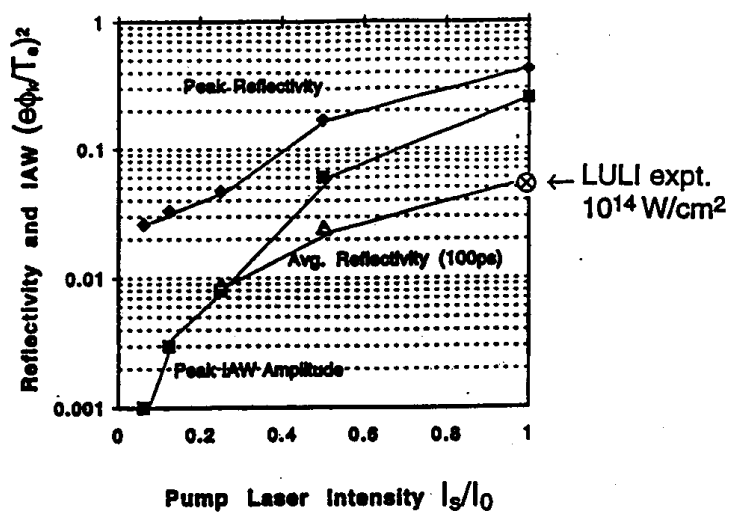

FIG. 4. Peak (solid $\diamond)$ and time-averaged $(0-100 \mathrm{ps}, \Delta)$ reflectivities and peak IAW amplitudes (a) $\left|e \phi_{k} / T_{e}\right|^{2}$ as functions of relative seed laser intensity $I_{s} / I_{0}$ for $I_{0}=10^{14} \mathrm{~W} / \mathrm{cm}^{2}$ from a series of two-dimensional SBBS backscatter simulations varying $v_{0}^{L} / v_{e}$ incident from the left and using $v_{0}^{R} / v_{e}=0.04$ for the backscatter incident from the right, and other parameters as in Fig. 3 with no IAW seeding.

simulation with no seed, $e \phi_{\text {seed }} / T_{e}=0$, and obtained the same results for the time histories of the backscatter reflectivities and the primary IAW amplitudes shown in Fig. 3 for $e \phi_{\text {seed }} / T_{e}=0.01$. These results illustrate that the suppression effects of the seed IAW required both finite amplitude and finite time in which to occur. The maximum observed reflectivities and IAW amplitudes achieved for $t<20$ ps were unaffected by the IAW mode coupling whose effects set in at a later time after the primary and seed IAWs acquired finite amplitudes. This can be understood by retaining time derivatives in Eqs. (1b) and (1c) and considering the dynamics. The first peak in the reflectivities and the subsequent relaxation was affected by several nonlinearities observed in the simulation diagnostics: ion trapping, transient ion wave harmonic generation, parametric decay of the primary IAW, ${ }^{15}$ and partial pump depletion and associated nonlinear oscillations in the reflectivity.

The LULI data demonstrated that the reduction of the primary LAW amplitude was directly correlated with the intensity of the seed laser beam driving the secondary SBBS process. To make connection between the results of Fig. 3 and the LULI data, we undertook a series of SBBS simulations with no IAW seeding in which the laser intensity was varied (Fig. 4). This is not the same as a self-consistent electromagnetic simulation of the two interacting SBS events (which is beyond the scope of this simulation model). However, because the mode coupling produced by both the seed and primary LAWs requires a finite time to have a damping effect after the seed and primary IAWs reach finite amplitude, the relation between the peak SBBS IAW amplitude and the single pump laser intensity and the use of the results of Fig. 3 provide a first approximation to the seed laser intensity needed to suppress the backscatter of the primary SBBS. The results for the peak instantaneous and the cumulative time-averaged (over $100 \mathrm{ps)} \mathrm{reflectivities} \mathrm{and} \mathrm{the} \mathrm{peak}$ IAW amplitude $\left(e \phi_{k} / T_{e}\right)^{2}$ are plotted in Fig. 4 as functions of the average seed laser intensity $I_{s} / I_{0}$ with no primary laser beam present, where $I_{0}=10^{14} \mathrm{~W} / \mathrm{cm}^{2}$ and the same 
(a)

LULI Seeded SBS Experiments



(b)

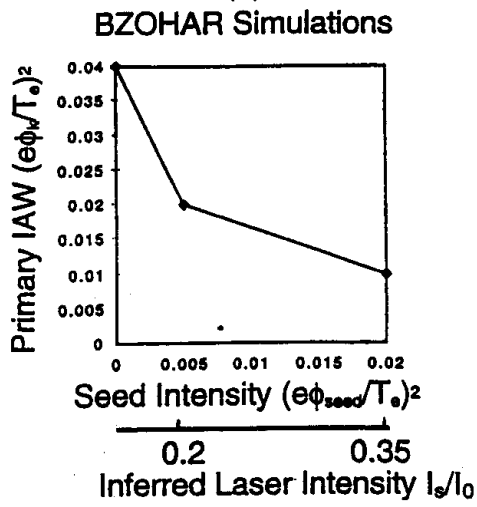

FIG. 5. (a) The square of the primary SBBS LAW amplitude in arbitrary units as a function of seed laser intensity from the LULI experiment (Ref. 1). (b) The square of the primary SBBS IAW amplitude $\left|e \phi_{k} / T_{e}\right|^{2}$ as a function of the seed LAW amplitude and the inferred laser intensity $I_{s} / I_{0}$ from the simulations in Figs. 3 and 4.
LULI plasma parameters were used as in Fig. 3. (The seed pump laser was incident from the left side of the simulation box. The input value of the backscattered electromagnetic wave at the right side boundary for the series of simulations shown in Fig. 4 was fixed at a relative intensity of $0.005 I_{0}$, and the reflectivities and concomitant peak primary IAW amplitudes were relatively insensitive to this input value.) The simulation reflectivity averaged over $100 \mathrm{ps}$ for $I_{s}=I_{0}$, the reference LULI intensity, was $5 \%$; and there was a sharp decrease in reflectivity with decreasing laser intensity, both of which results are in reasonable agreement with the LULI observations. ${ }^{18}$ For $I_{s} / I_{0}<0.5$ there was no ion wave decay instability observed in these simulations, because the peak amplitudes of the primary SBBS LAW were below threshold for the decay instability $\left(\left|e \phi_{k} / T_{e}\right|<0.2\right.$ in this case) ${ }^{15}$ In comparing particle simulations of SBBS to experiments, it is important to take into account the temporal resolution of the experimental measurements (typically $\geqslant 50 \mathrm{ps}$ ). The peak reflectivities and other transient features occurring on time scales less than $\sim 20 \mathrm{ps}$ in the simulations cannot be resolved in the laboratory experiments.

The results in Fig. 4 allow us to infer the corresponding seed laser intensity required to produce the seed IAW amplitude used to suppress the primary SBBS process. In Fig. 5 we compare the LULI data on backscatter suppression to our BZOHAR simulation results. The primary IAW amplitude $\left(e \phi_{k} / T_{e}\right)^{2}$ was reduced to $1 / 4$ its value at the inferred intensity $I_{s} / I_{0}=0.35$ compared to its amplitude with no seeding in the simulation, while the primary IAW was reduced to $1 / 6$ its unseeded value at an intensity $I_{s} / I_{0}=0.3$ in the LULI experiments. We expect that by including in our model the other ion waves (including the mutually resonant IAW) produced by the two interacting SBS events, there would be additional mode coupling and a further enhancement of the suppression of the backscatter of the primary pump laser. However, this is beyond the scope of our simulation model.

That the reflectivities and the SBBS suppression exhibited in the simulations are in rough agreement with the LULI observations is somewhat remarkable given the simplicity of the model. In addition to the omission of the angular spectrum of SBS, the actual laser intensity spatial structure was not incorporated into the simulations; and the simulation domains were smaller than a single speckle (hot spot) in the
LULI beams. However, the simulations did use appropriate spatially averaged laser intensities and plasma parameters. In Fig. 6 we show the results of a SBBS simulation that suggest that although the peak reflectivities and corresponding IAW amplitudes may depend on the domain (or speckle) length over which the instability amplifies initially, a nonlinear relaxation occurs to a much smaller length scale than the system length over time scales less than or equal to the temporal resolution of the measurements in the experiments. The picture that emerges from these simulations and multiplespeckle fluid simulations ${ }^{8}$ is that SBBS occurs as brief scintillations dominantly in laser hot spots, which give rise to bursts of reflectivity and large amplitude ion waves whose spatial domains of activity may rapidly shrink as the ion waves and the concomitant reflectivity relax with momentum and energy going from the laser to the ion waves and ultimately into the ion velocity distribution function. The experimental diagnostics integrate in space and time over multiple . SBBS scintillations. ${ }^{18}$

(a)
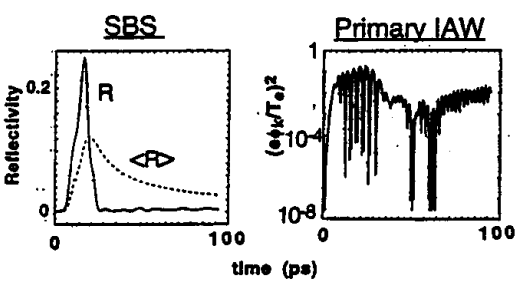

(b)
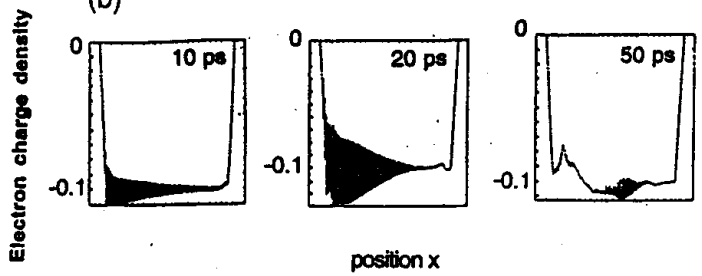

FIG. 6. (a) Instantaneous and cumulative time-averaged reflectivities and primary SBBS IAW amplitude $\left|e \phi_{k} / T_{e}\right|^{2}$ (computed from the Fourier transform of the electric potential with respect to $y$ at $x=L_{x} / 4$ ) as functions of time. (b) Snapshots of the $y$-averaged electron charge density as a function of position $x$ plotted over the entire $x$ domain at 10,20, and 50 ps from a simulation with backscatter input boundary condition $v_{0}^{R} / v_{e}=0.0115$, no IAW seeding, and otherwise the same parameters as in Fig. 3. 


\section{SUMMARY}

The simulations and analysis presented here indicate that a seeded ion wave can induce mode coupling of the seeded and SBBS ion waves (where the seed, SBBS, and beat ion waves are damped on the plasma) which substantially suppresses the SBBS reflectivity and its accompanying ion wave in qualitative agreement with the LULI experimental observations. Such a mechanism may mitigate SBBS activity in other experiments in which intense laser beams cross at finite angles.

\section{ACKNOWLEDGMENTS}

We are grateful to Richard Berger and other members of the scientific staff at the Lawrence Livermore National Laboratory for useful discussions, insight, and encouragement. We also thank the technical groups at LULI for their support. We acknowledge and thank the two referees for their comments regarding the potential importance of the mutually resonant ion waves in contributing to the reduction of the stimulated Brillouin backscatter of the primary laser beam and for their other valuable suggestions.

This work was performed for the U.S. Department of Energy under Contract No. W-7405-ENG-48 at the Lawrence Livermore National Laboratory.

${ }^{1}$ H. A. Baldis, C. Labaune, E. Schifano, N. Renard, and A. Michard, Phys. Rev. Lett. 77, 2957 (1996).

${ }^{2}$ V. P. Silin, Sov. Phys. JETP 21, 1127 (1965).

${ }^{3}$ J. F. Drake, P. K. Kaw, Y. C. Lee, G. Schmidt, C. S. Liu, and M. N. Rosenbluth, Phys. Fluids 17, 778 (1974).

${ }^{4}$ D. W. Forslund, J. M. Kindel, and E. L. Lindman, Phys. Fluids 18, 1002 (1975).

${ }^{5}$ D. W. Forslund, J. M. Kindel, and E. L. Lindman, Phys. Fluids 18, 1017 (1975).

${ }^{6}$ B. I. Cohen and C. E. Max, Phys. Fluids 22, 1115 (1979).
${ }^{7}$ W. L. Kruer, The Physics of Laser Plasma Interactions (Addison-Wesley, Reading, MA, 1988).

${ }^{8}$ R. L. Berger, B. F. Lasinski, A. B. Langdon, T. B. Kaiser, B. B. Afeyan, B. I. Cohen, C. H. Still, and E. A. Williams, Phys. Rev. Lett. 75, 1078 (1995).

${ }^{9}$ H. A. Baldis, D. M. Villeneuve, C. Labaune, D. Pesme, W. Rozmus, W. L. Kruer, and P. E. Young, Phys. Fluids B 3, 2341 (1991).

${ }^{10}$ H. A. Baldis, E. M. Campbell, and W. L. Kruer, in Handbook of Plasma Physics, Vol. 3, edited by A. Rubenchik (North-Holland, Amsterdam, 1990).

${ }^{11}$ H. A. Baldis, J. D. Moody, D. S. Montgomery, C. Labaune, S. H. Batha, K. G. Estabrook, R. L. Berger, E. A. Williams, B. F. Lasinski, W. L. Kruer, S. N. Dixit, B. Woods, M. A. Henesian, and E. J. Hsieh, Plasma Phys. Controlled Nucl. Fusion Res. 3, 221 (1992).

${ }^{12}$ L. V. Powers, R. L. Berger, R. L. Kaufman, B. J. MacGowan, P. A. Amendt, C. A. Back, T. P. Bernat, S. N. Dixit, D. I. Eimerl, K. G. Estabrook, J. A. Harte, D. H. Kalantar, D. E. Klem, B. F. Lasinski, D. S. Montgomery, J. D. Moody, D. H. Munro, T. D. Shepard, L. J. Suter, R. E. Turner, E. A. Williams, J. C. Fernandez, W. W. Hsing, B. H. Wilde, and B. H. Failor, Phys. Plasmas 2, 2473 (1995).

${ }^{13}$ B. J. MacGowan, B. B. Afeyan, C. A. Back, R. L. Berger, G. Bonnaud, M. Casanova, B. I. Cohen, D. E. Desenne, D. F. Dubois, A. G. Dulieu, K. G. Estabrook, J. C. Femandez, S. H. Glenzer, D. E. Hinkel, T. B. Kaiser, D. H. Kalantar, R. L. Kauffman, R. W. Kirkwood, W. L. Kruer, A. B. Langdon, B. F. Lasinski, D. S. Montgomery, J. D. Moody, D. H. Munro, L. V. Powers, H. A. Rose, C. Rousseaux, R. E. Turner, B. H. Wilde, S. C. Wilks, and E. A. Williams, Phys. Plasmas 3, 2029 (1996).

${ }^{14}$ National Technical Information Service Document Nos. DE95017671DE95017673 and DE95017676-DE95017700 (J. A. Paisner, E. M. Campbell, and W. J. Hogan, The National Ignition Facility Project, UCRL-JC117397 and UCRL-PROP-117093, May, 1994). Copies may be obtained from Nat. Tech. Inf. Service, Springfield, VA 22161.

${ }^{15}$ B. I. Cohen, B. F. Lasinski, A. B. Langdon, and E. A. Williams, Phys. Plasmas 4, 956 (1997).

${ }^{16}$ A. V. Maximov, W. Rosmus, V. T. Tikhonchuk, D. F. Dubois, H. A. Rose, and A. M. Rubenchik, Phys. Plasmas 3, 1689 (1996).

${ }^{17}$ J. A. Heikkinen, S. J. Kartunen, and R. R. E. Salomaa, Phys. Fluids 27, 707 (1984).

${ }^{18}$ V. T. Tikhonchuk, C. Labaune, and H. A. Baldis, Phys. Plasmas 3, 3777 (1996).

${ }^{19}$ D. F. Dubois, B. Bezzerides, and H. A. Rose, Phys. Fluids B 4, 241 (1992). 


\title{
Effect of crossed beams irradiation on parametric instabilities
}

\author{
C. Labaune1, H.A. Baldis' ${ }^{2}$, B.S. Bauer ${ }^{1 *}$, E. Schifano1, A. \\ Michard 1 \\ ${ }^{1}$ Laboratoire pour l'Utilisation des Lasers Intenses, Ecole Polytechnique, \\ Centre National de la Recherche Scientifique, 91128 Palaiseau cedex, \\ France \\ 2Institute for Laser Science and Applications (ILSA) \\ Lawrence Livermore National Laboratory, POB 808, Livermore CA 94550,
} USA

\begin{abstract}
Modification of the growth of scattering processes in the case of multiple beam irradiation compared to single beam. irradiation has been investigated in a preformed plasma using Thomson scattrering of a short wavelength probe beam, and spectral and temporal analysis of reflected and transmitted light. First observations of the reduction of the amplitude of ion acoustic waves associated with stimulated Brillouin scattering, amplification of the amplitude of electron plasma waves associated with stimulated Raman scattering, and transfer of energy between crossing beams with same frequency in a flowing plasma under crossed beam irradiation are reported.
\end{abstract}

\section{Introduction}

In the indirect drive approach to inertial confinement fusion (ICF) several laser beams will be focused and overlap near the laser entrance hole. Interaction processes between these beams and the underdense plasma in this region can be influenced by crossing effects. Parametric instabilities (Kruer 1988 ; Baldis et al. 1991), like stimulated Brillouin and Raman scattering (SBS and SRS) and filamentation, have deleterious effects for laser fusion (Lindl 1995) and the modification of 
their growth under multiple beam irradiation compared to single beam irradiation must be understood to design efficient fusion scheme. Energy transfer between crossing beams can affect the initial symmetry which is controlled by adjusting the relative power of the different beams.

Modification of interaction processes between one laser beam and a plasma, in the presence of other laser beams, can proceed from different effects. The region of beam overlap will result in higher intensity than was present in an individual beam, and non linear processes will be affected by this increase of intensity. Theoretical studies have predicted that collective parametric instabilities can have lower thresholds than single-beam instabilities when considering many overlapping beams (DuBois et al.1992 ; Rose et al. 1992). In the cases of SBS and SRS the geometry and the symmetry of the beams are important to produce this effect. A well-defined resonance of the amplitude of ion acoustic waves associated with SBS has been observed along the bisecting direction between two laser beams (Baldis et al. 1996). In the case of two beams, energy transfer through ion acoustic waves has been studied both in the case of beams with different frequencies in homogeneous plasmas, and in the case of beams with same frequency in presence of plasma flow (Kruer et al. 1996 ; Eliseev et al. 1996 ; McKinstrie et al. 1996). Energy transfer between two frequencymismatched laser beams has been observed to be maximum when the difference in the frequencies of the two beams was comparable to the frequency of the ion acoustic wave (Kirkwood et al. 1996). It has also been observed that crossing $\mathrm{CO}_{2}$ laser beams of identical frequency can causes oscillations in the beam intensities on the time scale of the ion wave frequency by driving ion waves nonresonantly (Marsh et al.1994).

In this paper, we present experimental results on the modification of stimulated Brillouin and Raman growths in backward and side directions when two beams are simultaneously focused into the plasma compared to one beam irradiation. These results are based both on direct measurements of the amplitude of the plasma waves associated with these instabilities using Thomson scattering of a short wavelength probe beam, and on SBS reflectivities. We also report on the observation of energy transfer between two crossing beams with same frequency in a flowing plasma. These two experiments were performed with the same beam configuration and timing, but with different electron density of the plasma. In the following, we will refer to experiment 1 for the SBS, SRS studies, and experiment 2 for the transfer of energy study. 


\section{Experimental set-up}

The experiments have been performed using the six-beam laser facility at LULI, to produce the interaction between one or two $1.053 \mathrm{~mm}$ laser pumps and a well-characterised underdense, preformed plasma. A scheme of the laser beam configuration and timing is shown in Figure 1.

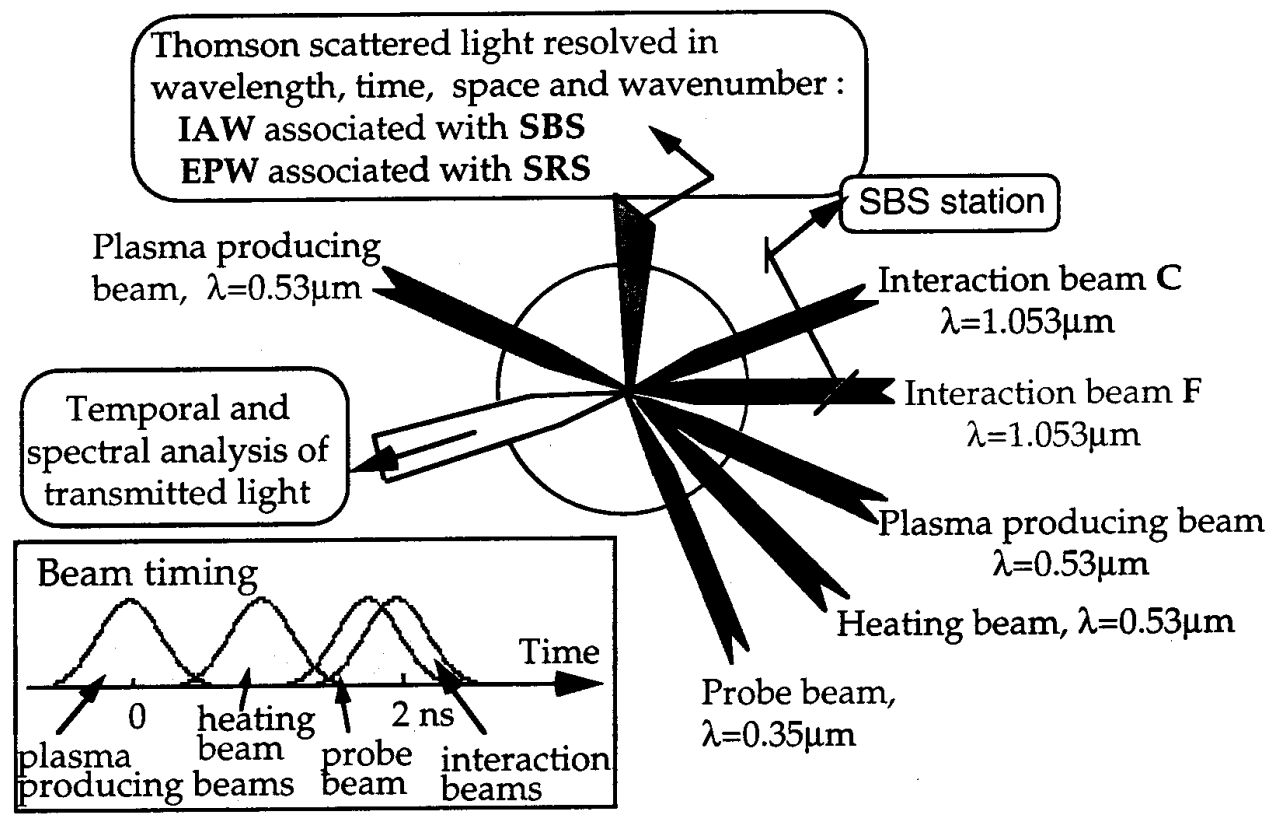

FIGURE $1:$ Scheme of the experimental set-up

Three $0.53 \mu \mathrm{m}$ beams were used to preform and heat the plasma from a thin exploded foil. Two identical $1.053 \mu \mathrm{m}$ laser beams were focused with $\mathrm{f} / 6$ lenses through random phase plates (RPP) in the plasma with 2 ns time delay with respect to the plasma forming beams. Their focal spot diameters were $320 \mu \mathrm{m}$, and maximum average intensities were $10^{14} \mathrm{~W} / \mathrm{cm}^{2}$. The angle between the two beams was $22.5^{\circ}$ and the axis of symmetry of the plasma was placed on the main interaction beam axis which were beams $F$ and $C$, respectively for experiments 1 and 2. For some shots the heating beam was used as an interaction beam to study the effect of the geometry of the crossing 
beams with respect to the Thomson diagnostic. The sixth beam was used as a probe beam, with $0.35 \mu \mathrm{m}$ wavelength, to produce scattering off the plasma waves produced by the parametric instabilities.

The targets were thin plastic $(\mathrm{CH})$ foils, with initial foil thickness $1.2 \mu \mathrm{m}$. The electron density of the plasma was modified by changing the total energy of the three plasma producing beams from $150 \mathrm{~J}$ to $15 \mathrm{~J}$ (at $2 \omega$ ). In the first case (experiment 1), the plasma was fully characterised using time-resolved spectra of the Thomson scattered light off electron plasma waves associated with SRS. Temporal evolution of the electron density profiles along the laser axis and temperature were deduced from these spectra during the interaction laser pulse. The maximum electron density, on the laser axis, varied between 0.3 and $0.08 n_{c}$ (where $n_{c}$ is the critical density for $1.053 \mu \mathrm{m}$ light), the density scalelength was 500 $\mu \mathrm{m}$, and the electron temperature was $0.5 \mathrm{keV}$. Comparison with 2D hydrodynamical simulations provided good agreement with these measurements. The second type of plasma (experiment 2) was characterised using spatial and temporal evolution of the $5 / 2 \omega$ emission, produced by the coupling between the $3 \omega$ probe beam and $\omega / 2$ plasmon produced by the two plasmon decay of the interaction beam. This harmonic emission provided information on the position of the quartercritical layer as a function of time and comparison with 2D hydrodynamical simulations indicated that the electron density on the target axis evolved from 0.45 to 0.2 nc during the interaction pulse.

The geometry of the Thomson scattering diagnostic was designed so the collected light corresponded to backscatter instabilities of beam F, both for SBS and SRS. The scattered light was sent to three streak cameras, of which two were coupled with spectrometers. The temporal, spectral, and spatial intensity of electron plasma waves (EPW) and of ion acoustic waves (IAW) associated respectively with SRS and SBS was analysed. The second important diagnostic for these experiments was another spectrometer coupled with a streak camera which provided time-resolved spectra of the SBS light collected in the focusing lens of beam $F$ in experiment 1 , and of light transmitted along beam $C$ in experiment 2 . 


\section{Effect of crossing beams on SBS and SRS in backward and side directions}

We have studied the effect of crossing beams on SBS and SRS by measuring the variation of the scattered light intensity off the plasma waves as a function of the second beam intensity. Intensity of the Thomson scattered light is proportional to the square of the plasma waves amplitude, so it gives direct information on the growth of the scattering mechanisms. Results are shown in Figure 2. They represent the total scattered intensity, integrated in space, time and spectrum off IAW associated with SBS of beam F (Fig. 2-a), and off EPW associated with SRS of beam F (Fig. 2-b). In both cases we have observed that the scattered intensity off plasma waves associated with beam $\mathrm{C}$ was negligible compared to the scattered intensity off plasma waves associated with beam $F$. This is in agreement with what was expected from the amplitude of plasma waves associated with sidescattering processes compared to backscattering processes.
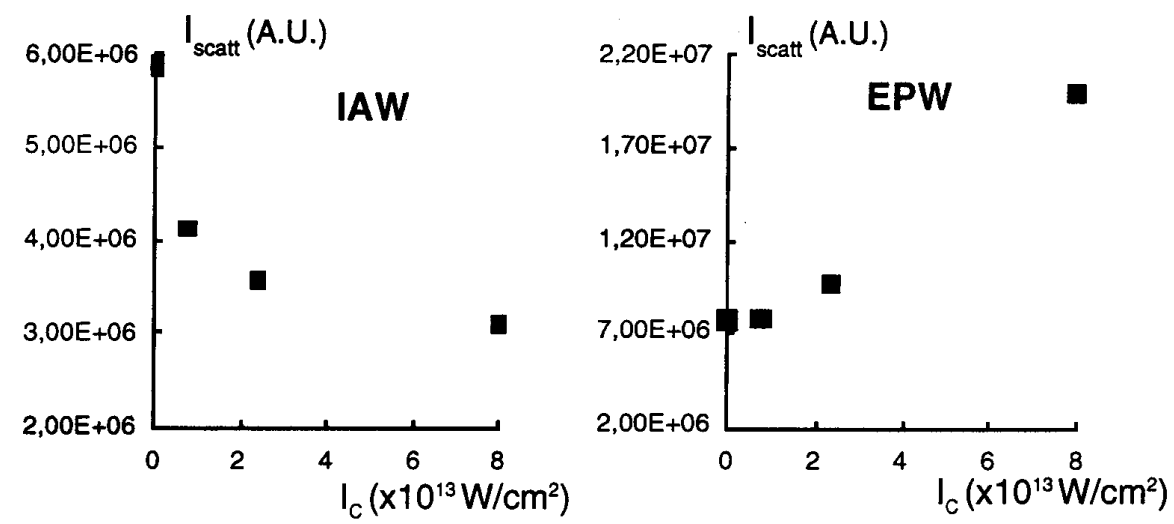

FIGURE 2 : Modification of the intensity of the Thomson scattered light off ion acoustic waves (IAW) associated with stimulated Brillouin scattering and electron plasma waves (EPW) associated with stimulated Raman scattering as a function of the secondary beam intensity

These results are the first observation of the reduction of the amplitude of ion acoustic waves associated with stimulated Brillouin scattering and amplification of the amplitude of electron plasma waves associated with stimulated Raman scattering under crossed beam 
irradiation. A reduction of the SBS light collected in the focusing lens of beam $F$ was observed in the case of two beams irradiation in agreement with the reduction of the IAW intensity.

More complete studies indicate that same effects happen with different geometries of the beams with respect to each other and with respect to the Thomson scattering diagnostic, but that the reduction or amplification factors depend on the geometry. This indicates that the observed effects are not produced simply by the increase of intensity, or modification of the intensity distribution, in case of two beams irradiation. We have also observed independent modification of the amplitude of IAW and EPW when using different geometries, which indicates that the observed results can not be explained only by the competition between SBS and SRS.

We think that coupling between the plasma waves associated with the two beams is the most likely process to produce the observed change of growth of SBS and SRS. In the case of SBS, it has been observed in numerical simulations (Cohen et al. 1995), that the presence of secondary IAW produced by another beam can decrease the growth of the primary IAW because of mode coupling. The secondary IAW are acting as a perturbation to the SBS growth, and total SBS is reduced in case of multiple beam irradiation compared to one beam irradiation. This was an unexpected result, which is a good news for ICF experiments. This interpretation is in agreement with previous experiments which showed a very narrow resonance on the amplification of IAW amplitude for IAW along the bisecting direction between the two beams, and reduction of IAW amplitude for all the other directions. In the case of experiments described in this paper, the scattered light was collected in an angle three times larger than the one used to observe the resonance effect, so that the reduction is stronger than the amplification which can not be observed. The amplification of EPW associated with SRS is not well understood yet. It may be produced by the seeding of the instability by the other beam through resonance conditions between the electron plasma waves. These resonance conditions may be easier to get than for the IAW, because of the dependency of the EPW frequency on electron density. This interpretation is in agreement with the fact that the amplification factor depends on the position of the beams with respect to the Thomson scattering diagnostic. 


\section{Transfer of energy between crossing beams in a flowing plasma}

The transmitted energy of beam $C$ was collected with an $f / 3$ lens, off-axis from beam $\mathrm{C}$ by $3^{\circ}$, and with a larger aperture than the one of beam $C$. This light was temporally and spectrally resolved. We studied the total level and spectral composition of transmitted energy of beam $C$ with and without beam F. Figure 3 shows the temporal evolution of the transmitted energy in the two cases : beam $\mathrm{C}$ alone, and beams $\mathrm{C}+\mathrm{F}$. We observe an overall increase of the total transmitted energy by a factor 2.3 in presence of beam $F$. To understand this result, we spectrally resolved the transmitted light. Figure 4 shows the time-resolved spectra of the transmitted light for the two cases : beam $\mathrm{C}$ alone, and beams $\mathrm{C}+\mathrm{F}$. In both cases, we observe two spectral components. The first one is unshifted from the initial laser frequency, starts early during the interaction pulse and stops before the end. Its spectral width is the same as the one of transmitted energy through vacuum and is defined by the spectrometer. The second one is red-shifted by a few Angström, is broad, with temporal and spectral structures, starts later than the first component, and lasts until the end of the laser pulse.

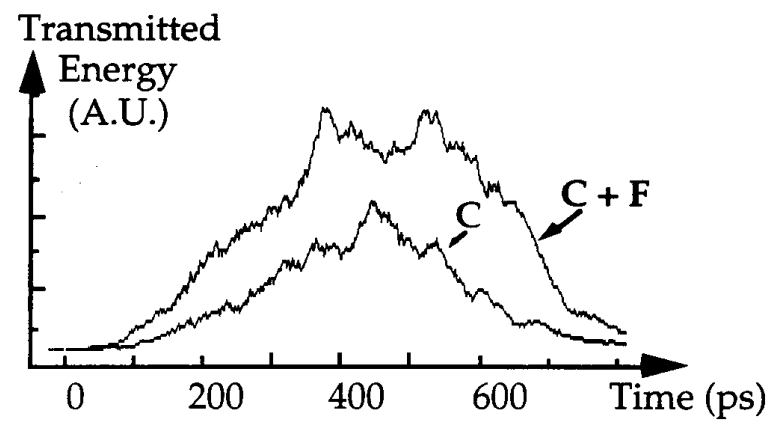

FIGURE 3 : Transmitted energy along beam $C$ direction for the two cases : beam $C$ alone, and beam $\mathrm{C}$ in the presence of beam $\mathrm{F}$. We observe an amplification of the transmitted energy by a factor 2.3 in the case of two beams irradiation.

The unshifted component is no much modified by the presence of beam $F$ as can be seen by comparing Fig. 4-a and $b$, while the red-shifted component is strongly enhanced by the presence of beam $F$. The 
amplification factor (total transmitted energy with beams $\mathrm{C}+\mathrm{F}$, divided by total transmitted energy with beam $\mathrm{C}$ alone) is 0.9 for the unshifted component and 3.5 for the red-shifted component. So, the amplification of beam $\mathrm{C}$ is mainly due to the amplification of the red-shifted component. We have also observed that the intensity of the red-shifted component depends on the energy of beams $C$ and $F$, so is produced by a non linear effect, and is in the non-saturated regime.

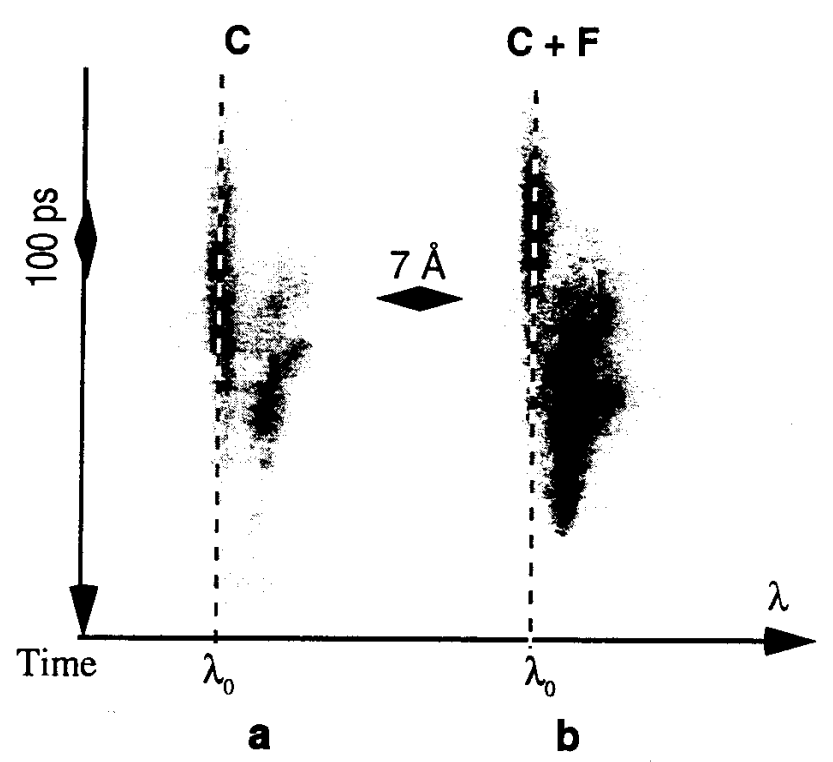

FIGURE 4 : Time-resolved spectra of the transmitted light along beam $\mathrm{C}$ for the two cases : beam $\mathrm{C}$ alone, and beam $\mathrm{C}$ in the presence of beam $\mathrm{F}$.

The measured spectral shift of the red-shifted component is much larger than what could be expected from forward stimulated Brillouin in this plasma. This one is related to the scattering angle $\theta$, to the elctron density relative to critical density and to the electron temperature $T_{e}$ :

$$
\delta \lambda_{(\AA)}=15.9\left(1-\mathrm{n}_{\mathrm{e}} / \mathrm{n}_{\mathrm{c}}\right)^{1 / 2} \sin (\theta / 2) \mathrm{T}_{\mathrm{e}(\mathrm{keV})}^{1 / 2}
$$

For our conditions, $\theta \sim 10^{\circ}$, Te $\sim 0.5 \mathrm{keV}, \mathrm{n}_{\mathrm{e}} / \mathrm{n}_{\mathrm{c}} \sim 0.2, \delta \lambda$ is of the order of $1 \AA$. Self-phase modulation of the laser light in a rapidly varying electron density plasma could produce this type of shift (Yamanaka et al. 1975 ; Labaune et al. 1985 ; Afshar-Rad et al. 1991). This effect produces a frequency shift : $\delta \omega=\frac{\omega}{c} \frac{d}{d t} \int\left(1-n_{e} / n_{c}\right)^{1 / 2} d z$ and a wavelength shift 
$\delta \lambda=-\frac{\lambda \delta \mathrm{z}}{\mathrm{c}}\left(1-\mathrm{n}_{\mathrm{e}} / \mathrm{n}_{\mathrm{c}}\right)^{-1 / 2} \frac{\mathrm{d}\left(\mathrm{n}_{\mathrm{e}} / \mathrm{n}_{\mathrm{c}}\right)}{\mathrm{dt}}$ after a propagation of $\delta \mathrm{z}$. The overall evolution of the electron density due to plasma expansion is not fast enough to produce the observed frequency shift. Filamentation of the laser light in the active speckles could strongly enhance self-phase modulation because of the rapid decrease of electron density in the filaments and produce shifts of a few Angstroem, as observed in the experiment. This phenomena is also in agreement with the decrease of the shift as a function of time, going to zero at the end of the pulse, when $d\left(n_{\mathrm{e}} / \mathrm{n}_{\mathrm{c}}\right) / d$ goes to zero. Because of the non linear behaviour of the enhancement of the transmitted light in presence of the secondary beam, our observations could be interpreted as due to enhancement of forward stimulated Brillouin scattering in self-focused speckles.

\section{Conclusion}

The experimental results presented in this paper are the first evidence that overlapping beams can have a detrimental effect on the growth of backward SBS. Long wavelength ion fluctuations associated with sidescattering of the other beams could produce a non-linear inhibition of SBS by introducing an additional damping. On the other hand, overlapping beams can enhance the growth of backward SRS, and modify the transmitted light of one beam. This latter may be due to enhanced forward SBS in filaments and can affect the initial intensity balance between the beams in a fusion experiment. Experiments with crossing beams can allow well-controlled studies of parametric instabilities and they should allow to better understand many aspects of the growth of SBS and SRS, including their competition.

\section{Acknowledgment}

The authors gratefully acknowledge the support of the technical groups of LULI during the experiment. This work was partially supported under the auspices of the U.S. Department of Energy by the Lawrence Livermore National Laboratory under contract No.W-7405ENG-48. Part of this support was provided through the LLNL-LDRD program under the Institute for Laser Science and Applications. 


\section{REFERENCES}

* Present address Department of Physics, University of Nevada, Reno, USA

KRUER W. L., The Physics of Laser plasma Interactions (Addison Wesley Publishing Co., Redwood City, CA, 1988).

BALDIS H.A, E.M. CAMPBELL, AND W.L. KRUER, "Laser-plasma interactions", in Physics of Laser Plasmas, Handbook of Plasma Physics, North-Holland, Amsterdam 1991, p.361-434.

LINDL W. L. , Phys. Plasmas 2, 3933 (1995).

DUBOIS D.F., B. BEZZERIDES, and H.A. ROSE, Phys. Fluids B4, 241 (1992) ; H.A. ROSE, and D.F. DUBOIS, Phys. Fluids B4, 252 (1992).

BALDIS H.A., C. LABAUNE, E, SCHIFANO, N. RENARD, A. MICHARD, Phys. Rev. Lett. 77, 2957 (1996).

KRUER W. L., S.C. WILKS, B.B. AFEYAN, andR.K. KIRKWOOD, Phys. Plasmas 3, 382 (1996).

ELISEEV V.V., W. ROZMUS, V.T. TIKHONCHUK, C.E. CAPJACK, Phys. Plasmas 3, 2215 (1996).

MCKINSTRIE C.J., J.S. LI, R.E. GIACONE, and H.X. VU, Phys. Plasmas 3, 2686 (1996).

KIRKWOOD R.K. et al., Phys. Rev. Lett. 76, 2065 (1996).

MARSH, K., C. JOSHI, AND C. MCKINSTRIE, Bull. Am. Phys. Soc. 39, 1585 (1994).

COHEN B.I., B.F. LASINSKI, A.B. LANGDON, E.A. WILLIAMS, H.A. BALDIS, K.G.

ESTABROOK, R. KIRKWOOD, K. WHARTON andC. LABAUNE, Bull. Am. Phys. Soc. 42, 10, 1965 (1997).

YAMANAKE C. et al., Phys. Rev. A11, 2138 (1975).

LABAUNE C. et al., Phys. Rev. A 32, 577 (1985).

AFSHAR-RAD et al., Euro Phys. Lett. 15, 745 (1991).7 